\title{
The TFOS International Workshop on Contact Lens Discomfort: Report of the Contact Lens Materials, Design, and Care Subcommittee
}

\author{
Lyndon Jones, ${ }^{1}$ Noel A. Brennan, ${ }^{2}$ José González-Méijome, ${ }^{3}$ John Lally, ${ }^{4}$ \\ Carole Maldonado-Codina, ${ }^{5}$ Tannin A. Schmidt,${ }^{6}$ Lakshman Subbaraman, ${ }^{1}$ Graeme Young, ${ }^{7}$ \\ Jason J. Nichols, ${ }^{8}$ and the members of the TFOS International Workshop on Contact Lens \\ Discomfort
}

${ }^{1}$ Centre for Contact Lens Research, School of Optometry and Vision Science, University of Waterloo, Waterloo, Ontario, Canada ${ }^{2}$ Johnson \& Johnson Vision Care, Inc., Jacksonville, Florida

${ }^{3}$ University of Minho, CEORLab, Center of Physics (Optometry), Gualtar, Braga, Portugal

${ }^{4}$ C. R. Bard, Inc., Bard Medical Division, Covington, Georgia

${ }^{5}$ The University of Manchester, Faculty of Life Sciences, Manchester, United Kingdom

${ }^{6}$ University of Calgary, Faculty of Kinesiology, Calgary, Alberta, Canada

${ }^{7}$ Visioncare Research Ltd., Farnham, Surrey, United Kingdom

${ }^{8}$ The Ocular Surface Institute, University of Houston College of Optometry, Houston, Texas

Correspondence: Jason J. Nichols, The Ocular Surface Institute, University of Houston College of Optometry, 505 J. Davis Armistead Building, 4901 Calhoun Road, Houston, TX 77204-2020;

jnichols@optometry.uh.edu.

See tables in the Introduction for the members of the TFOS International Workshop on Contact Lens Discomfort.

Submitted: September 6, 2013

Accepted: September 6, 2013

Citation: Jones L, Brennan NA, González-Méijome J, et al. The TFOS International Workshop on Contact Lens Discomfort: Report of the Contact Lens Materials, Design, and Care Subcommittee. Invest Ophthalmol Vis Sci. 2013;54:TFOS37-TFOS70. DOI:10.1167/iovs.13-13215

Keywords: contact lens, materials, polymers, dry eyes, designs

\section{Introduction and Goals of the Materials, Design, and Care Subcommittee}

Wamining the role of the contact lens material, design, and the care system is fundamental to understanding contact lens discomfort (CLD). However, a systematic review that tries to determine the governing factors is fraught with difficulties. A lack of a validated "instrument" (or single validated questionnaire) for measuring discomfort makes it impossible to compare between studies because reported levels of comfort (or discomfort) are inconsistent. Subject classifications can vary widely, from studies that include only neophytes or asymptomatic contact lens (CL) wearers to studies including only those contact lens-wearing subjects who experience marked dryness or symptoms of discomfort. Also, it is difficult to measure issues of importance in isolation because changing one factor in a contact lens or care solution can invariably affect another. An illustration of this relates to a change in hydrogel water content, which also affects oxygen permeability, oxygen transmissibility, modulus, and possibly lens thickness. Finally, various confounding factors between studies also make true comparisons problematic. Typical examples would include differences between brands of lenses made from the same material (which may have differing geometric designs, edge configuration, or production methods); wearing modality (lenses may be worn on a daily wear [DW] basis, overnight occasionally, or for up to
30 nights on a continuous wear [CW] basis); duration of use prior to replacement, wearing time during the day (from just a few hours to most of the day); and care product differences or exposures (which could range from no exposure in the case of daily disposable [DD] materials to a preserved system that has extensive uptake and release from the contact lens material being examined).

The purpose of this report is to summarize evidence-linking associations, mechanistic and etiological factors between contact lens materials, designs, and care solutions with CLD. The potential factors associated with this are many and varied, and graphically display the complexity of this issue.

\section{Contact Lens Materials}

Given the fact that approximately $90 \%$ of the world's contact lens wearers are wearing soft lenses with no recent change in this figure, ${ }^{1}$ this report primarily concerns itself with the role of soft lens materials and designs and care solutions in CLD, with some discussion of rigid gas permeable lens (RGP) materials or designs where appropriate.

\section{Conventional Hydrogel Materials}

The pioneering work of Wichterle and colleagues ${ }^{2,3}$ is well known as a basis for the development of hydrogel polymers for soft contact lenses, including lightly cross-linked polymers of 2- 
hydroxyethyl methacrylate (polyHEMA, 38\% equilibrium water content [EWC]). Subsequent versions of polyHEMA-based materials with increased EWCs were made by copolymerizing it with both hydrophobic monomers (e.g., methyl methacrylate [MMA]) or other monomers of varying hydrophilicities (e.g., $N$-vinyl pyrrolidone [NVP]; methacrylic acid [MAA]). ${ }^{4}$ Appendix A provides an overview of the characteristics of some commonly prescribed hydrogel materials. It was believed that a higher EWC would lead to a more wettable and comfortable lens (and increased oxygen transmissibility). However, it soon became apparent that dehydration is more pronounced with higher water hydrogels, particularly those with higher amounts of free water., ${ }^{5,6}$ This was sometimes associated with corneal desiccation staining ${ }^{5,7}$ and ultimately reduced end-of-day comfort. ${ }^{8}$

In an attempt to enhance the biocompatibility of soft lenses, a novel material combining polyHEMA with a synthetic analogue of phosphorylcholine (PC) with water content of approximately $60 \%$ (omafilcon A) was developed in the early 1990s. ${ }^{9}$ The introduction of hioxifilcon A in the late 1990s, where a nonionic copolymer of polyHEMA and glycerol methacrylate (GMA) were combined, was claimed to achieve excellent biomimicry by imitating the wetting properties of mucin. These approaches were designed in part to resist oneye dehydration and deposition, although improvements in comfort were varied. ${ }^{10-18}$

\section{Silicone Hydrogel Materials}

Despite many attempts to harness the oxygen permeability of silicone rubber in contact lens materials, it was not until the late 1990s that two low-water content silicone hydrogel (SiHy) contact materials, lotrafilcon A (24\% water) and balafilcon A (36\% water), were released. The original intent for silicone hydrogels (due to their very high oxygen permeability) was for use as extended wear (EW) materials, ${ }^{19}$ but their use for daily wear has since become dominant (including their use as daily disposables). ${ }^{1}$ Silicone hydrogel development typically focused on compositions or macromers based on silicone-containing monomers (TRIS, siloxy macromer) that are sufficiently compatible with a range of hydrophilic monomers (including $N, N$-dimethyl acrylamide, NVP, polyHEMA) ${ }^{4,20-29}$ Although the siloxane groups confer high oxygen permeability, they also give rise to inherent wettability issues, so several strategies have been employed to render SiHy surfaces more hydrophilic. Appendix B provides an overview of the characteristics of some SiHy materials.

\section{Bulk Properties of Soft Lens Materials}

Water Content and Ionicity. Equilibrium water content and ionicity are used to classify lens materials by the Food and Drug Administration (FDA) and International Organization for Standardization, because of their impact on clinical performance. ${ }^{30,31}$ Although the relevance of such a grouping has been confirmed for such factors as dehydration and deposition, the relation with wearing comfort is less clear.

Nichols and Sinnott ${ }^{8}$ reported higher odds (odds ratio [OR]: 2.25) for CL-related dry eye in patients wearing high EWC lenses, but ionicity was not related with dryness symptoms. In a follow-up analysis, Nichols and colleagues ${ }^{32}$ showed that when compared with FDA Group I materials (the referent material), both FDA Groups II and IV were associated with a 2 to 3 times increased odds of contact lens dry eye. Further, in a small study with 10 subjects, Wilson and colleagues ${ }^{33}$ reported better comfort for patients fitted randomly with an FDA Group II lens (nelfilcon A, non-ionic high EWC) compared with an FDA Group IV (etafilcon A, ionic high EWC) lens. Similar results were found by Guillon and colleagues ${ }^{34}$ when comparing the same materials in 22 patients in a crossover study wearing the lenses for 1 week in a random order. However, these studies ignore the effect of lens design, and the differences cannot be exclusively attributed to the different material properties.

Efron and colleagues compared the initial comfort of low (38\%), medium (55\%), and high (70\%) EWC lenses and concluded that lower water content materials were more comfortable than higher EWC lenses in a nondispensing study where comfort was rated after 5 minutes of wear. ${ }^{35}$ This study excluded potential confounding factors such as edge design or surface finishing, as all lenses were lathe-cut in an identical design by the same manufacturer. Young ${ }^{36}$ also evaluated comfort in a study aiming to predict the success of fitting low (38\%), medium (54\%-58\%), and high EWC (69\%-74\%) contact lenses. The results suggested improved comfort for low EWC lenses: First, the average comfort score was higher ( 8.4 for low, 8.2 for medium, and 8.2 for high EWC lenses). Second, low EWC flat-fitting lenses were significantly more comfortable than medium and high EWC lenses. ${ }^{36}$

Silicone hydrogel materials have an additional confounding factor to the understanding of the potential role of EWC on CLD. The majority of these lenses have a low EWC, but they have substantially differing oxygen transmissibilities, modulus values, and surface wetting properties from traditional hydrogel materials. Dumbleton and colleagues ${ }^{37}$ conducted a $^{2}$ study to evaluate the comfort of five different SiHy materials randomly fitted for 1-month periods, using a crossover design. All lenses generally performed similarly at the end of each period, although there was a slight difference for the ionic lens material to be associated with lower comfort at dispensing. Thus, the potential influence of material properties other than EWC or ionicity prevent any solid conclusions being drawn regarding the potential influence of these factors in SiHy material comfort.

In summary, several studies point to the increased comfort of low EWC lenses, with no direct impact of ionicity for conventional hydrogel materials. To date, no studies have been able to adequately draw any conclusions on the direct impact of these two factors for silicone hydrogels.

Oxygen Transmissibility. There has been a temptation to presume that oxygen transmissibility $(\mathrm{Dk} / \mathrm{t})$ is a key factor in contact lens comfort, and some of the circumstantial evidence and clinical dogma hints in this direction. Studies to determine the impact of oxygen transmissibility may be conducted either using lens materials of varying $\mathrm{Dk} / \mathrm{t}$ or using sealed goggles in which the oxygen tension is varied. Millodot found reduced corneal sensitivity after exposing the cornea to hypoxic gaseous environments for up to 10 hours, following shortterm wear of impermeable PMMA contact lenses and low Dk/t hydrogel lenses, and also cumulatively over years of wear of PMMA lenses. ${ }^{38-41}$ Contrary to the position that a greater supply of oxygen to the cornea might improve comfort, Millodot suggested that "a diminution of sensation with the wear of contact lenses is obviously beneficial as it helps the subject adapt more easily to the lenses." 42 To further this argument, the use of a topical anesthetic has been suggested as a means to assist adaptation to rigid contact lenses. ${ }^{43}$

Measurement of comfort while exposing the cornea to gaseous environments would be the obvious method of choice to discern the impact of hypoxia on comfort, but none of the studies in which this method has been employed has done so. It should be appreciated that many of the studies listed as evidence for or against an influence of $\mathrm{Dk} / \mathrm{t}$ on comfort were not necessarily designed with that specific purpose in mind (Table 1). Highlighting the shortfalls in such study designs to meet this end does not necessarily mean that they do not 
TABLE 1. Studies of Comfort With Lenses of Different Dk/t Values

\begin{tabular}{|c|c|c|c|c|c|c|c|c|c|c|c|c|}
\hline \multirow[b]{2}{*}{ First Author } & \multirow[b]{2}{*}{ Year } & \multicolumn{2}{|c|}{ Lenses } & \multirow[b]{2}{*}{ Modality } & \multirow{2}{*}{$\begin{array}{l}\text { Study } \\
\text { Type }\end{array}$} & \multirow[b]{2}{*}{$\boldsymbol{n}$} & \multirow[b]{2}{*}{ Sites } & \multirow[b]{2}{*}{ Duration } & \multirow[b]{2}{*}{ Rand } & \multirow[b]{2}{*}{ Masked } & \multirow{2}{*}{$\begin{array}{l}\text { Cont/ } \\
\text { Comp }\end{array}$} & \multirow{2}{*}{$\begin{array}{c}\text { Dk/t } \\
\text { Effect }^{*}\end{array}$} \\
\hline & & Test & Control & & & & & & & & & \\
\hline Brennan $^{44}$ & 2002 & SiHy & Hyd & $\mathrm{CW} / \mathrm{EW}$ & Pros & 212 & Multi & $1 \mathrm{y}$ & $\checkmark$ & & $\checkmark$ & $\checkmark$ \\
\hline Morgan $^{46}$ & 2002 & SiHy & SiHy & CW & Pros & 30 & Single & $2 \mathrm{mo}$ & $\checkmark$ & $\mathrm{S}$ & Xover & $\mathrm{N}$ \\
\hline Malet $^{45}$ & 2003 & SiHy & Hyd & CW/EW & Pros & 134 & Multi & $3 \mathrm{mo} / 1 \mathrm{mo}$ & $\checkmark$ & & Xover & $\checkmark$ \\
\hline Fonn $^{47}$ & 2003 & SiHy & Hyd & Open & Pros & 39 & Single & $7 \mathrm{~h}$ & $\checkmark$ & $\mathrm{I}, \mathrm{S}$ & Xover & $\mathbf{N}$ \\
\hline \multirow[t]{2}{*}{ Chalmers $^{48}$} & 2005 & SiHy & Hyd & $\mathrm{CW}$ and $\mathrm{EW} / \mathrm{EW}$ & Pros & 117 & Multi & $1 \mathrm{y} / \dagger$ & & & & $\checkmark$ \\
\hline & & SiHy & Hyd & $\mathrm{CW}$ and $\mathrm{EW} / \mathrm{DW}$ & Pros & 120 & Multi & $1 \mathrm{y} / \dagger$ & & & & $\checkmark$ \\
\hline Riley ${ }^{49}$ & 2006 & SiHy & Hyd & DW & Pros & 112 & Multi & $2 \mathrm{wk} / \dagger$ & & $\mathrm{S} \ddagger$ & & $\checkmark$ \\
\hline Santodomingo ${ }^{50}$ & 2006 & SiHy & SiHy & DW and EW & Pros & $21 / 24$ & Single & $1.5 \mathrm{y}$ & $\checkmark$ & & $\checkmark$ & $\mathbf{N}$ \\
\hline Dumbleton $^{51}$ & 2006 & SiHy & Hyd & DW & Pros & 87 & Single & $2 \mathrm{mo}$ & & $\mathrm{S} \neq$ & & $\checkmark$ \\
\hline Young $^{52}$ & 2007 & SiHy & Hyd & DW & Pros & 496 & Multi & $2 \mathrm{wk}$ & & & & $\checkmark$ \\
\hline Dillehay $^{53}$ & 2007 & $\mathrm{SiHy}$ & Hyd & DW & Pros & 779 & Multi & $2 \mathrm{wk}$ & & $\mathrm{S} \ddagger$ & & $\checkmark$ \\
\hline Cheung $^{54}$ & 2007 & SiHy & Hyd & DW & Pros & 30 & Single & $1 \mathrm{mo}$ & $\checkmark$ & $\mathrm{I}, \mathrm{S} \neq$ & $\checkmark$ & $\mathbf{N}$ \\
\hline Bergenske $^{55}$ & 2007 & SiHy & Hyd & EW/DW & Pros & 398 & Multi & $3 \mathrm{y} / \dagger$ & & & & $\checkmark$ \\
\hline Brennan $^{56}$ & 2007 & SiHy & SiHy & EW & Pros & 45 & Single & $1 \mathrm{y}$ & $\checkmark$ & & $\checkmark$ & $\mathrm{N}$ \\
\hline Schafer ${ }^{57}$ & 2007 & SiHy & Hyd & $\mathrm{CW}$ & Pros & 278 & Multi & $3 \mathrm{y} / \dagger$ & & & & $\checkmark$ \\
\hline Ousler $^{58}$ & 2008 & SiHy & Habit & DW & Pros & 11 & Single & $75 \mathrm{~min}$ & $\checkmark$ & $\mathrm{I}, \mathrm{S}$ & Xover & $\checkmark$ \\
\hline Dumbleton $^{37}$ & 2008 & SiHy & Hyd & DW & Pros & 55 & Single & $1 \mathrm{mo}$ & & & & $\checkmark$ \\
\hline Ozkan 59 & 2008 & SiHy & Hyd & DW & Pros & 15 & Single & $6 \mathrm{~h}$ & $\checkmark$ & $\mathrm{S}$ & $\checkmark$ & $\mathrm{X}$ \\
\hline Chalmers $^{60}$ & 2009 & SiHy & Hyd & t & Retro & $183 / 699$ & Multi & $\dagger$ & & & $\checkmark$ & $\checkmark$ \\
\hline Long $^{61}$ & 2009 & SiHy & Hyd & DW & Pros & 128 & Multi & $1 \mathrm{mo}$ & & & & $\checkmark$ \\
\hline Santodomingo ${ }^{62}$ & 2010 & SiHy & Hyd & $t$ & Pros & $24 / 22$ & Single & $3 \mathrm{~d}$ & & & $\checkmark$ & $\mathbf{N}$ \\
\hline Young $^{63}$ & 2011 & SiHy & Hyd & DW & Retro & $363 / 454$ & Multi & $\dagger$ & & & $\checkmark$ & $\checkmark$ \\
\hline Maissa $^{64}$ & 2012 & SiHy & Hyd & DW & Pros & 30 & Single & $10 \mathrm{~d}$ & $\checkmark$ & $\mathrm{I}, \mathrm{S} \neq$ & Xover & $\mathrm{X}$ \\
\hline Young $^{65}$ & 2012 & SiHy & Hyd & $t$ & Retro & $226 / 48$ & Multi & $\dagger$ & & & $\checkmark$ & $\mathbf{N}$ \\
\hline
\end{tabular}

Features of studies in which comfort between lenses with different oxygen transmissibility values can be at least in part compared. The primary purpose of the studies was not necessarily comparison of the lens types. All papers shown were published in peer-reviewed journals. Checkmark indicates feature is present. Slash mark is used to separate test and control group data where different. Cont/Comp, control or comparator lens run contemporaneously; Habit, habitual; Hyd, hydrogel; I, investigator masked; N, effect not shown; Pros, prospective; Rand, randomized study; Retro, retrospective; S, subject masked; SiHy, silicone-hydrogel; X, reverse effect shown; Xover, crossover study.

* Indicates whether lenses with higher Dk/t were more comfortable.

$\dagger$ Details not explicitly provided in the paper.

‡ Partially subject masked (assumes subjects would notice some differences in handling between SiHy and hydrogel lenses).

contain valuable information, but rather that using these studies to infer comfort is linked to oxygen supply is fraught with difficulties. For example, Brennan and colleagues ${ }^{44}$ and Malet and colleagues ${ }^{45}$ conducted open-label, multicenter, prospective, randomized studies with balafilcon A $(n=212)$ and lotrafilcon A $(n=134)$ SiHy lenses, respectively, worn in continuous wear (up to 29 nights without removal) with etafilcon A lenses as controls worn in extended wear (up to 6 nights). The Brennan study ${ }^{44}$ was contralateral eye and the Malet study ${ }^{45}$ was crossover. In both studies, the SiHy lenses were reported to perform better with respect to comfort than the control hydrogel lens. These are the only two peerreviewed studies that have used such designs and found these outcomes. However, both of these studies were open-label so the treatment arm was masked to neither subject nor investigator.

A multitude of studies have reported switching of existing hydrogel wearers out of their habitual lenses and into silicone hydrogels. ${ }^{37,48,49,51-53,55,57,61}$ Consistently, these studies have reported improvements in subjective response with the SiHy lenses compared with the hydrogels. However, the other common feature of these studies is the omission of a concurrent randomized, masked, control (e.g., "switching" back into a hydrogel lens) that would enable confirmation of a claimed improvement in comfort.

Six investigations that were randomized, partially controlled studies and were at least subject-masked have considered comfort differences between hydrogels and silicone hydrogels. Fonn and Dumbleton ${ }^{47}$ conducted a double-masked, contralat- eral, 7-hour, open-eye, nondispensing trial on 39 symptomatic and asymptomatic subjects. They found no difference between the hydrogel and SiHy lenses in comfort and dryness ratings. Cheung and colleagues ${ }^{54}$ conducted a prospective, doublemasked, contralateral eye study in which they compared the comfort of two weekly replacement $\mathrm{SiHy}$ and hydrogel lenses in 33 subjects over 1 month of daily wear. They were unable to detect a significant difference in subjective comfort scores between lens material types. In the only extended wear trial of this group, Martin and colleagues ${ }^{66}$ measured comfort after 7 days of contralateral eye contact lens wear of a SiHy and hydrogel in 20 subjects. They found that the SiHy lens was more comfortable and led to less dryness than the hydrogel lenses. In a single-center, double-masked, randomized, crossover, pilot clinical trial, Ousler and colleagues exposed 11 masked subjects to a controlled adverse environment for 75 minutes while wearing SiHy and habitual soft lenses. ${ }^{58}$ They found greater relief of subjective ocular discomfort associated with lens wear in adverse environmental conditions whilst wearing the SiHy. Ozkan and Papas, ${ }^{59}$ in a prospective, contralateral eye trial, compared comfort of a SiHy and hydrogel lens on 15 experienced lens wearers over 6 hours. Overall comfort was slightly (but significantly) higher for the low Dk hydrogel compared with the SiHy over this short time frame. Recently, Maissa and colleagues ${ }^{64}$ compared the comfort of four silicone hydrogels and one hydrogel in a prospective, crossover, double-masked, 10 day, daily wear trial. In rank order of comfort, the hydrogel was scored highest by 
the subjects and was statistically superior in comfort to one of the SiHy lenses at both the beginning and end of day.

The controls in each of these six prospective, randomized, subject-masked studies are inadequate to test whether $\mathrm{Dk} / \mathrm{t}$ alone is linked to lens comfort, as properties other than $\mathrm{Dk} / \mathrm{t}$ that may affect comfort, such as lens material surface properties and edge design, vary between the SiHy and hydrogel lenses under test. Nonetheless, the experimental designs are "more robust" than those other studies listed above, where subjects were swapped out of their habitual lenses to test lenses alone or where masking was inadequate. Interestingly, and in contrast to those studies, four of these better-executed studies did not find that SiHy lenses were superior in comfort to hydrogel lenses and indeed, in two of these studies, the hydrogel was more comfortable. Study design differences should be kept in mind when reconciling this apparent discrepancy. Overall, hydrogels seem to produce a more favorable comfort response in daily wear and shorterterm studies. During open eye wear, hydrogel lenses have sufficient $\mathrm{Dk} / \mathrm{t}$ to provide near normal oxygen supply, at least to the central cornea. ${ }^{67}$ The impact of the lower Dk/t of hydrogels will be exaggerated during eye closure, or extended or continuous wear. Importantly, the study by Martin and colleagues ${ }^{66}$ was under extended wear conditions and this may partially explain the difference in results between that and the other "more robust" designs. A further confounding factor is study duration. The longest time of follow-up for the randomized, subject-masked studies was 30 days, where the "inferior" study designs saw patients followed, in some cases, for 3 years.

In recent years, four large cross-sectional studies have compared comfort between SiHy and hydrogel lenses. As noted above, Ramamoorthy and colleagues ${ }^{32}$ presented detailed statistical analysis of a cross-sectional and nested case-control study of 360 participants. The authors found FDA material classification to be a strong predictor of contact lens-related dry eye classification. Silicone hydrogel lens wear was found to be significantly protective from dryness symptoms in a univariate regression, but dropped out in the final multivariate model with FDA Group. The authors suggested that this could be because silicone hydrogels are low in EWC and correlate with FDA grouping. This finding may be highly relevant in discussions on the influence of $\mathrm{Dk} / \mathrm{t}$ on comfort, as it points to the confounding effects of other material and lens properties. Chalmers and colleagues ${ }^{60}$ report on the analysis of a baseline self-administered questionnaire completed by 882 contact lens wearers comprised of 699 wearing hydrogel and $183 \mathrm{SiHy}$ lenses at 84 clinical sites. Diagnosis of dry eye increased with age in the hydrogel wearers from $10.6 \%$ (at $18-24$ years) to $21.1 \%$ (at $30-35$ years), while it remained steady at $19 \%$ in the SiHy wearers. While not precluding selection or survival bias, this study suggests that wearers of hydrogel materials find their lenses at least the equal of silicone hydrogels (with regard to symptoms of dryness) up to 30 years of age. Young and colleagues were involved in two prospective, multicenter, nonrandomized, cross-sectional, observational studies of soft contact lens-related dryness that were partially controlled for $\mathrm{Dk} / \mathrm{t}$ and where subjects responded to a questionnaire based on the Contact Lens Dry Eye Questionnaire (CLDEQ), from which they were classified as either having dry eye or not. In the first study of 932 daily wearers of soft contact lenses, the proportion of subjects scoring positive for contact lens dry eye did not differ by lens material (hydrogel versus SiHy). ${ }^{63}$ There were, however, differences in specific questions of typical and end-of-day comfort where the hydrogel performed less favorably. In their follow-up paper, they identified $226 \mathrm{soft}$ contact lens wearers with self-reported contact lens-related dryness as classified by a questionnaire and 48 asymptomatic control subjects. ${ }^{65}$ This study showed that participants with and without symptoms of CL-related dryness did not differ by SiHy lens use. Interpretation of these cross-sectional studies must be made in the context of aforementioned limitations, but overall, the studies provide somewhat equivocal results.

Collectively, the above studies cast doubt on the suggestion that the higher $\mathrm{Dk} / \mathrm{t}$ is associated with greater comfort. Therefore, it is not surprising that studies in which different brands of SiHy lenses are compared have been unable to discern a greater comfort response for those lenses with higher Dk/t. Morgan and Efron conducted a single-center, randomized, subject-masked, crossover study on 30 subjects wearing two brands of SiHy lenses each for 8 weeks. ${ }^{68}$ The brand with the higher Dk/t did not show superior comfort to the brand with the lower Dk/t. Santodomingo and colleagues considered the same two brands used in both daily wear and continuous wear in a total of 45 subjects for 18 months. ${ }^{50}$ Again, they found no superiority of comfort in the lens with higher Dk/t. Brennan and colleagues followed 45 subjects in a prospective, partially controlled randomized, partially masked contralateral eye study of three different SiHy lens brands for a period of 12 months. ${ }^{56}$ Again, the lens with the highest $\mathrm{Dk} / \mathrm{t}$ was not found to provide superior comfort.

While it may be appealing to attribute measured comfort benefits to the higher oxygen transmissibility of the SiHy lenses, a host of other confounding factors will drive the comfort response. Inadequacies of the control lens in experimental studies, including lens design, modulus, surface characteristics, and the modality of wear (daily disposable, reusable frequent replacement, flexible wear, extended wear, continuous wear) rather than $\mathrm{Dk} / \mathrm{t}$ per se, may be responsible for the outcomes achieved. Duration of wear, where adaptation to the higher modulus lenses takes place, might interact with the lens material effect. Other experimental biases in the form of selection, novelty, halo, Hawthorne, survival, or similar effects resulting from study designs that are inadequate to randomize or mask the control product may also influence the outcome.

In summary, there have been no Level I evidence studies that can provide an answer to the question of whether oxygen levels influence comfort. What can be said is the following:

1. Where lenses of higher $\mathrm{Dk} / \mathrm{t}$ are found to be more comfortable than lenses of lower $\mathrm{Dk} / \mathrm{t}$, there are deficiencies in experimental design or inadequacies in the control lenses that prevent definite attribution of such differences to oxygen.

2. There are circumstances where lenses of lower $\mathrm{Dk} / \mathrm{t}$ have been found to be more comfortable than lenses with higher Dk/t; therefore, any effect that oxygen may be having on comfort is being overshadowed by other factors or there simply may be no or a converse relation.

3. Where comfort differences between higher and lower $\mathrm{Dk} / \mathrm{t}$ lenses are found to be statistically insignificant, the method used to measure comfort may not be sufficiently sensitive to detect differences.

Modulus and Mechanical Factors. The two most important quantifiable mechanical properties are tear strength (elongation at break) and modulus, which can be measured in stretching (tensile or elastic) or compression (rigidity) mode. While modulus is a specific material parameter, the effective "stiffness" of a contact lens will also be influenced by its specific geometry (lens thickness profile) as a thick lens made from a low modulus material may still be considered relatively inflexible or stiff. A thinner lens made from a low modulus material will drape over the cornea, distributing itself evenly on the ocular surface with minimum lid interaction. In some 
instances, an increase in stiffness will help mask corneal astigmatism but possibly at the expense of initial comfort. ${ }^{69}$ Although the rigidity modulus has historically been useful for RGP materials, it is the tensile modulus that has primarily been most often quoted for soft lens materials. ${ }^{22,23,70-72}$

The first generation silicone hydrogels (lotrafilcon A, balafilcon $\mathrm{A}$ ) had tensile moduli that were significantly greater than most conventional hydrogels, ${ }^{22,23,71}$ such that for some wearers a comfort or wearer adaptation period was needed and there was an increased potential for mechanically induced ocular complications. ${ }^{19,21,73-75}$ Subsequent SiHy development has progressed to lower modulus materials through chemical structure modification and/or increased EWC. ${ }^{4,19,21-23,25,73,74}$

The higher modulus of SiHy materials was initially seen as an issue when refitting hydrogel wearers into these more oxygen permeable materials. However, when Riley and colleagues ${ }^{49}$ refitted 257 patients wearing hydrogel materials with a SiHy with a relatively low modulus (senofilcon A), they reported that $50 \%$ of subjects reported no contact lens-related discomfort. Most of the studies reporting refitting hydrogel wearers into silicone hydrogels report similar or higher levels of success, even when materials with a high modulus are employed. ${ }^{51}$ However, as noted recently by Guillon, ${ }^{76}$ the study design may partly explain these findings, as most refitting studies lack a concurrent control group or adequate masking. When comparing the ability of material to predict contact lens dry eye, Ramamoorthy and colleagues ${ }^{32}$ were unable to show any difference between the 11 (or more) individual materials being compared, including at least two SiHy materials. In the few studies reported where study bias was minimized by using a control group using low modulus hydrogel lenses, no differences in comfort between hydrogel and silicone hydrogels could be identified or attributed to modulus. ${ }^{47,54}$

Dehydration. Subjective reports of "dryness" and "discomfort" are well recognized as the main factors for contact lens discontinuation ${ }^{77,78}$; and this has remained unchanged over the last decade, regardless of the new lens materials introduced. ${ }^{79}$ This has led to an intuitive relationship being proposed between soft lens dehydration and discomfort, particularly at the end of the day. A connection between dehydration and discomfort seems plausible given: (1) the potential correlation between lens thickness and desiccation staining ${ }^{7,80}$; (2) the potential correlation between corneal staining and discomfort ${ }^{81,82}$; and (3) the increased friction presumably induced by dehydrated, dry lens surfaces. ${ }^{83}$ However, a proven relationship between dehydration and discomfort has been supported by relatively few studies. ${ }^{13,84,85}$ This is perhaps not surprising, given the difficulties in evaluating material dehydration and types of dehydration (e.g., initial temperature-induced dehydration followed by evaporative dehydration). ${ }^{86-88}$ It is this latter dehydration that is potentially problematic, as it produces a water gradient ${ }^{89,90}$ that draws water through the lens and, ultimately, results in corneal desiccation staining. ${ }^{7,91,92}$ Evaporative dehydration tends to be localized and therefore may result in only a small change in a given lens' overall water content. Likewise, evaporative dehydration may be less apparent with higher power lenses and, therefore, may be even more difficult to monitor in a subject group of varying prescription.

In addition to patient and environmental factors, differences in dehydration do exist between materials. A number of in vitro studies have shown that bulk water loss is closely related to initial EWC, with low EWC lenses (including silicone hydrogels) dehydrating less than higher EWC hydrogels. ${ }^{6,93-96}$ In studies that have evaluated lens dehydration $^{10,11,13,15,84,89,97-106}$ and also recorded comfort ratings, a significant relationship between the two has not been consistently shown
Hall and colleagues ${ }^{13}$ fitted four contact lens materials to 10 subjects and recorded dehydration and comfort after 4,8 , and 12 hours. At the 12-hour time-point there was a moderate negative correlation between comfort and dehydration for etafilcon A lenses $(r=-0.64, P=0.04)$, but no correlation for the remaining three materials. ${ }^{13}$ In a study in which omafilcon A was shown to dehydrate significantly less than other lenses of similar EWC (etafilcon A), Lemp and colleagues ${ }^{103}$ concluded from their 76-subject crossover study that the increased comfort found with the omafilcon A lenses was related to decreased on-eye dehydration.

In contrast with the work by Hall and Lemp, Fonn and colleagues $^{102}$ found no correlation, either in symptomatic ( $r=$ $0.33, P>0.05$ ) or asymptomatic subjects, between the change in lens water content for omafilcon $\mathrm{A}$ and etafilcon $\mathrm{A}$ and change in comfort over 7 hours of lens wear in a contralateral, double-masked, nondispensing study. Maldonado-Codina and Efron ${ }^{107}$ conducted a crossover study with 34 subjects to evaluate the impact of manufacturing technology and material composition on the clinical performance of five hydrogel lenses worn for 1 month each. Despite a significantly higher dehydration of the ionic (FDA Group IV) material after 6 hours and after 1 month of lens wear, there was no significant difference in overall comfort between lens types. Lastly, in perhaps the largest analyses of the relationship between material dehydration and comfort, Nichols and Sinnott ${ }^{8}$ and Ramamoorthy and colleagues ${ }^{106}$ showed that while indeed higher EWC hydrogel lenses tend to dehydrate to a greater degree than lower water lenses, the degree of dehydration was not associated with contact lens dry eye classification of the subjects.

In conclusion, considering the body of literature available, including several well-designed studies that attempted to address this topic, it is not likely that a causative or associative relation exists between on-eye bulk dehydration of materials and discomfort using the current methods used to capture either dehydration or subjective comfort.

\section{Surface Properties of Soft Lens Materials}

Friction and Lubricity. Lubrication, which can be defined as any means capable of controlling friction and wear of interacting surfaces in relative motion, provides defense against wear (the loss of material from interacting surfaces in relative motion usually related to friction). Materials with low friction and low wear are thought of as being well lubricated, or having good lubricity.

Friction coefficient measurements are most often made as an indicator of the quality of lubrication or lubricity, since wear measurements of biological surfaces are challenging. A friction coefficient is the ratio of the frictional force between two contacting surfaces in relative motion to the normal force between those surfaces. A variety of in vitro test setups with different test characteristics (scale, geometry, counter surface) and parameters (protocol, environment, lubricant, lens condition) have been used to assess friction coefficients of contact lenses. While each in vitro test setup has advantages and disadvantages, it remains unclear which, if any, is representative of in vivo function and/or friction and there are no standards on the techniques as such.

Several contact lens friction studies exist in the peerreviewed literature. ${ }^{83,108-115}$ Collectively, these studies demonstrate that friction associated with contact lenses is a challenging field of study, and support the notion that reported friction coefficients must always be considered in the context of the experimental parameters in which they were measured, which is outside the scope of this report. It is also important to note that while this is an expanding area of scientific interest, 


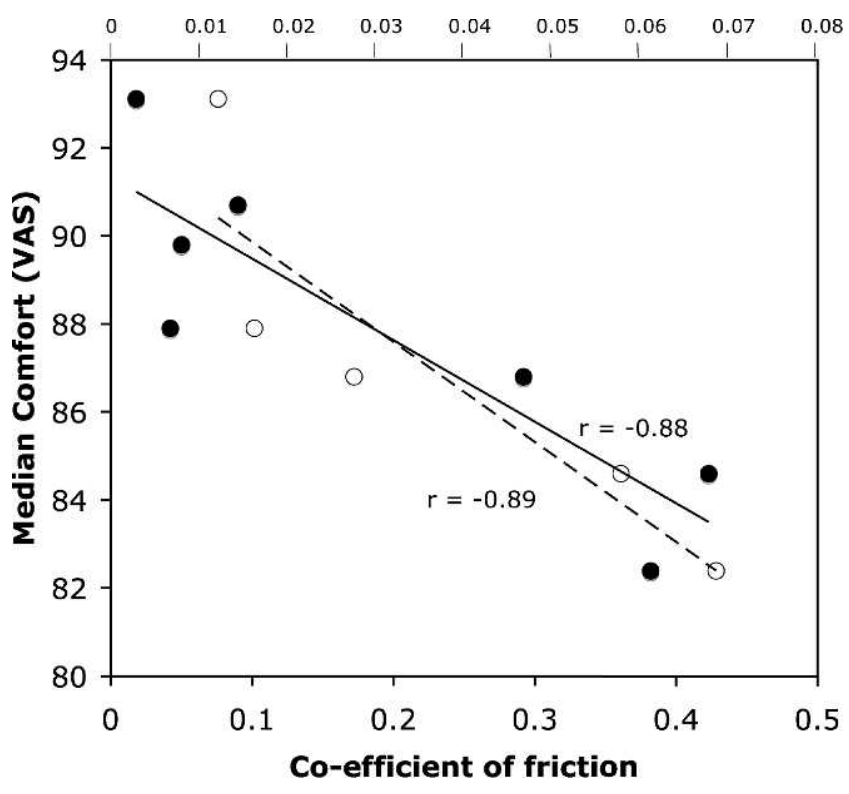

Figure. Plot of median end-of-day comfort from Brennan and Coles $^{116,117}$ versus coefficient of friction reported by Ross and colleagues $^{118}$ (open circles, dashed line, scale above plot area) and Roba and colleagues ${ }^{15}$ (closed circles, unbroken line, scale below plot area).

the aforementioned studies did not relate friction to comfort in contact lens wear.

Some recent evidence, albeit preliminary, does exist for an association between contact lens friction and comfort. ${ }^{116,117}$ Initially, coefficient of friction values from Ross and colleagues ${ }^{118}$ were compared with end-of-day comfort values from over 700 separate 1-month wearing trials and demonstrated a significant correlation $\left(r^{2}=0.79, P<0.01\right) .{ }^{116}$ More recently, peer-reviewed coefficient of friction data ${ }^{115}$ were used for the same analysis, and once again demonstrated significant correlations $\left(r^{2}>0.83 ; P<0.01\right) .{ }^{117}$ Additionally, these coefficient of friction data were shown to be highly correlated ( $r^{2}=0.91, P<0.0002$ ) with 2 -hour mean comfort data from work conducted by Andrasko investigating corneal staining ${ }^{119}$ (Figure).

Tucker and colleagues (Tucker R, et al. IOVS 2012;53:ARVO E-Abstract 6093) developed an inclined plane method to determine friction coefficients of a variety of soft contact lens materials. This data was then compared with subjective data for insertion comfort, overall comfort, and end-of-day comfort from a database of clinical trials in a more recent analysis (Kern J, et al. IOVS 2013;54:ARVO E-Abstract 494). A statistically significant relationship was demonstrated between lens coefficient of friction and subjective comfort, suggested to be clinically relevant given the range of friction coefficients measured, and an approximate 0.025 reduction in the friction coefficient (obtained from the inclined plane method) was associated with a 1-unit improvement in comfort on a 10-point scale.

Data from these two recent frictional studies (Kern J, et al. IOVS 2013;54:ARVO E-Abstract 494) ${ }^{117}$ provides the strongest evidence to date that contact lens lubricity may be associated with comfort, although again, there is no Level I evidence as such. A caveat on this interpretation is that the coefficient of friction and comfort data tend to cluster together by lens manufacturer; thus, a manufacturer might tend to have higher comfort scores and lower coefficient of friction for two or more lenses in their portfolio. It is conceivable that characteristics other than low coefficient of friction-for example, edge design-is common to the various lenses of that manufacturer and that one of these is the defining determinant of comfort.

Wettability. The term "wettability" is traditionally used to describe the tendency for a liquid to spread over a solid surface $^{120}$ and consequently has been widely adopted by the contact lens industry to describe the ability of the tear film to spread and remain on the surface of a contact lens. When a lens is applied to the eye, it fundamentally disrupts the normal tear film structure and physiology in a number of ways, including increasing the evaporation rate ${ }^{121-123}$ and decreasing tear film stability. ${ }^{121,124-126}$ The quality of the tear film over a lens is thought to play a key role in the lubrication of the lens/ ocular surface system and will ultimately influence how much friction and "wear and tear" will result.

Despite widespread use of the term, no physical measurement exists that can completely quantify wetting. Notwithstanding this limitation, a number of different laboratory (in vitro) and clinical (in vivo) techniques have been adopted to investigate the wetting properties of contact lens surfaces, details of which are outside the scope of this report. Wettability is thought to be important for all types of contact lenses, but in particular for silicone hydrogels, which tend to be more hydrophobic compared with their conventional hydrogel counterparts, at least in the laboratory.

In Vitro Wettability. In vitro investigations of wettability have provided us with a wealth of information about lens surfaces and what factors affect them in the laboratory. Overwhelmingly, reports in the literature document the investigation of soft lenses, particularly in recent years. Those that do investigate rigid lenses have shown that the contact angles obtained are significantly affected by the methodology $^{127,128}$ and since no recent reports exist that have used more current automated techniques, it is difficult to make any kind of meaningful comparisons with soft lenses. Studies have reported contact angles for unworn lenses in water or saline or other components ${ }^{129-134}$ and have shown that angles obtained for the same lenses can vary due to the differences in methodology or experimental conditions. ${ }^{135}$ Despite all of these data, none has been able to show any relationship between in vitro measurements and on-eye clinical wetting and, further, whether these laboratory measurements are in any way related to comfort. For example, both Nichols and colleagues $^{136}$ and Thai and colleagues ${ }^{134}$ investigated the effect of adding hydroxypropyl methylcellulose (HPMC) to a multipurpose contact lens solution and, despite differences in in vitro or in vivo wettability and tear film thickness, there was no overwhelming preference for either care solution.

Ex Vivo Wettability. In an attempt to make in vitro measures of contact angle more relevant, researchers have attempted to perform contact angle analysis on lenses postremoval, but there are surprisingly few publications that have measured the wettability of ex vivo contact lenses and related it to comfort. Tonge and colleagues ${ }^{137}$ measured dynamic contact angles of etafilcon A lenses after various periods of wear in lenses that had been presoaked in either saline or a surfactant; the surfactant-exposed lenses showed significantly lower-advancing contact angles than the saline-treated lenses (however, there was no statistically significant difference for the receding contact angle between the two treatments). Of particular note in the work was that comfort was reported as being better for the surfactant-soaked lenses compared with the saline-soaked lenses at all time points measured, although only six subjects were included. There appear to be no other studies that have related ex vivo wettability to comfort associated with contact lens wear.

In Vivo Wettability. In vivo wettability has been investigated using a range of relatively simple slit lamp-based procedures 
and grading scales, ${ }^{138,139}$ in addition to more indirect techniques such as the prelens noninvasive tear break-up time (NITBUT), ${ }^{102,140-143}$ tear thinning time, ${ }^{54,124,125,134,144}$ investigating the rate of evaporation from the lens surface, ${ }^{122,145}$ wavefront sensing, ${ }^{146}$ high-speed videokeratoscopy, ${ }^{147}$ and techniques based around specular reflection. ${ }^{148}$

One investigation compared comfort and NITBUT in nelfilcon A and nelfilcon A AquaRelease lenses. ${ }^{149}$ The authors reported that subjective ratings of comfort over a 16-hour period were consistently higher for the eye wearing the AquaRelease compared with the eye wearing the conventional nelfilcon A lens. NITBUT was greater with the AquaRelease than the conventional nelfilcon $\mathrm{A}$ lenses. In multivariate modeling, Nichols and Sinnott ${ }^{8}$ showed that prelens tear film thinning time was highly predictive of contact lens dry eye status, even when including EWC, osmolality, and lipid layer thickness (both significant themselves) in the multivariate statistical model.

Conclusive evidence that laboratory measures of contact angle can predict the wetting performance of a contact lens oneye is lacking. Furthermore, the link between clinical measures of wettability and contact lens comfort remains not understood, with some evidence that surrogate measures do show a relation. It is likely that the assessment of wettability provides us with an indirect method of investigating the lubrication present in the lens/eye "system" and conclusive results across numerous studies have eluded us because the techniques we have employed to probe the tear film do not accurately reflect its complex and dynamic nature. ${ }^{150}$

\section{Wetting Agent Incorporation}

The wetting agents discussed in this section of the review are limited to agents that are releasable and incorporated into contact lenses. Wetting agents in multipurpose solutions or contact lens packaging solutions will be addressed in another section. Wetting agents may be firmly embedded and provide enhanced wettability due to the materials being exposed at the lens interface or may be progressively released from the material over the course of the day.

Polyvinyl Alcohol. The nelfilcon A material is a polyvinyl alcohol (PVA)-based hydrogel specifically developed for use in a daily disposable lens. Maissa and colleagues ${ }^{151}$ suggested that the comfort level achieved with this lens "may result from a slow release of some residual entangled PVA" from the crosslinked PVA lens matrix. Using an in vitro release model, Tighe and colleagues ${ }^{152}$ suggested that the mechanical effect of the eyelid greatly accelerates soluble PVA release from the lens surface, which implies that the release is mechanically triggered or "blink activated" when placed on the eye. The next iteration of this material exploited this effect by intentionally adding nonfunctionalized PVA of appropriate molecular weight to enhance the elution of PVA, thereby increasing the comfort of these lenses. ${ }^{153}$ It was demonstrated in a contralateral eye study that adding this nonfunctionalized PVA enhanced tear stability and subjective comfort over a 16hour wearing period relative to the original nelfilcon A product. ${ }^{149}$ A further enhancement incorporated an optimized blend of nonfunctional PVA in the lens matrix coupled with hydroxypropyl methylcellulose (HPMC) and polyethylene glycol in the packaging saline. Tear film stability was significantly greater with DACP than with its predecessor and was comparable to tear film stability without lenses. ${ }^{154}$ However, comfort data were not reported in this study, which brings into question whether there was any subjective improvement in reported in-eye comfort.

Hyaluronic Acid. Hyaluronic acid (HA), a hydrophilic glycosaminoglycan found throughout the human body, has been used in contact lens rewetting drops and in a range of artificial tear products to treat mild, moderate, and severe dry eye, ${ }^{155,156}$ and has been used as a novel internal wetting agent for contact lens materials. ${ }^{157-162}$ However, to our knowledge, HA has not been shown in any clinical studies to directly improve comfort associated with contact lens wear.

\section{Comparison Between Rigid and Soft Lens Materials}

At first glance, there is a considerable difference between the comfort associated with rigid and soft lenses. However, while this is true in the short term, there is little evidence that medium and long-term comfort is substantially different between them. Fonn and colleagues ${ }^{163}$ found no significant differences in ratings of comfort after 6 months between eyes of 27 patients fitted contralaterally with a soft and a rigid lens. However, average comfort was significantly lower for the eye wearing the rigid lens over the initial 3-month period. For the 16 patients who remained in the study for an additional period of 3 months, comfort between both eyes was reduced but remained only marginally lower for the rigid lens-wearing eye.

Morgan and colleagues ${ }^{164}$ were unable to find a difference in comfort between a group of adapted rigid lens wearers and soft lens wearers using lenses on a continuous wear basis. Maldonado-Codina and colleagues ${ }^{165}$ compared the comfort scores reported by subjects successfully wearing rigid or soft lenses on a daily wear basis with comfort reported by neophytes fitted with high oxygen transmission rigid lenses and silicone hydrogels on a daily wear basis for 2 weeks, followed by 11.5 months of continuous wear. Their results showed that, while neophytes in the SiHy group presented with a high comfort score from the very beginning, the rigid lens group reported significantly improved comfort scores over the first 2 weeks, remaining at the same level as the silicone hydrogels over the 12 months in continuous wear. ${ }^{165}$ Subjects who were experienced rigid lens wearers actually reported the highest comfort levels of all wearers, suggesting that long-term rigid lens wearers may ultimately be the "most comfortable" of all lens wearers.

Finally, Nichols and Sinnott ${ }^{8}$ and Ramamoorthy and colleagues $^{32}$ used a variety of statistical modeling approaches in a cross-sectional sample of 360 contact lens-wearing subjects to evaluate rigid lenses, compared with soft lens wearers, in predicting contact lens dry eye. Similar to other studies, rigid lens wear was not associated with a difference in predicting classification of subjects with or without contact lens dry eye.

In summary, there is little published evidence of a significant difference in the reported comfort between soft and rigid lenses in the long term, once the initial adaptation phase is complete. However, clinicians are aware of the fact that many RGP-wearing patients report increased comfort when they are switched into a soft lens, so this lack of evidence may relate more to the fact that such a study has not been conducted.

\section{Lens Design and Fit}

Soft Lens Design and Fit. The fact that soft contact lens fit can affect contact lens wearing comfort is supported by the practical experience of every contact lens practitioner. Moreover, it seems logical that a soft lens showing excessive movement or failing to cover the cornea will cause irritation through interaction between the cornea and edge of the lens. Nevertheless, few clinical studies have shed light on correlations between the subtleties of soft lens fit and comfort responses. The reasons for this are probably 2-fold: first, 
although many clinical trials have identified differences in comfort between lens types, only a few have systematically varied design parameters. Many clinical studies compare lenses of differing material as well as design. However, those studies that have sought to systematically evaluate lens design have tended to involve old, relatively thick designs in low EWC materials. ${ }^{166-169}$ Second, since so many factors affect contact lens-wearing comfort, it is difficult to control for all potentially confounding factors (e.g., modulus, thickness, and edge profile).

Corneal Coverage and Lens Diameter. An essential requirement of successful rigid contact lens fit is for the edge to stay clear of the cornea in all positions. Due to their inherent flexibility, the reverse is true with soft lenses. Since the lens edge rests against the eye and, due to stretching, exerts pressure, it has to overlap the relatively sensitive cornea to avoid discomfort. In order to allow for blink-induced movement, an overlap of at least $0.5 \mathrm{~mm}$ is assumed to be necessary. However, there appears to have been no work to confirm the optimum amount of overlap. A potential source of confusion is the fact that the true cornea is $>1 \mathrm{~mm}$ larger than the visible iris diameter ("white-to-white diameter"). ${ }^{170,171}$ Furthermore, the limbal transition zone varies between individuals and, thus, visible iris diameter is a poor predictor of corneal diameter. ${ }^{172}$

The pioneers of soft lens design assumed that the smallest acceptable lens diameter was optimal. But this was soon reassessed and early 12.5- and $13.5-\mathrm{mm}$ designs were replaced by larger lenses. ${ }^{173,174}$ One early study found that $13.5-\mathrm{mm}$ diameter lenses showed full corneal coverage in approximately $50 \%$ of eyes, while $12.5-\mathrm{mm}$ lenses gave total coverage in less than one-third of eyes. ${ }^{175}$ One study of tear replacement found an interrelation between lens diameter, comfort, lens movement, and "tear replenishment," with smaller diameter lenses giving reduced comfort, greater movement, and faster tear replenishment. ${ }^{176}$ However, these apparent interrelations are confounded by the fact that the lens diameters were relatively small (12.0-13.5 mm) and that their closeness to the typical corneal diameter is likely to have impacted comfort.

Most current soft contact lenses fall within the diameter range 13.8 to $14.2 \mathrm{~mm}$. However, since all lenses shrink when raised from room temperature to eye temperature, ${ }^{87,177}$ the diameter on-eye is quite different, being 13.54 to $14.16 \mathrm{~mm}$ in one study. ${ }^{87}$ It is possible, therefore, that some lenses are uncomfortable due to them being smaller than expected when placed on the eye.

Lens Movement and Base Curve. It is assumed that some lens movement is necessary in order to encourage postlens lubrication and, in turn, avoid mechanical irritation and corneal desiccation. However, there has been little or no research to examine the consequences of zero soft lens movement. Of perhaps greater interest to lens wearing comfort is the opposite situation of excessive movement. The conventional belief is that excessive movement causes discomfort through encroachment of the lens edge onto the cornea. However, an alternative explanation might be irritation through excessive interaction between the lens and the lids. It is difficult to quantify the true amount of lens movement as some movement takes place when the lids are closed. ${ }^{178}$

An extensive evaluation of soft lens fit reviewed more than 2000 contact lens fittings that had been classified as loose, tight, or optimal. ${ }^{36}$ Unacceptable fittings categorized as "loose" tended to show more movement and poorer comfort, with the mean comfort score for loose fittings being 7.4 compared with 8.5 for optimal fittings (on a 10-point scale). A relatively large proportion $(63 \%)$ of loose fittings were found to be less than comfortable $(<9$ on $0-10$ scale). Only one study has noted a significant correlation between lens movement and comfort, with less mobile lenses being rated more comfortable. ${ }^{179}$
Conventional contact lens practice assumes that the greatest influence on tightness of fit is back surface radius of curvature (base curve; BC). Most soft lens designs incorporate a single spherical curve on the back surface, although some soft lenses have utilized bicurve and aspheric designs. One of the most systematic evaluations of the effect of $\mathrm{BC}$ variation was undertaken with relatively thick, low EWC lenses; therefore, the findings have to be treated with some caution. ${ }^{180}$ Lowther and Tomlinson ${ }^{167}$ attempted to determine the minimum change in $\mathrm{BC}$ required to effect a significant change in various clinical outcomes such as vision, corneal edema, lens movement, and comfort. A change in $\mathrm{BC}$ of $0.95 \mathrm{~mm}$ was required to have a significant effect on comfort. A later study of midwater lenses found that a $0.6 \mathrm{~mm}$ flattening of $\mathrm{BC}$ resulted in significantly poorer comfort. ${ }^{181}$ A study with first generation SiHy lenses found improved comfort with $24 \%$ of patients by switching from an 8.60 - to an $8.40-\mathrm{mm}$ BC. ${ }^{182}$ However, the clinical picture is clouded by a proportion of the flatter lens fittings showing edge stand-off due to the relatively stiff material characteristics. Other studies of individual lens designs available in two BCs have found no significant difference in comfort when subjects were fitted with both BCs. ${ }^{183,184}$ However, this might be explained by the relatively small differences in $\mathrm{BC}$ in the products used in these studies (0.3 and $0.4 \mathrm{~mm})$.

Lens Centration and the Lens-Eye Relationship. It is assumed that soft lenses decenter in order to reach an equilibrium state that balances the various forces from the lids as well as the lens-ocular surface interaction. It seems unlikely that small amounts of decentration (e.g., $<0.3 \mathrm{~mm}$ ) are likely to affect comfort as this does not significantly alter the interaction of the lens with the cornea or the lids. However, in some cases there may be confounding factors, with centration being influenced by a factor that also affects comfort (e.g., looseness of fit). To our knowledge, no studies have examined the effect of minor changes in lens centration on comfort during lens wear.

Edge Alignment and Lens Edge Profile. The design parameters related to lens edge profile are less easy to specify as they encompass the thickness at various points near the edge and the actual shape of the edge profile. A study of lathecut low EWC lenses found no significant difference in comfort when the edge thickness was systematically varied between 0.08 and $0.16 \mathrm{~mm} .{ }^{168}$ Similarly, no significant difference was found in a study of high-water lenses varying in edge thickness from 0.12 to $0.24 \mathrm{~mm} .{ }^{169}$ However, these edge thicknesses are relatively thick compared with molded designs and it is possible that the range was not wide enough to detect differences.

Modern molded designs generally taper to a thinner edge than lathe-cut and older molded designs. Several edge shapes have been identified in the literature, including so-called "rounded," "knife," and "chisel" edges. ${ }^{64,185}$ In a study by Maissa and colleagues, ${ }^{64}$ the lens with the thickest edge shape (rounded) gave poorer comfort than one of the chisel edge designs and two of the knife edge designs. This rounded edge profile was also slightly less comfortable in the work by Hubner and colleagues. ${ }^{185}$ It is plausible that the thinner designs sit closer to the bulbar conjunctiva and have less interaction with the lids than the rounded design. Alternatively, since the lens types were of varying materials, it is possible that the relatively high modulus of the rounded design may also have been a factor influencing comfort. Evidence for the reduced lid interaction theory is provided by ocular coherence tomography (OCT) imaging. ${ }^{186-188}$ These show that thin, tapered edge designs show a smoother transition between the conjunctiva and the lens surface and produce less disruption ("buildup") of conjunctival tissue at the lens 
edge. ${ }^{187}$ Sharper, pointed edge designs also show less movement than thicker, rounded edges and induce more pronounced conjunctival staining. ${ }^{185}$

Another finding that may relate to edge fit comes from one study that found better comfort with a bicurve back surface design compared with a monocurve design, even though all other parameters were held constant, including sagittal depth and edge thickness. ${ }^{169}$ It is possible that the bicurve design afforded better alignment with the eye at the periphery of the lens, reducing localised pressure at this point.

Toric and Multifocal Designs. More sophisticated lens designs such as those incorporating prescriptions to correct astigmatism and presbyopia are thicker than spherical designs and this may affect comfort during wear.

Toric Contact Lenses. A study of contact lens dropouts found a disproportionately high number of astigmatic lapsed wearers. ${ }^{77}$ When, as part of the same study, these lapsed wearers were refitted with contact lenses, there was a higher failure rate with toric soft lenses than spherical lenses. In a recent survey of soft lens wearers, symptoms of dryness were more frequent among toric soft lens wearers ( $43 \%$ vs. $30 \%, P=$ $0.04){ }^{63}$ This mirrored the findings of a 1989 study that also found more symptoms of dryness in toric than spherical soft lens wearers $(40 \%$ vs. $13 \%, P<0.01) .{ }^{189}$ There is evidence that CLD from a variety of sources is often misinterpreted as dryness. ${ }^{65}$ The interaction of the lid margin with front surface irregularities may be difficult to distinguish from the interaction with a dry lens surface.

An early study of toric soft contact lenses systematically evaluated the clinical performance of toric designs of varying prism and truncation. ${ }^{190}$ Although comfort was assessed and contributed to the outcome variable of "overall acceptance," the comfort results were not reported separately. Not surprisingly, there was a tendency for the designs with thicker prism and more truncation to be less acceptable. Using a more recent prism ballasted, but non-truncated, design, Cho and colleagues found no significant difference in comfort between this and its spherical equivalent. ${ }^{191}$

Multifocal Soft Lenses. Clinical trials that evaluate multifocals in comparison with spherical soft lenses give some insight into possible effects on comfort from multifocal optical designs. However, there are few such studies. One study from 1990 found no significant difference in comfort, even though the lens was a diffractive multifocal incorporating optic zone on the back surface. ${ }^{192}$ A more recent study of a low EWC aspheric multifocal found no significant difference in symptoms compared with a single vision lens used for monovision. ${ }^{193}$

\section{Rigid Lens Design and Fit}

Rigid lenses are undoubtedly less comfortable initially than soft lenses. ${ }^{163,165,194}$ The discomfort arises from the interaction between the rigid edge of the lens and the eyelids, particularly the upper lid margin, as evidenced by the various strategies adopted to minimize the discomfort. ${ }^{195}$ Some rigid lens wearers reduce their blink rate or adjust their head posture to minimize the lid interaction. When fitting rigid lenses, practitioners often raise the patient's eyelids to alleviate the initial discomfort. The possibility that the cornea is an additional center of discomfort is suggested by piggyback soft-rigid lens combinations, which tend to be more comfortable than rigid lenses alone. ${ }^{196,197}$ However, this may be due to a cushioning effect that reduces the edge clearance of the rigid lens. The fact that the discomfort reduces when the eyes are held closed would also tend to refute this.

Three important factors relating to the edge of rigid lenses govern comfort; these are the thickness and shape of the edge and the amount of clearance from the cornea. The greater the edge clearance, the greater the interaction with the eyelids and, in turn, poorer comfort. ${ }^{198}$ Cornish and Sulaiman ${ }^{199}$ evaluated the effect of rigid lenses of varying center thickness ([CT], $0.10-0.21 \mathrm{~mm}$ ) on comfort and found that the thinnest design was actually the least comfortable, which was attributed to greater on-eye lens flexure. Mandell ${ }^{200}$ attempted to characterize edge shape by specifying the location of the edge apex and lens thickness at various distances from the edge, and found less comfortable edges tended to incorporate an apex close to the lens front surface. Shanks evaluated 13 edge shapes and noted differences in comfort between lenses, but came to no overall conclusion on the optimum shape. ${ }^{201}$ Korb and Exford ${ }^{202}$ proposed an alternative strategy for maximizing comfort. Rather than finishing the peripheral contour of the lens so as to allow the upper lid to slide easily over the lens, they modified the edge and periphery to encourage "lid attachment." The most systematic clinical evaluation of rigid lens edges was by La Hood, ${ }^{203}$ who assessed the comfort of four representative edge designs in four subjects: round, square, rounded anterior with square posterior, and square anterior with round posterior. The two designs with rounded anterior edges were significantly more comfortable than the other two. There was no significant difference in comfort between the lenses with square and rounded posterior edge profiles. The results confirm that the interaction of the lens edge with the eyelid is the most important factor in determining comfort in rigid lens wear.

Large Diameter Lenses. Large diameter RGP lenses might improve comfort by reducing lens movement and reducing the interaction of the lid with the edge of the lens. One potential classification for rigid lenses according to their overall diameter is "corneal" $(<12.5 \mathrm{~mm})$, "corneoscleral" (12.5-15.0 mm), and "scleral" lenses $(>15.0 \mathrm{~mm}) .{ }^{204}$

While there is clinical evidence of the short-term improved comfort with corneoscleral and scleral lenses compared with corneal RGP lenses, few well-controlled studies have addressed this point. Sorbara and Mueller ${ }^{205}$ compared the comfort of RGP lenses with different overall diameters in a nondispensing study in patients with keratoconus. The authors concluded that smaller diameter lenses $(8.7$ and $9.0 \mathrm{~mm})$ were initially more comfortable in central cones, while larger lenses (10.1 and $10.4 \mathrm{~mm}$ ) were preferred in oval cones. According to their results, lens movement was not directly related to comfort.

To date, there is no solid evidence of the benefits of corneoscleral and scleral lenses compared with corneal RGP lenses in terms of comfort, beyond clinical intuition. The average comfort ratings reported by some authors for scleral lenses (with diameters from 18.0-25.0 mm) fitted to patients with several ocular surface diseases ${ }^{206}$ are in the same range as comfort values already reported for corneal lenses in healthy subjects. ${ }^{163,165}$ Nevertheless, a direct comparison cannot be drawn, as most of the corneoscleral lenses were prescribed for eyes with serious eye disease.

In conclusion, considering the growing interest in the use of corneoscleral and scleral rigid lenses for eyes that do not exhibit disease or abnormal surface profiles, it is clearly necessary to conduct well-controlled, randomized studies where the potential for enhanced comfort of these lenses over standard diameter rigid lenses is investigated.

Tear Exchange. Placement of a contact lens on the eye leads to disruption of the tear film and to stagnation of the postlens tear layer during soft contact lens wear. ${ }^{207,208}$ Liberal exchange of this layer is generally considered preferable because it more closely represents the natural free flow of tears when no contact lens is in place and because buildup of debris behind the lens has been anecdotally associated with increased likelihood of corneal inflammatory events. ${ }^{209-214}$ 
Measurement of tear exchange is almost exclusively conducted by determining the expulsion of a "marker" of some sort from behind the lens, which is typically sodium fluorescein, using a technique called fluorophotometry.

McNamara and colleagues ${ }^{176}$ used fluorophotometry to measure tear exchange in 23 subjects while they wore lenses of four different diameters in separate 30-minute wearing trials. Increased tear exchange was accompanied by decreased comfort, although intuitively the effects are linked and both are the result of decreasing lens diameter. Paugh and colleagues $^{215}$ found fluorescence decay over 30 minutes to be greater with a prototype lotrafilcon A contact lens than thinner, less mobile etafilcon lenses in 11 subjects, but found no significant correlation between lens comfort and tear exchange rate.

Lin and colleagues ${ }^{216}$ investigated the effect of scalloped microchannels on the posterior surface of contact lenses on tear exchange over a 30-minute period, measuring comfort concurrently. They theorized that the channels might lead to increased tear exchange and were able to show this in Asian, but not in non-Asian, subjects. The microchannels do not induce any discernible change in comfort during the relatively short wearing times for which this design has been studied. ${ }^{216,217}$ One further method that may increase tear exchange is to fenestrate lenses, which would increase the flow of tears from the back surface of the lens to the front surface. To date, no studies appear to have investigated tear exchange with fenestrated soft lenses, but one $\operatorname{paper}^{218}$ was able to demonstrate that such a procedure does result in markedly reduced comfort due to interactions between the palpebral conjunctiva and the fenestrations.

The above methods of achieving increased tear exchange all involved increased lens movement, which is the likely associated factor. Unfortunately, increased lens movement is also associated with decreased comfort. The conventional view and some evidence is that tightly fitting lenses are comfortable and that loose-fitting lenses are likely to be less comfortable than well-fitting lenses. ${ }^{36,77}$

In summary, there is little evidence that increasing tear exchange will have a positive effect on lens comfort and, to the contrary, changes to lens parameters that may bring about increased tear exchange are likely to have a simultaneous negative impact on lens comfort.

\section{Miscellaneous Factors}

Tinted Lenses. The tints on soft contact lenses can be translucent or opaque and in general, three types of tint are commonly used: visibility (or "handling") tints, enhancement tints, and those with an opaque (or semiopaque) tint. Opaque tints can be applied using dot matrix printing on the lens surface, ${ }^{219}$ which can result in a relatively rough surface, ${ }^{220,221}$ and one study comparing these lenses with their clear equivalent found increased discomfort with the colored lenses. ${ }^{221}$

Manufacturers have tried to overcome this problem by either housing the tint within the lens itself in the form of a laminate or applying a hydrophilic "coating" layer. ${ }^{220}$ The laminate construction has the advantage of encapsulating the printed matrix, but leads to increased lens thickness, ${ }^{222}$ which in turn may have a detrimental effect on comfort. Opaque lenses also have a fixed pupillary aperture that may lead to the wearer being more aware of these lenses due to constrictions of the visual field, ${ }^{223,224}$ peripheral vision blur, ${ }^{225}$ and so-called "annular tinted contact lens syndrome" where subtle distortions of the cornea and induced astigmatism have been observed, with a subsequent reduction in vision. ${ }^{222,226}$ These visual problems may have an impact on the perceived comfort of the contact lenses, since problems with vision appear to affect comfort (Papas E, et al. IOVS 2003;44:ARVO E-Abstract 3694). In contrast, Gauthier and colleagues ${ }^{225}$ compared opaque colored lenses with their clear equivalents and observed no difference in overall comfort. Thus, evidence remains contradictory in terms of impact of lens tinting on comfort in contact lens wear.

Indicator Markings. Since most soft lenses are castmolded, indicator markings on these lenses are placed onto the metal mold inserts by techniques such as electric discharge, diamond point engraving, and laser etching. The importance of the form of these markings to comfort have been the subject of various patents, with some placing much importance on these markings being composed of individual dots no greater than 90 $\mu \mathrm{m}$ in diameter being recessed into the lens front surface by a depth of 2 to $10 \mu \mathrm{m} .{ }^{227,228}$ There is no published literature to date relating lens markings to in eye comfort, but anecdotal reports have occurred of lens wearers reporting increased lens awareness when lens markings have been added to or changed for existing products. In addition, anecdotal reports exist of these markings becoming filled with tear film components, ${ }^{229}$ and these deposits could act as a source of irritation.

\section{Contact Lens Deposition}

Since the commercialization of soft lenses in the early 1970s, clinicians have realized that contact lens materials rapidly attract tear film contaminants and that this deposition impacts lens performance. ${ }^{230,231}$ While intuitively it would appear obvious that there would be a link between comfort and contact lens deposits, proving such a link is somewhat more challenging, as many studies rely on visible deposition rather than biochemically measuring the actual degree of deposition. Visible measures of deposition are usually done either on-eye at the slit lamp biomicroscope or off-eye using various versions of the RUDKO scale, first reported by Allergan in the mid1970 s. $^{232}$ This is problematic, as it is known that visible and measured deposition show a poor correlation. ${ }^{233,234}$

Visible Deposits and Comfort. Roughly half of the studies conducted to date investigating comfort and its link with deposition have used visible deposition rather than biochemical analysis of deposits (Table 2).

The earliest of these studies by Nilsson and colleagues ${ }^{235,236}$ showed that lenses with the greatest level of deposition were generally less comfortable $\mathrm{e}^{235}$ and that the use of a weekly enzyme cleaner resulted in increased comfort. ${ }^{236}$ While no direct correlation between comfort and deposition was reported in either paper, each surmised that increasing deposition was an important factor in reducing lens comfort. However, one study conducted at around the same time ${ }^{237}$ and two later studies ${ }^{238,239}$ were unable to demonstrate any correlation between visible deposition and comfort.

A larger multicenter study ${ }^{240}$ investigated lenses that were replaced every day versus those worn for up to 1 year. The daily disposable lenses, not surprisingly, exhibited reduced deposits over the course of the study and exhibited higher levels of comfort. The investigators linked these two factors, but many other factors (e.g., surface wettability; care solution effects) could have been the major reason for the improved comfort. Two other studies ${ }^{241,242}$ conducted at a similar point in time looking at the impact of frequent replacement of lenses on subjective performance were also able to link reduced levels of visible deposits with improved comfort. Of these, only one study ${ }^{241}$ actually reported a correlation between subjective responses and deposition, and while the correlation was weak $(r=-0.33)$, it was statistically significant. The remaining five studies (Truong TN, et al. IOVS 2008;49:ARVO E-Abstract $4833)^{44,54,243,244}$ all included SiHy materials that were replaced 
in 4 weeks or less. Of these, four reported no correlation between visible deposits and comfort 44,54,243,244 and the only piece of work to suggest such a link exists is a conference abstract (Truong TN, et al. IOVS 2008;49:ARVO E-Abstract 4833) using a retrospective analysis of short-term wear of various lenses.

In summary, studies conducted using visible methods to determine lens deposition have provided poor evidence that comfort and deposits are linked, particularly over the 1 month or less that lenses are now typically worn. Future studies would be ill advised to rely on visible assessment of deposition to relate to contact lens comfort.

Quantified Protein Deposits and Comfort. To date, a dozen studies have used various biochemical analyses to quantify protein deposition and attempt to link it with contact lens comfort, and these are summarized in Table 3.

Of the nine studies looking at hydrogel materials alone (investigating all FDA categories), eight were not able to show any correlation between the quantity of protein deposited and comfort. ${ }^{11,245-251}$ The only exception to this was a study by Lebow and Christensen ${ }^{252}$ that investigated the impact of two care systems (one using a daily cleaner) on protein deposits and comfort in 76 subjects wearing ionic, high EWC lenses. They found that the subjects using the daily cleaner reported improved comfort and that the lenses collected from these subjects showed reduced protein (lysozyme) deposition. No actual correlation analysis was reported, but the authors surmised that the reduced deposition and comfort were

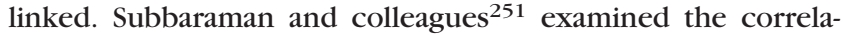
tion between symptoms and protein deposition over an 8-hour period in 30 subjects using an FDA Group IV material; while they were unable to report any significant correlation between total protein and symptoms, they were able to show a correlation $(r \geq 0.64 ; P<0.001)$ between reported comfort and the amount of denatured lysozyme. They concluded that while comfort cannot be linked with quantity of protein deposited, it might be related to the amount of denatured protein on the contact lens.

Of the three studies investigating protein deposition and comfort when subjects wore SiHy materials, the results were equivocal. One study ${ }^{253}$ showed no relevant correlation, while another ${ }^{254}$ showed that a rewetting drop resulted in increased comfort and reduced total protein, total lysozyme, and increased protein activity and thus a relationship was surmised. The most recent study on a large sample of SiHy lenses was able to show a weak correlation $(r=-0.13)$ with comfort on insertion. ${ }^{255}$ To date, no study has investigated the link between denatured protein and comfort for SiHy materials, but one in vitro study ${ }^{256}$ has reported that lysozyme activity does reduce over time following its deposition on both hydrogels and silicone hydrogels.

In summary, the main conclusion is that the amount of deposited protein appears unrelated to contact lens comfort, but protein activity may be correlated. However, further work in this area-particularly with regard to the degree of activity of proteins other than lysozyme-is required to confirm any such relation.

Quantified Lipid and Mucin Deposits and Comfort. The final area linking contact lens deposition and comfort are those quantifying the amount of lipid or mucin deposition, and these five studies are summarized in Table 4.

The two studies reporting on mucin deposition (one looking at hydrogel materials only ${ }^{257}$ and the other including silicone hydrogels ${ }^{258}$ ) were both unable to link the amount of mucin deposition with comfort. This lack of association was also reported for lipids in two studies looking at hydrogel materials. ${ }^{11,247}$ A more recent study investigating lipid deposition on a variety of SiHy materials was able to show weak (but significant) correlations for both overall comfort $(r=-0.13 ; P=$ $0.03)$ and comfort on insertion $(r=-0.16 ; P=0.008) .{ }^{255}$

In summary, the evidence-linking lipid or mucin deposition with contact lens comfort is either nonexistent or weak and future work should, perhaps, be directed at investigating mucin or lipid breakdown products rather than total lipid or mucin, if such a link is to be established.

\section{Wearing Modality}

Lens Wearing Schedule (Daily, Flexible, Extended, or Continuous Wear). Comparison of comfort differences between daily wear; flexible wear (occasional overnight wear); extended wear (regular overnight wear for up to 6 sequential nights); and continuous wear (regular overnight wear for over 6 sequential nights) of contact lenses is difficult because of numerous confounding factors. By virtue of the known average difference in comfort between contact lens wearers and nonwearers, a greater degree of discomfort on awakening is to be expected in those who sleep in lenses. Differences between daily wear comfort and comfort after sleep may be a function of hypoxia or tear disturbances rather than the wear schedule. Further, those who sleep in their lenses may selfselect or survive in that modality on the basis of comfort, and prolonged wear such as extended wear means a longer wearing period and comfort differences may be related to the exposure time rather than the modality.

A number of studies have compared comfort between daily wear and extended wear of hydrogel lenses. Poggio and Abelson $^{259}$ conducted a historical cohort study of 2433 cosmetic contact lens wearers. They reported that users of disposable extended wear lenses reported symptoms less frequently at routine visits than users of nonreplaced hydrogel daily wear lenses. It is important to note that the lens materials and designs and replacement frequencies were different in the two groups, meaning that the comfort difference can be not attributed to the wearing schedule in isolation.

Nichols and colleagues ${ }^{260}$ conducted a randomized, crossover, dispensing clinical trial specifically for the purpose of comparing daily disposable and disposable extended-wear modalities, using commercially available etafilcon brands. There was no significant difference between DD and EW in terms of lens comfort and awareness. However, a significant number of patients reported increased levels of ocular discomfort and irritation in the morning while in the extended-wear modality. Despite this, the subjects preferred the extended wear option overall, on the basis of convenience. The study by Aakre and colleagues ${ }^{261}$ followed 49 successful DD wearers, with 19 continuing to wear DD hydrogel lenses and 30 refitted with SiHy lenses on a 30-day/night schedule over 6 months. They were unable to demonstrate differences between the two modalities in terms of comfort and dryness.

Chalmers and colleagues ${ }^{48}$ looked at previous daily and EW of hydrogel lenses and its impact on symptoms with wear of lotrafilcon A SiHy lenses. The study feature of interest here, in terms of comparing wearing schedules, is that at baseline there was more than double the number of subjects who had previously worn hydrogel lenses in daily mode that experienced end-of-day dryness often or every day and who experienced moderate or severe end-of-day dryness compared with those who had worn hydrogel lenses in EW. This may be the result of a difference in the material and design of lenses prescribed for daily and EW, a direct protective effect of EW brought about by, for example, corneal hypoxia, or a bias phenomenon brought about by self-selection or survival. Santodomingo and colleagues compared DW and CW of each of two SiHy lenses over 18 months and found little evidence of major difference in symptoms of comfort and dryness between 


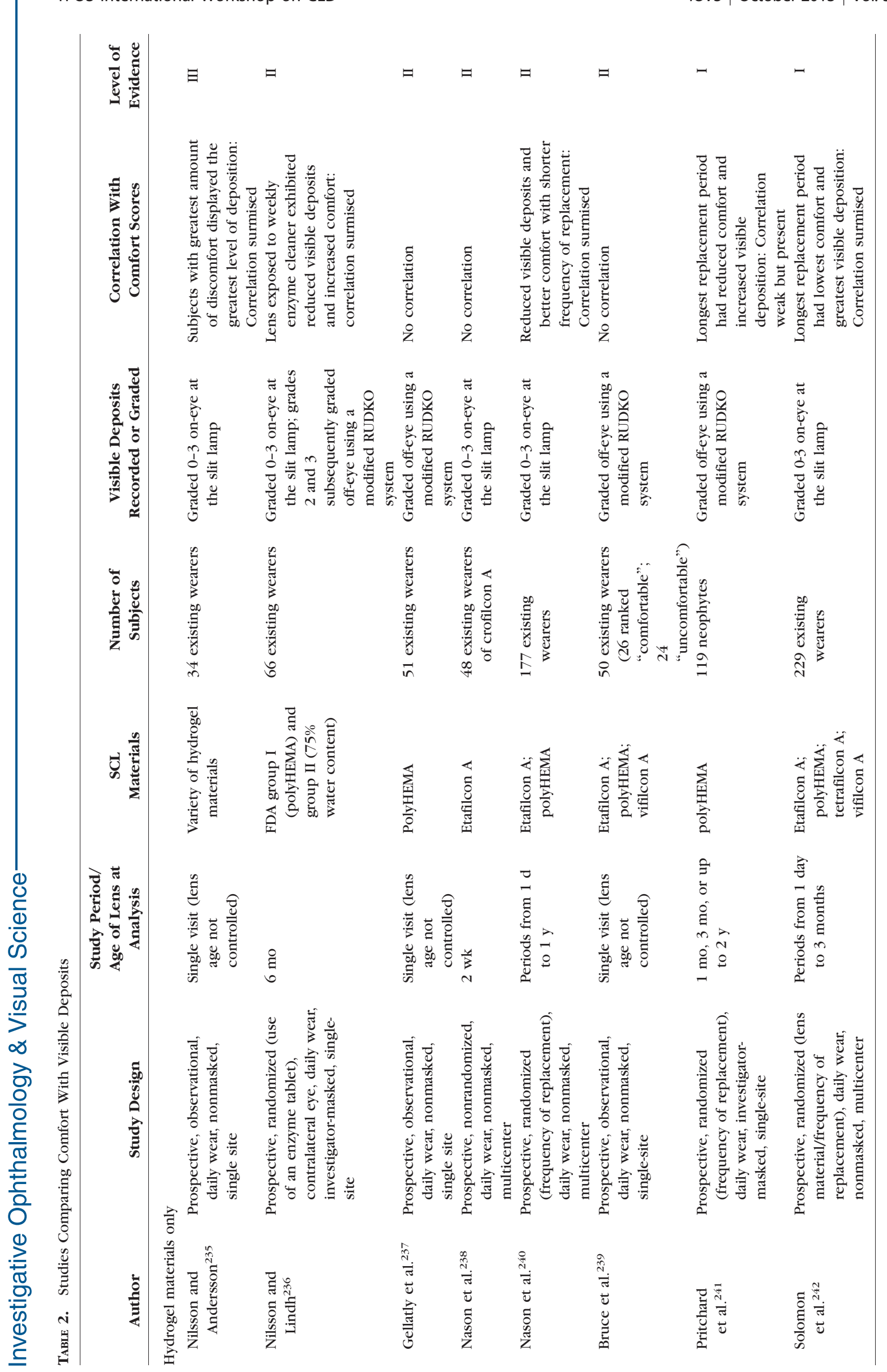




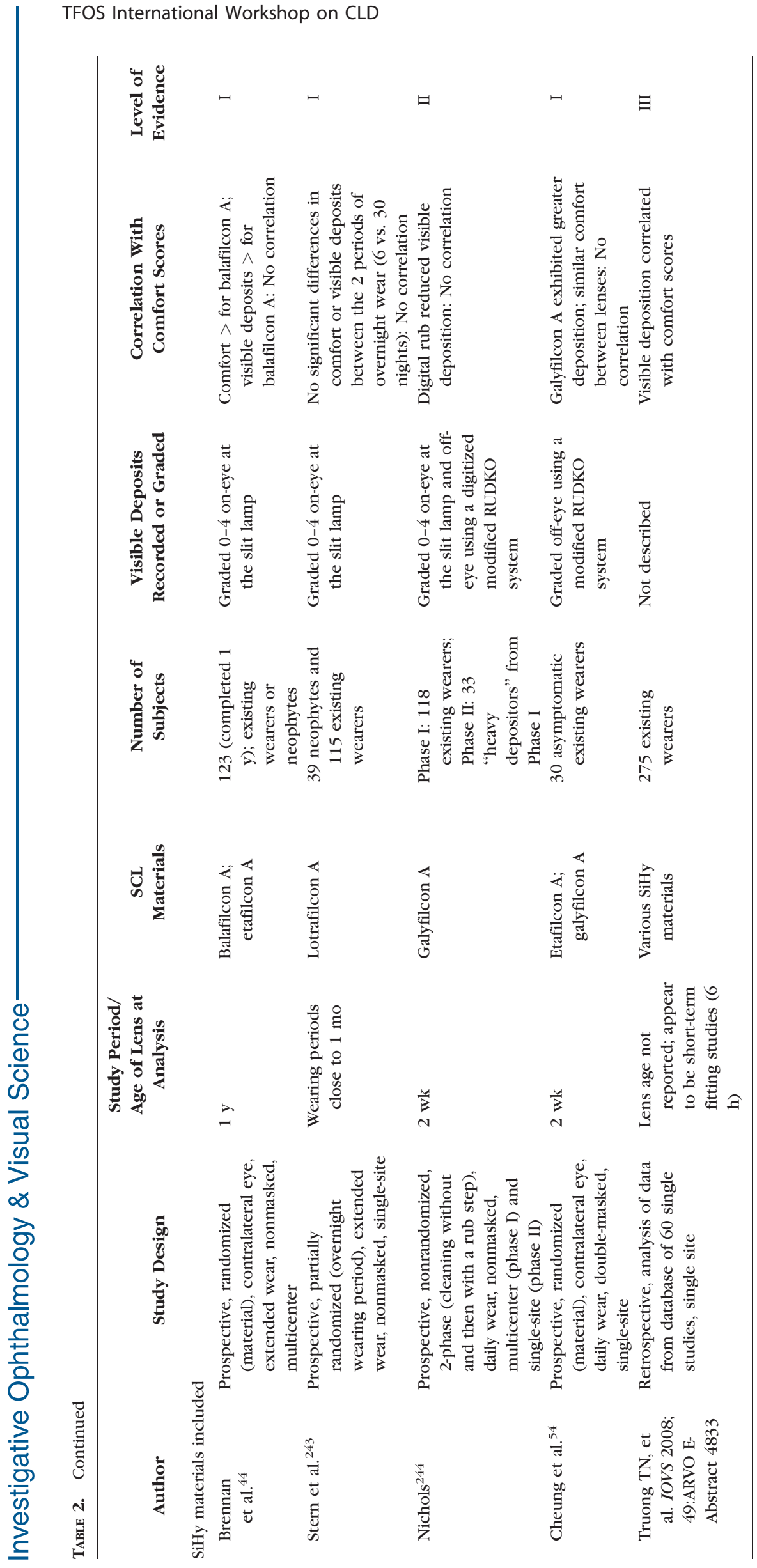




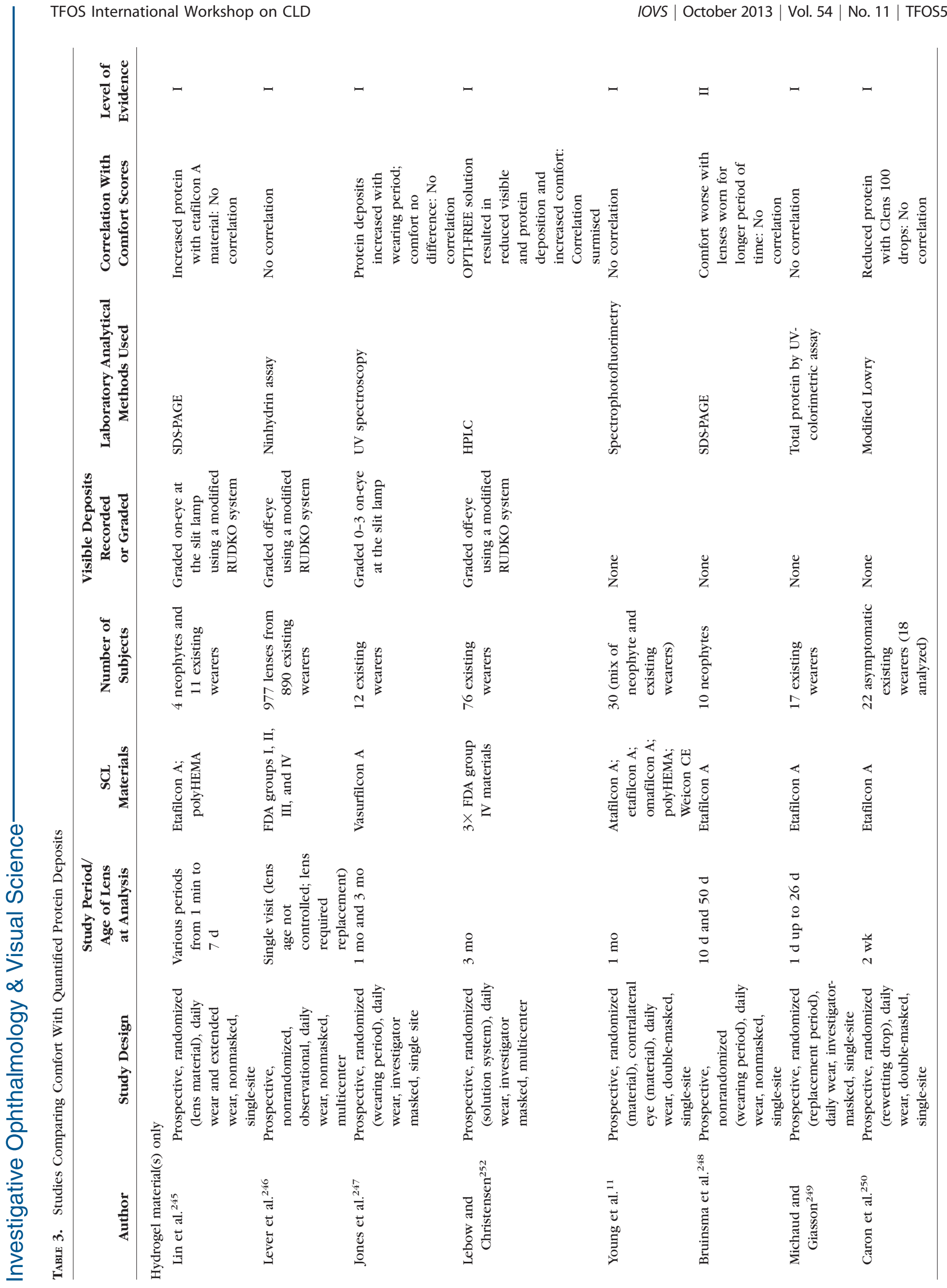




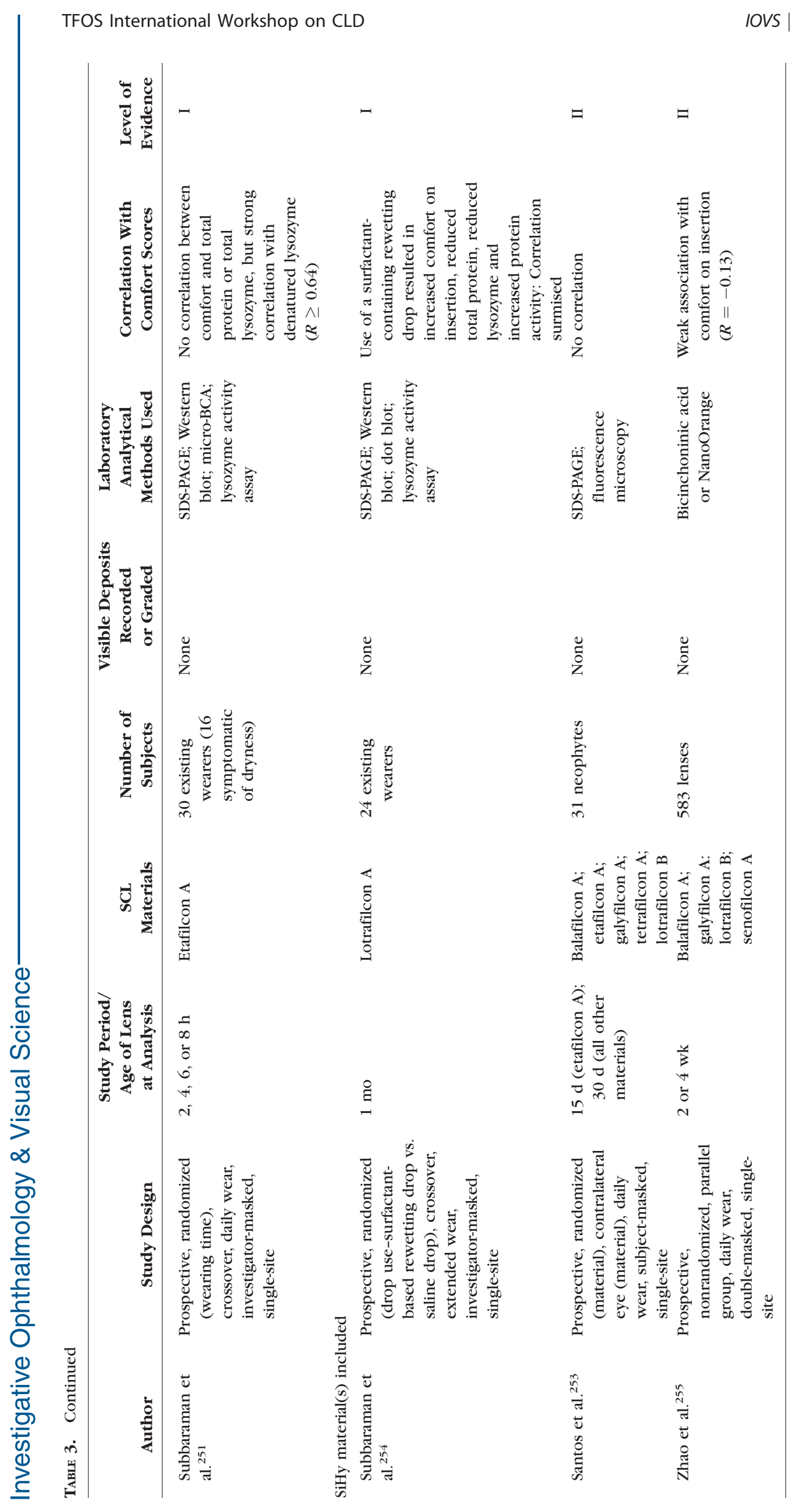




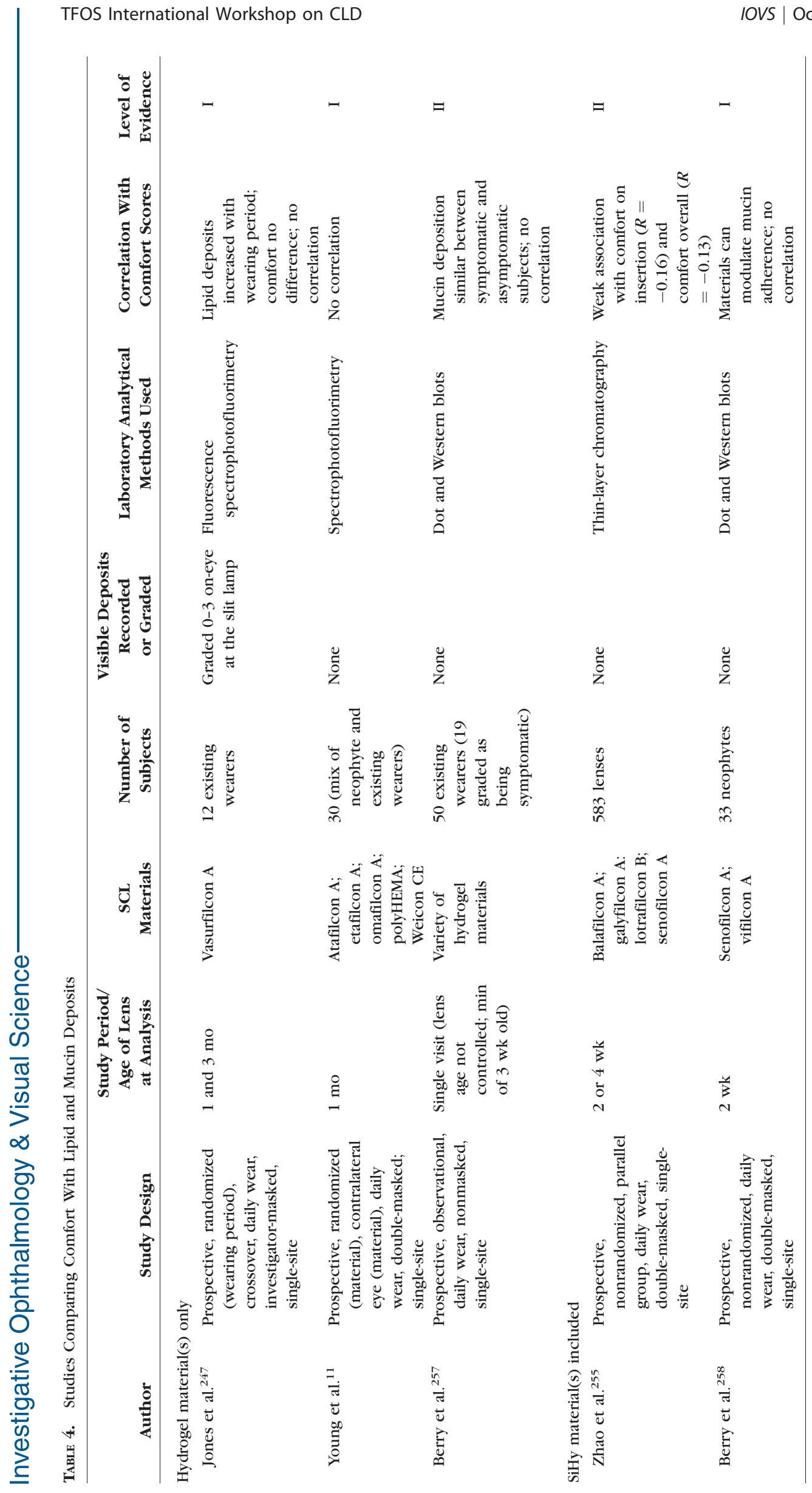


the two wearing schedules. ${ }^{50}$ Bergenske and colleagues ${ }^{55}$ reported on 317 subjects wearing SiHy lenses in CW and compared them with 81 neophytes introduced to hydrogel lens DW in a prospective, 3-year, open-label, nonrandomized study. They found that wearers of the hydrogel lenses reported during-the-day and end-of-day dryness more frequently. Ramamoorthy and colleagues ${ }^{106}$ considered a range of factors that might be associated with contact lens-related dry eye in a cross-sectional study. This study considered both SiHy and hydrogel lens wearers combined. Those who wore their lenses overnight were almost one-third as likely to be classified as having "dry eye" as those who did not, in a univariate model. However, a model that controlled potential confounding factors (age, sex, recent contact lens refitting, and number of weekly applications of artificial tears/rewetting drops) did not show overnight wear to be a significant factor.

As noted earlier, the wearing modality may interact with the effect of oxygen on comfort, if such an effect exists. Perhaps surprisingly, a tentative conclusion can be drawn that, apart from relatively minor dryness upon awakening, individuals who sleep in lenses are not at a disadvantage and indeed may benefit in terms of comfort and dryness compared with those who do not sleep in lenses and wear them on a daily wear basis. There have been no studies reporting superior comfort in DW, save for comfort on awakening. To date, inadequacies of study designs prevent more definitive conclusions from being drawn.

Duration of Wear. The comfort and dryness response to contact lens wear may not be static. Certainly, practitioners and patients are familiar with comfort adaptation to gaspermeable lenses. Fonn and colleagues ${ }^{163}$ and Morgan and colleagues ${ }^{164}$ have both tracked the dramatic change in comfort that occurs during the initial few weeks of wear. SiHy lenses are generally stiffer than hydrogel lenses and may also have surfaces with higher coefficient of friction, as discussed earlier. It is reasonable to propose that a similar, albeit lesser, adaptation may occur during wear of these lenses as takes place during the initial wear of gas-permeable lenses. Certainly, while the first week of wear may show the largest adaptation to comfort, the study of Chalmers and colleagues ${ }^{48}$ suggested that there is a gradual decrease in the percentage of subjects with more severe dryness frequency and also with moderate or severe dryness over 6 to 12 months while wearing SiHy lenses in $\mathrm{CW}$ mode.

The impact of duration of wear may also explain in part the differences observed between studies when comparing hydrogels and silicone hydrogels. In short-term studies (up to 30 days) particularly for DW, hydrogels would generally seem to provide equal or superior comfort. ${ }^{47,54,59,64,116}$ In longer-term studies, silicone hydrogels would appear to provide better comfort. ${ }^{44,45,48,55}$

A confounding and possible competing factor in considering the impact of duration of wear on comfort is the effect of lens replacement frequency. There does not appear to be any Level I evidence to support the contention that duration of wear is important in determining contact lens comfort

Lens Age and Replacement Frequency. Lens age is an obvious candidate for influencing CLD, as lenses begin to attract tear film components immediately upon application to the eye. ${ }^{245,262-264}$ Complexing and denaturation of this material can then lead to potentially problematic deposits on and within the lens. ${ }^{265,266}$

In an early cross-sectional study, Brennan and Efron ${ }^{189}$ found that increasing lens age led to increased frequency of dryness, but they were only able to separate differences between lenses younger and older than 6 months that had been worn on a conventional (nonplanned replacement) basis. The move toward disposable lenses in the late 1980s was an initiative to limit the amount of deposition, in the hope of diminishing complications. ${ }^{267,268}$ Frequency of replacement of contact lenses thus becomes highly relevant as a factor affecting contact lens wearing comfort and also offers a framework to examine the effect of lens age on comfort.

A series of studies discussed below identified improved comfort with disposable or frequent replacement reusable lenses and, furthermore, with increasing frequency of lens replacement. However, it is important to remember that the lenses used for this purpose are not necessarily made from the same lens material or with the same design as the comparator lenses or cared for with the same system. The imperfect control in such instances leaves open the possibility that it is not the lens replacement frequency as much as these other factors that are responsible for the observed improvements in comfort. A further challenge faced by researchers is achieving appropriate masking with respect to the replacement frequency in dispensing studies. Table 5 lists studies that allow the effect of lens age or different replacement frequencies on comfort to be compared, along with an indication of the quality of the studies in conforming with the evidence-based principles adopted within this report.

Boswall and colleagues conducted a retrospective chart review at a single contact lens practice from extended wear patients, of whom 65 wore disposable (7- to 14-day replacement) contact lenses and 61 wore nonplanned replacement lenses. $^{270}$ They found severe symptoms (itching, burning/ drying, and foreign body sensation) to be reduced in the disposable group, implying that increasing lens age is a factor in producing such symptoms. However, the average wearing time each day for the nonreplaced lenses was longer, presenting a possible confounding factor. Poggio and Abelson's ${ }^{259}$ historical cohort study compared disposable extended wear with nonreplaced daily and extended wear lenses. They found that disposable extended wear contact lens users reported symptoms less frequently at scheduled visits than both conventional daily wear and conventional extended wear users and that they had a lower rate of unscheduled visits for symptoms.

Poggio and Abelson's ${ }^{271}$ historical cohort study of 1954 daily wearers of soft contact lenses found that those using reusable, frequently replaced lenses had a significantly lower reported frequency for symptoms (particularly grittiness, scratchiness, irritation, and pain) compared with nonplanned replacement conventional wearers. In 1996, Pritchard and colleagues $^{241}$ randomly assigned 119 neophytes to either a 1or 3-month replacement schedule or nonreplacement group while wearing thin $38 \%$ EWC polyHEMA contact lenses. While there were reduced complications with the more frequently replaced lenses, ratings of comfort and overall satisfaction were not found to be different between the groups. Potential reasons for there being no difference include the possibilities that replacement frequency does not influence comfort, replacement frequency is less important in thin low EWC lenses, their technique lacked sensitivity to measure such a difference in this population, or subject bias induced by their knowledge of how often they replaced their lenses.

Relatively few studies have compared the comfort advantage of 2-week versus 1-month replacement and there are certainly no Level I evidence that make this comparison. Malet and Schnider ${ }^{275}$ reported a prospective study of 3066 daily wearers of monthly replacement lenses who were refitted at over 300 individual practices into a 2-week replacement regime. This observational study concluded that subjective comfort was improved by reducing replacement intervals to 2 weeks. The study made a particular point in identifying that the improved subjective comfort was also dependent on a compatible lens cleaning regiment. ${ }^{275}$ 


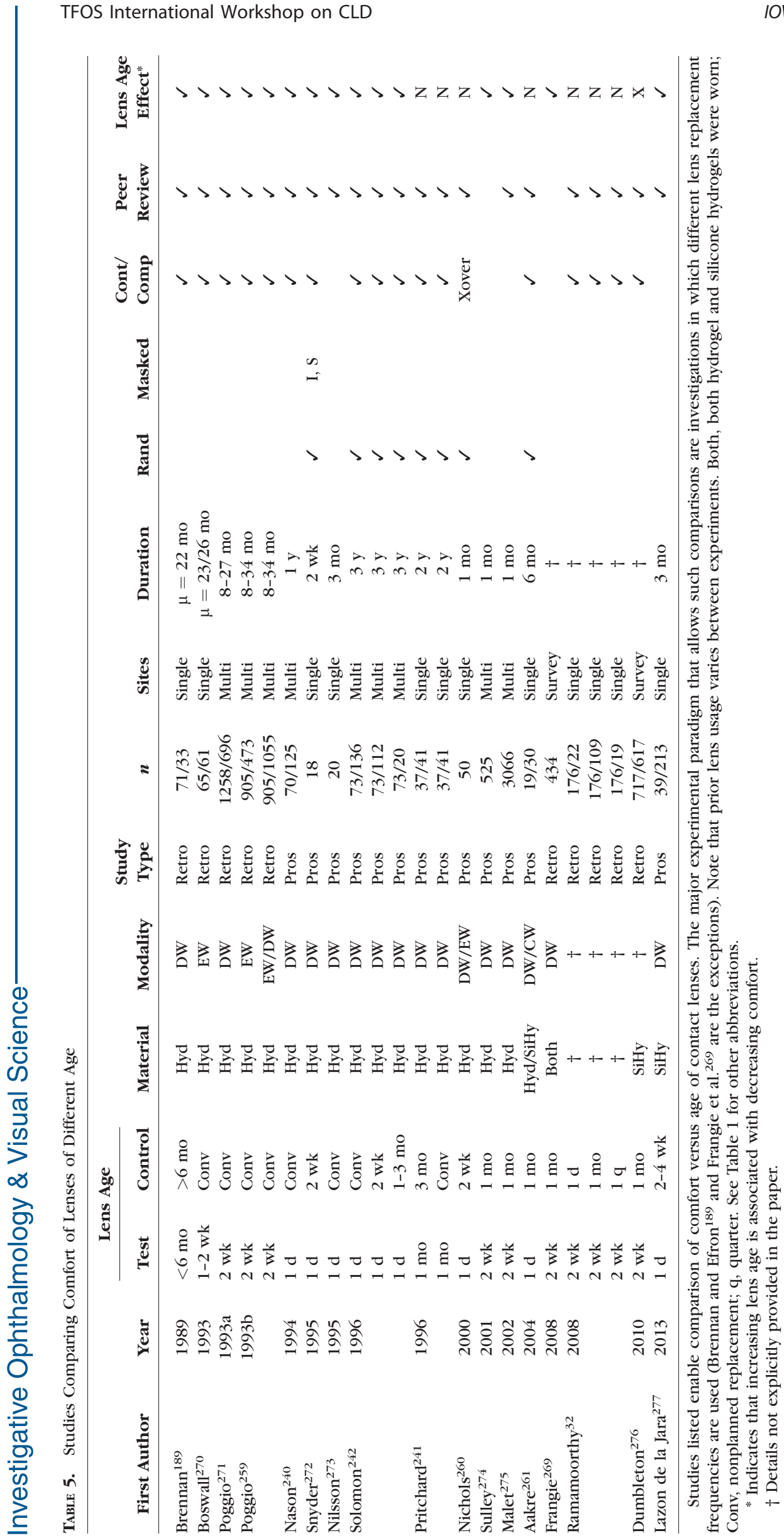


Sulley and Meyler's study ${ }^{274}$ - with a similar designyielded similar results. In both studies, the lens materials and designs were different between the replacement frequency groups, subjects were not masked, and there was not a concurrent control lens run alongside the 2 -week replacement lenses.

In a conference abstract, Jones and colleagues ${ }^{278}$ reported that subjects replacing their lenses on a daily disposable or 2week schedule were less likely than monthly wearers to report dryness. Frangie and colleagues ${ }^{269}$ surveyed 271 and 163 patients wearing a variety of monthly replacement hydrogel and SiHy lens brands, respectively. A total of $68 \%$ of the hydrogel wearers and $71 \%$ of the SiHy wearers noticed a decrease in comfort during the month. Less than $10 \%$ of both groups noticed that this occurred within the first 2 weeks of the wearing period, with the remaining $90 \%$ finding that the discomfort developed in the third and fourth weeks of the month. While they did not provide statistical analysis, Long and colleagues $^{61}$ showed data that supports the concept that lenses after a month of wear are less comfortable than after 2 weeks of wear.

One study stands in contradiction to the general trend of reports that have found shorter replacement schedules lead to better comfort. Dumbleton and colleagues ${ }^{276}$ conducted a survey of 1344 wearers of SiHy contact lenses through practitioners in the United States, with approximately half wearing lenses with a manufacturer's recommended 2-week replacement schedule and the other half on a monthly schedule. Noncompliance was found to lead to lower comfort, perhaps expectedly. After adjusting for compliance, the authors reported modest but statistically significantly better comfort with the monthly replacement lenses compared with the lenses recommended for a 2-week modality. The study surveyed existing wearers and so selection and survival bias cannot be ruled out in addition to differences in lens materials and design.

Most of the remaining studies considering the impact of replacement frequency have looked at the effect of daily disposables on lens comfort. Early hydrogel lens studies considered the 1-day replacement modality against conventional lens replacement. ${ }^{240,242,273}$ Daily replacement led to a number of benefits, including improvements on a range of comfort measures. Daily disposability also led to a range of comfort benefits against lenses planned to be replaced on a 1to 3-month basis. ${ }^{242}$ Solomon and colleagues ${ }^{242}$ also found benefits of a daily versus a 2 -week replacement schedule. It is interesting to note that the single study investigating the impact of replacement frequency on comfort, which conforms to the gold standard principles of a controlled, randomized, masked study did not find a difference between daily and two weekly replacements. ${ }^{272}$ However, it is not clear in this study whether the method for measuring comfort was adequate to detect differences, whether the sample size $(n=18)$ was sufficiently powered to detect a difference, whether lens care influenced comfort, and whether the lens type used was not resistant to the effects of aging.

The potential comfort advantage of daily disposal of SiHy lenses versus other replacement schedules has not been widely studied. In the one available study to-date, Lazon de la Jara and colleagues $^{277}$ reported that end-of-day comfort and dryness ratings were significantly better for daily disposable wear than when the same material and design was used in a reusable manner with either hydrogen peroxide or multi-purpose care systems.

Two studies have compared daily disposable lenses with extended or continuous wear. In the first, daily disposable hydrogel lenses were compared with a 2 -week replacement hydrogel. $^{260}$ There was no difference between the daily disposable and extended wear modalities with respect to subjective responses, except that those sleeping in their lenses found comfort on awakening to be inferior. In the second study, daily disposable hydrogel lenses were compared with monthly replacement silicone hydrogels in continuous wear. ${ }^{261}$ There were no differences of note between the two groups.

A final study looking at the effect of a variety of replacement intervals on lens comfort was conducted by Ramamoorthy and colleagues. ${ }^{32}$ Individuals $(n=360)$ were surveyed with the CLDEQ and categorized as either having or not having dry eye. Daily, 2-week, monthly, and quarterly replacement schedules were all represented in the sample, as were both hydrogel and SiHy lenses. Replacement schedule was not found to be predictive of how the subjects were classified. However, it is not clear that the study was powered to make determinations with respect to the different schedules and to what extent selection and survival bias were influential.

In summary, there is almost a complete absence of masked, randomized, controlled studies that consider the impact of replacement schedule on comfort and dryness, preventing a definitive statement being made on the topic. Nonetheless, there is a tendency for the studies that provide circumstantial evidence regarding replacement schedules to suggest that replacement that is more frequent is conducive to greater comfort.

Time of Day. End-of-day dryness and discomfort arguably represent the most challenging issue for the contact lens industry today. While discomfort is the major reason cited for contact lens discontinuation, ${ }^{77,78}$ a breakdown by the exact nature of discomfort is illuminating. Chalmers and Begley ${ }^{279}$ studied responses to a questionnaire of 1054 patients who presented for eye care in a multicenter cross-sectional study. The leading causes of discontinuation among the 167 former contact lens wearers in their sample were dryness and end-ofday discomfort, which were cited by $41.9 \%$ and $38.3 \%$, respectively.

Begley and colleagues ${ }^{280}$ surveyed 367 unselected contact lens wearers in their 2001 cross-sectional study of North American optometric clinical practices, finding that the percentage reporting moderate to intense ocular discomfort increased from $19 \%$ in the morning to $56 \%$ in the evening. The frequency of dryness among the 367 contact lens wearers in the group was significantly higher late in the wearing day compared with earlier in the day, with an increase from $12.7 \%$ who reported moderate to intense dryness in the first 2 hours of wear to $28.5 \%$ late in the day. ${ }^{279}$ In an analysis of questionnaire responses from 84 clinical sites in North America, Chalmers and colleagues ${ }^{60}$ found that between $3 \%$ and $15 \%$ of subjects, depending on age and type of lens material worn, reported end-of-day discomfort ("extreme" or "very") and between $7 \%$ and $24 \%$ report end-of-day dryness. Young and colleagues ${ }^{63}$ reported severity of end-of-day dryness to be of sufficient significance to categorize subjects as having "contact lens-associated dry eye" in $31 \%$ of wearers.

While noticed even in nonwearers and spectacles wearers, all symptoms-but particularly end-of-day dryness-are more pronounced in contact lens wearers. ${ }^{279-281}$ Further, contact lens wearers report markedly fewer symptoms without the lenses in place: for example, only $1.5 \%$ report moderate to intense late-day dryness according to Chalmers and Begley. ${ }^{279}$

Evidence in support of decreased comfort toward the end of the day necessarily cannot arise from Level I studies, as it is virtually impossible to conduct a controlled, masked, randomized study where time of day is the key independent variable. Nonetheless, a large volume and variety of experiments, often investigating other phenomena or as part of multivariable analyses, provide undeviating data to support the hypothesis 
that comfort decreases during the day and is exacerbated by contact lens wear.

The original empirical demonstration of decreasing comfort toward the end of the day appears to have been by Pritchard and Fonn. ${ }^{84}$ In their 1995 study, which sought to link lens dehydration with symptoms, 19 subjects rated dryness on visual analog scales during 7 hours' wear of three different hydrogel lenses. Dryness ratings rose consistently for all three lens types at 1, 3, and 7 hours after lens insertion. In a followup study, Fonn and colleagues sought to determine whether lens dehydration correlated with discomfort and dryness in two different lens types in 40 subjects, but this time they separated subjects into symptomatic and asymptomatic groups. ${ }^{102}$ They replicated the findings of increased dryness and decreased comfort over a 7-hour period of hydrogel lens wear in the asymptomatic group, but interestingly there was no significant variation over time in the symptomatic group. Others have confirmed the finding among hydrogel lens wearers. ${ }^{46,282,283}$

Fonn and Dumbleton ${ }^{47}$ published the first paper reporting reduced end-of-day comfort with SiHy lenses in a 7-hour nondispensing study of 20 asymptomatic and 19 asymptomatic wearers. Again, like the 1999 study from the same group, the reduced end-of-day comfort was isolated to the symptomatic group. The paper suggested that the degree to which comfort diminishes and dryness increases is very similar to that observed with hydrogel lenses. Many others have reported decreased comfort over the course of the day among SiHy wearers. ${ }^{61-65,276,284-291}$

Wear of daily disposable contact lenses is not protective from the effect of time of day on comfort and dryness. Walker and colleagues measured a drop in mean comfort across the day when comparing two different hydrogel daily disposable lenses in a 20-site study of 282 subjects. $^{292}$ Diec and colleagues $^{290}$ tracked decreasing comfort across the day for a series of hydrogel and SiHy lenses worn on a daily disposable basis. They found no difference between lenses but noticed decreased comfort across the course of the day.

While there may be differing degrees of discomfort between daily wearers and those who sleep in their contact lenses, extended or continuous wear also does not seem to prevent the phenomenon of decreased comfort and increased dryness toward the end of the day. In their 2005 study looking at wearers switched out of hydrogels and into silicone hydrogels, Chalmers and colleagues ${ }^{48}$ observed among the baseline data for those using hydrogel lenses on an extended basis, more than $50 \%$ greater frequency and severity of end-of-day dryness. Subbaraman and colleagues ${ }^{254}$ and Schafer and colleagues ${ }^{57}$ have made supporting observations with extended wear of silicone hydrogels. These consistent findings also seem to hold up across different ethnicities. Long and McNally ${ }^{286}$ studied symptoms in 88 Asian subjects that were switched from hydrogel lens wear to SiHy lens wear. Decreased comfort and increased dryness toward the end of day was evident both before and after switching lens type. Indeed, $84 \%$ of subjects reported end-of-day dryness either occasionally, frequently, or all the time with their habitual lenses.

Investigators have used variables based around wearing time both to demonstrate the consequence of end-of-day dryness and also as a measure of comfort for testing experimental hypotheses. ${ }^{32,49,63,65,285,292-294}$ As an example, Young and colleagues found mean comfortable wearing time in a group of UK subjects classified as having contact lens associated dry eye to be 9.1 hours compared with 12.4 hours in those without dry eye and, in a sample of North American wearers, 9.4 hours in the dry eye group compared with 12.1 hours in those without dry eye. ${ }^{63,65}$
The interaction between the lens and the lid wiper appears to play a significant role in end-of-day dryness and discomfort. ${ }^{295}$ Lens surface coefficient of friction appears to be correlated to overall lens comfort and in particular to end-ofday comfort (see previous section on friction and lubricity). ${ }^{116}$ Certainly the simple addition of lubricants yields immediate, if short-term, benefits in comfort. ${ }^{59}$ However, the mechanism by which coefficient of friction might be linked to end-of-day comfort is uncertain. Daily accumulation of lens surface buildup, or diminution of tear quality during the course of the day, may lead to higher lens surface friction later in the day. Alternatively, lens surface friction may remain relatively stable, although at a raised level during wear compared with the bare cornea, but the lid-wiper region becomes irritated or damaged with erosion of cells during the course of a day's wear as a result of the rubbing between the lid and the front surface of the lens and so becomes uncomfortable. Overnight, the affected epithelium repairs and, on awakening and reapplication of the contact lens, the cycle begins again.

To date, there are no records of measurement of lens surface friction changes over the course of a day. The more modest changes of comfort across days and weeks of wear as evidenced by the data on replacement schedules above compared with the dramatic change over the course of a day would argue more strongly for the latter hypothesis of "wear and tear and then repair" to explain end-of-day discomfort. One further piece of evidence relates to the fact that replacement of a lens during the middle of the day appears to have minimal impact on end-of-day comfort, ${ }^{296}$ suggesting that a fatigue-like response in one or more ocular tissues or stimulation of ocular surface nociceptors induced by the presence of the contact lens occurs. Without doubt, further research is needed to ascertain the true origin of end-of-day dryness with contact lens wear.

From a scientific standpoint, the hypothesis that contact lens comfort decreases toward the end of the day can never be tested in a controlled, randomized, masked study because normal subjects will always have awareness of the duration for which they have worn the lenses and naturally the sequence of such measures. Thus, while the various studies reported here may be controlled, randomized, and masked with respect to lenses, care systems, or some other independent variable, they are not with respect to time of day and daily duration of wear. Nonetheless, the wealth of reporting on the matter and the overwhelming consistency of the data lead us to identify this problem as one of the major, if not the leading, issue with contact lens wear today.

\section{Care Products and Packaging Solutions}

Compositions of Care Solutions. A contact lens care solution is composed of several important components, including preservatives (or biocides), surfactants, chelating agents, and buffering agents. All these components have different functions and are incorporated into a lens care system to provide adequate disinfection efficacy and enhanced comfort. The difference in clinical performance observed between various lens care solutions may be due to the differing components and concentrations in the care products and the manner in which these components interact with the lens material. An essential point to consider is that the care system can result in reduced comfort or enhanced comfort-two very different outcomes; one is likely caused by uptake and subsequent release of the components of the care system and the other by the adsorption of a comfort "additive" to the lens material from the care system.

Biocides. Various biocides are incorporated into lens care regimens at different concentrations and the effect of these 
biocides on subjective comfort and how these biocides interact with different lens materials has attracted significant interest.

Peroxide-Based Systems. Hydrogen peroxide-based solutions are used at a concentration of 3\% (30,000 ppm). The subjective sensitivity threshold for peroxide ranges between 50 and $300 \mathrm{ppm}$, and it is recommended that the solutions be neutralized to a concentration of less than $100 \mathrm{ppm}$. When present in high concentrations, residual peroxide can be toxic to the cornea and can cause discomfort/pain. ${ }^{297-300}$

Few studies have compared the effect of peroxide-based systems on subjective symptoms in a comparison with other care systems that are preserved with a different biocide. A randomized, single-masked, crossover design study evaluated the degree and frequency of corneal fluorescein staining and subjective responses in 85 hydrogel lens wearers following the use of PHMB-based (ReNu) and peroxide-based (AO Sept) systems for 1 month. ${ }^{301}$ It was found that the overall comfort and comfort in the evening were significantly better when the subjects used the peroxide-based system $(P=0.02$ for both occasions). Another study investigated the clinical and subjective performance of a peroxide-based lens care system (ClearCare) in comparison to a Polyquad/Aldox-based multipurpose solution (MPS; OPTI-FREE RepleniSH) when used with lotrafilcon B and senofilcon A SiHy lenses. ${ }^{285}$ This randomized, contralateral (lens type) and crossover (care system) study involved 24 participants and they found that the peroxidebased system resulted in longer reported comfortable wearing times than the MPS (10.93 vs. 9.84 hours; $P<0.01$ ). However, no significant difference was found between solutions in overall ratings of subjective comfort, or dryness. While these two studies taken in isolation would appear to suggest that peroxide-based systems are superior to preserved systems, there are multiple other components that differ between the products and this makes it impossible to support the fact that it is merely the biocide alone that resulted in the reported comfort differences.

PHMB-Based Versus Polyquad-Based Systems. Several studies have compared the effect of using a PHMB-based system when compared with a polyquaternium-based MPS. A multisite, 231-subject, double-masked, crossover study was performed to evaluate the subjective comfort and satisfaction and clinical signs with two MPSs used with alphafilcon A and etafilcon A lenses. ${ }^{302}$ Subjects used each of the two MPSs, Polyquad/Aldox-based (OPTI-FREE Express) and PHMB-based (ReNu MultiPlus), for 28 days and found that subjective ratings of comfort and satisfaction were in favor of the polyquad-based MPS. Epstein found that the users of the PHMB-preserved product reported decreased comfort over the course of the day. ${ }^{303}$ Interestingly, it was also reported that the PHMB-based system was also associated with a reduction in relative corneal sensitivity $(P=0.004)$. However, a subsequent letter to the editor questioned this latter finding. ${ }^{304} \mathrm{~A}$ randomized, controlled, and investigator-masked clinical study compared the clinical performance of a PHMB-based (MeniCare Soft) and Polyquad/Aldox-based (OPTI-FREE Express) MPS with two SiHy lenses (lotrafilcon A and galyfilcon A), ${ }^{305}$ and found no significant difference between the two solutions.

In another study, subjective symptoms and clinical signs of tolerability and comfort were compared in silicone and hydrogel lens wearers using a Polyquad/Aldox-based system (OPTI-FREE Express) and a PHMB-based system (ReNu MultiPlus). ${ }^{306}$ The participants who used the Polyquad/Aldox-based system reported greater comfort than the PHMB-based system. These results should be interpreted with caution because $65 \%$ of the subjects in this study used the Polyquad/Aldox-based system while only $28 \%$ used PHMB-based MPS prior to enrollment, and this could potentially create a bias toward their habitual care system.
Another study was conducted as a prospective, bilateral, clinical trial with a single-masked investigator, and randomized crossover design with four phases to assess the compatibility of a SiHy lens material with four different MPS (one based on Polyquad/Aldox: OPTI-FREE RepleniSH, and three based on PHMB: ReNu MultiPlus, Solo-Care Aqua, and MeniCare Soft). ${ }^{307}$ No difference was found in comfort between the four care systems. A recent study investigated the performance of two new MPSs (polyquaternium/alexidine-based Complete RevitaLens and polyquaternium/PHMB-based Biotrue) during a month of SiHy lens wear in neophyte volunteers. ${ }^{291}$ The investigators did not find statistically significant differences between the two systems.

Finally, a randomized, investigator-masked, crossover clinical trial including 31 subjects compared a Polyquad/Aldox (OPTI-FREE Express) solution to a PHMB-based solution (Complete Moisture Plus) in subjects wearing etafilcon A lenses. Each participant used the assigned care solution for 7 days, with a 1-day washout period, followed by subsequent use of the alternative solution. While interferometric differences in the prelens tear film thickness were observed (likely based on viscosity differences between the solutions), there was no overall difference in subject preference for a care solution, but "comfort" was the primary reason for a preference selection when asked their reason for preference. ${ }^{136}$

Long-Term Use of PHMB-Based Versus Polyquad-Based Systems. Long-term users of two different preservative systems were studied to investigate whether prolonged use of these systems was associated with an increase in the frequency of dry eye. ${ }^{308}$ Subjects were required to have consistently used a PHMB-based or polyquad-based solution for 2 years. This investigator-masked study, involving 89 FDA Group IV hydrogel or SiHy lens wearers, found that PHMB users reported significantly more grittiness or scratchiness (67\% vs. $44 \% ; P=$ 0.02). However, no significant differences between the two preservative system groups were noted for the range of other dry eye evaluations or the remaining clinical assessments. ${ }^{308}$

Studies Investigating Consumer Acceptance of MPS. One study evaluated comfort when switching to Polyquad/Aldox based MPS (OPTI-FREE RepleniSH) when compared with two different PHMB-preserved MPS. ${ }^{309}$ This multicenter, open-label study enrolled 109 contact lens wearers who were dispensed with the test solution in place of their habitual solution. Subjects assessed their experience with their habitual solution (baseline) and the test solution (day 30) using Likert-style questions. They reported that the Polyquad/Aldox MPS was associated with a statistically significant improvement in instillation comfort, end-of-day comfort, clear vision, and overall satisfaction. ${ }^{309}$ It is difficult to determine if a natural bias is introduced in studies such as these, as subjects given new solutions or lenses will often rate "new" products as being superior due to the mere fact that they are new, therefore, "they must be better."

In summary, although a few studies have shown that lens wearers using a care solution that is preserved with a specific biocide show better comfort than another product, it is important to note that a lens care solution is composed of many ingredients that may also impact subjective symptoms. Therefore, it cannot be concluded that a specific biocide alone will provide improved comfort.

Surfactants and Wetting Agents. In contact lens solutions, surfactants are used as detergents or cleaners, removing loose debris, microorganisms, and deposits by combining with these substances to form micelles, which are then removed during the rinsing procedure. Surfactants also play a role in enhancing the wettability of contact lenses, ${ }^{132,137,310}$ especially 
SiHy lenses, which are generally more hydrophobic than conventional hydrogel lens materials. ${ }^{311-313}$

The most common surfactants found in MPS are poloxamers (Pluronic F87, Pluronic F127, Pluronic 17R4) and poloxamines (Tetronic 1304, Tetronic 1107)..$^{314,315}$ HPMC, which has been used for many years in rigid gas-permeable care products for its lubricating, conditioning, and cushioning functions, has also been used in soft lens solutions as a wetting agent. ${ }^{316}$ It has been shown to be effective in controlling both symptoms and signs in patients with dry eye ${ }^{317}$ and to enhance tear film stability in lens wearers. ${ }^{134}$ A block copolymer (EOBO) containing poly(ethylene oxide) and poly(butylene oxide) has also been recently introduced and its ability to adsorb to SiHy materials has been confirmed using x-ray photoelectron spectroscopy and ultra-performance liquid chromatography. ${ }^{310}$

A randomized, controlled, double-masked, multicenter study involving 362 subjects at 19 investigational sites in the United States investigated the performance of two MPSs (tetronic-containing MPS [OPTI-FREE RepleniSH] and poloxamine-containing MPS [ReNu MultiPlus]) with habitual lenses. ${ }^{293}$ They found that the comfort and dryness mean scores were significantly better for the tetronic-containing MPS compared with the poloxamine-containing MPS at day 28 and the mean scores for scratchiness and burning were significantly lower at day 14. The improved performance of the MPS was attributed to the presence of wetting agents, including C9ED3A and propylene glycol in the tetronic-containing MPS. It was speculated that the presence of these novel wetting agents aids in cleaning, chelation, wetting, and lowering biocide lens uptake, especially in combination with Tetronic $1304 .^{293}$

The wetting effect of three different lens care solutions (two care solutions with wetting agents [OPTI-FREE RepleniSH and ReNu MultiPlus] and one solution without any wetting agent [ClearCare]) on blink rate, dryness symptoms, and vision performance on 65 habitual lens wearers was studied. ${ }^{318}$ They found that solutions with wetting agents led to significantly fewer eye blinks and better ocular comfort for contact lens wearers. Moreover, the presence of wetting agents in lens care solutions also resulted in better visual performance when compared with wearing daily disposable contact lenses.

The effect of an EO-BO containing MPS (OPTI-FREE PureMoist) was compared with a MPS containing a conventional surfactant-containing MPS (ReNu Fresh) with SiHy and HEMA-based lenses. ${ }^{319}$ It was a multicenter (30-site) study involving 573 participants over several visits. The patients found that their "lenses felt moist" at day 90 when using the MPS containing the novel surfactant $(P \leq 0.02)$ and the "lens acceptability at day 90 " was better when the MPS with the novel surfactant was used $(P \leq 0.03) .{ }^{319}$ Another study that recruited over 3000 patients from 313 ophthalmologic practices in France to participate in a 1-month prospective observational clinical study found that replacing etafilcon A lenses once every 2 weeks combined with an MPS incorporating ingredients designed for lens conditioning contributed to significant improvements in lens wearing comfort. ${ }^{275}$ Another study showed that HPMC incorporated in an MPS could form a thicker, longer-lasting layer of fluid on the hydrogel lens, leading to improvements in tear function in contact lens wearers. ${ }^{134}$

In summary, based on these studies, it appears that many wetting agents and/or surfactants that can remain on the lens material can improve subjective symptoms in contact lens wearers, possibly by improving the lens hydrophilicity and also by making the lenses feel "moist." However, the long-term efficacy of these surfactant-containing solutions in patients who are dry-eyed and need contact lenses warrants further investigation.
Chelating Agents. Chelating agents are added to lens care regimens to act synergistically with other agents to improve disinfection efficacy and to aid in removal of tear film components. ${ }^{314}$ The common chelating agents found in lens care regimens include EDTA, citrate, and hydroxyalkylphosphonate. ${ }^{315}$ Studies published to date have compared the clinical performance of a specific care regimen in comparison with only citrate-containing regimens. ${ }^{252,320,321}$ A multicenter, investigator-masked, randomized study investigated the effect of two citrate-containing regimens on subjective comfort and deposition on a FDA Group IV lens material and compared that with a noncitrate containing MPS. ${ }^{320}$ Significant differences favoring the citrate-based regimens were observed in ocular awareness, lens awareness, visual clarity, end-of-day comfort, and end-of-day dryness. ${ }^{320}$ These findings were consistent with that of another study, where it was found that the use of a dedicated daily cleaner in conjunction with a citrate-containing system can provide patients with more comfortable and cleaner lenses. ${ }^{252}$ Another study that compared comfort when using a citrate-containing MPS versus an MPS containing HPMC found no significant difference in comfort between the two solutions. ${ }^{321}$

In summary, all the above-mentioned studies compared a few lens care solutions and attributed any increased performance to the presence of a specific component. However, this is not possible to prove, as other formulation differences between the products may also have contributed to the perceived differences in comfort. In order to specifically determine the association between lens care solution and discomfort and dryness, two studies have conducted extensive regression analysis. In the first study, ${ }^{32}$ the relationship between contact lens characteristics, hydrogel lens materials, care solutions, and patient-related factors and dry eye status in contact lens wearers was assessed retrospectively in 360 contact lens wearers. Interestingly, there was no significant association between contact lens-related dry eye and contact lens care solutions, when grouped either by preservative type or by product brand (both $P=0.99$ ). ${ }^{32}$ Another more recent study examined the factors associated with contact lensrelated dryness symptoms in soft contact lens patients. ${ }^{63}$ Soft contact lens wearers $(n=932)$ from 12 clinical sites were examined and they found that neither the lens material nor the lens care systems were specifically related to contact lensrelated dry eye status.

These two studies suggest that contact lens-related dryness is associated with a diverse range of underlying causes and that lens care product is not a significant factor. It should be noted that both these studies derived data from retrospective studies by pooling data from multiple studies and/or sites and conducted advanced statistical analysis to determine the association between dry eye symptoms and the lens care regimen. Nevertheless, it is important to identify how different components in a lens care solution interact with contact lens materials and if this could have an impact on the physiological and subjective performance of contact lenses.

Interaction of Contact Lenses With MPS. Silicone hydrogel materials are hydrophobic and these materials may exhibit higher attraction for certain hydrophobic/lipophilic entities, such as tear lipids ${ }^{322}$ and nonpolar active agents found in certain MPS products. ${ }^{323,324}$ When a MPS interacts with a contact lens, any of the components found in the solution can be adsorbed onto the surface or absorbed into the bulk of the lens material. ${ }^{310,323,325,326}$ Preservative uptake from lens care solutions to soft lens materials is influenced by several properties of the lens, including EWC, ionicity, and hydrophobicity. ${ }^{323,324,327}$ These adsorbed components may potentially cause discomfort to contact lens wearers. 
The biocide uptake into and onto various contact lenses and its subsequent influence on clinical signs and symptoms were investigated in several studies. ${ }^{326,328-331}$ The physiological and subjective responses of subjects wearing balafilcon A silicone hydrogels and the ocular response to use of a lens care product containing Polyquad/Aldox and another containing PHMB was reported by Jones and colleagues. ${ }^{328}$ The PHMB-based lens care product was associated with increased corneal and conjunctival staining and more stinging or burning on lens insertion compared with the product containing Polyquad/ Aldox. ${ }^{328}$ However, the investigators were not able to relate the degree of staining with the reported symptoms. This is in contrast to two more recent publications, in which increased amounts of corneal staining led to reduced subjective comfort. ${ }^{329,332}$

A series of pilot studies was conducted over 11 months to assess combinations of three different hydrogel lenses (FDA Group II [alphafilcon A], Group IV [etafilcon A] and one SiHy [lotrafilcon A]) and four MPSs. ${ }^{331}$ New lenses were soaked overnight in one of four MPSs and were fitted on subjects who rated comfort and ocular symptoms. Corneal staining was evaluated at baseline and after lens removal. The investigators found corneal staining to be most frequent when PHMBpreserved solutions were used with Group II lenses. Instead, with the polyquad-based system, the extent of staining was low with all the lenses tested. They also found that when PHMBbased products were used with the FDA Group II material, corneal staining was evident after 1 to 4 hours of wear. However, they did not see any association between significant symptoms and the extent of staining. ${ }^{331}$

Another study investigated the physiological and subjective responses of the short-term use of various lens care products with two SiHy contact lenses (lotrafilcon B or galyfilcon A) and examined whether changes to the surface of lenses was correlated with the responses. ${ }^{326}$ Both these lens types were presoaked for 1 week in Polyquad/Aldox-based (OPTI-FREE Express) or PHMB-based (Aquify) solution and participants wore them for 6 hours. It was found that lotrafilcon B lenses soaked in PHMB caused a decrease in comfort, an increase in burning/stinging after 1 hour of wear, and an increase in lens awareness on lens insertion. When lotrafilcon B lenses were soaked in Polyquad/Aldox, they found an increase in burning/ stinging after 1 and 6 hours. ${ }^{326}$ The investigators concluded that release of various components of MPS from contact lenses can have a significant influence on corneal staining and comfort responses during wear.

In summary, the results from these studies show that contact lenses interact differently with MPS depending upon their polymeric makeup. These results also show that the uptake and subsequent release of components by soft contact lenses can affect corneal staining and subjective comfort.

In conclusion, while retrospective studies suggest that the lens care product is not associated with the contact lensrelated dryness and discomfort, the importance of contact lens care solutions in overall lens wear cannot be discounted and a recent publication has shown that subjective satisfaction, particularly in symptomatic wearers, can be influenced by the combination of lens and solution prescribed ${ }^{333}$ It is critical to note that a lens care solution is composed of several components. Therefore, it would be erroneous to conclude that any individual component in a care solution will have a direct impact on subjective symptoms. Based on the evidence to date, it appears that incorporation of surfactants or wetting agents into lens care products may improve subjective comfort, possibly by improving the hydrophilicity of the lens material. However, it is difficult to isolate a specific component in a lens care product and correlate that factor with improved subjective symptom.
There is adequate evidence that suggests different lens care solutions interact differently with various contact lens materials and this depends on the properties of both the contact lens material and lens care solution. Thus, the mechanisms contributing to symptomatology during lens wear may vary based on how the components in a lens care solution interact with the lens material.

\section{Physical Properties of Care Solutions}

Soft contact lens care solutions are made of a wide range of components, as described in the previous section. The combination and concentration of these agents will have a significant impact on the physical properties of the solution and this could potentially influence patient comfort. The following section provides a brief overview of various physical properties of contact lens care solutions and whether these properties may have an impact on contact lens comfort.

pH. The $\mathrm{pH}$ of human tears ranges between 6.6 and $7.8,{ }^{334}$ and the human eye is capable of tolerating $\mathrm{pH}$ values in the range of 6.2 to 9.0 at $0.2 \mathrm{M}$ strength. ${ }^{335}$ When the $\mathrm{pH}$ of the contact lens solution falls outside this range, patients complain of ocular discomfort and stinging. ${ }^{336-338}$ Buffering agents used in soft contact lens solutions directly affect their $\mathrm{pH}$, and it is possible that the type of buffer used in a particular solution could also affect subsequent patient comfort.

A study that investigated the $\mathrm{pH}$ of 10 different contact lens care solutions showed that most solutions (except nonneutralized peroxide systems) had $\mathrm{pH}$ values that were close to neutral and fell within the reported tolerable $\mathrm{pH}$ range for the ocular surface. ${ }^{339}$ The large difference in the $\mathrm{pH}$ of peroxidebased solutions before neutralization is the principal reason for burning, stinging, and epithelial cell damage seen in patients who mistakenly insert the nonneutralized solutions directly onto the ocular surface. ${ }^{299}$

Viscosity. Viscosity of a solution has the potential to influence patient comfort upon lens insertion or at the end of the day, through interactions between the solution, the lens, and the patient's tear film. Viscosity of water is $1.0 \mathrm{cP}$ and that of the tear film ranges between approximately 5.0 and $1.5 \mathrm{cP}$ at $25^{\circ} \mathrm{C}$ for normal patients. ${ }^{340,341}$

A study that investigated the viscosity of various lens care solutions found that all the solutions had viscosity values that ranged between 0.96 and $1.26 \mathrm{cP}$, but some go as high as 3 cP. $^{339}$ Several studies have determined the impact of HPMCcontaining solutions on patient comfort. ${ }^{134,136,342}$ One study investigated the physical properties of multipurpose contact lens solutions with and without the addition of HPMC, and also determined if there are significant differences in the tear physiology of two groups of patients wearing soft contact lenses soaked in HPMC and non-HPMC solutions. ${ }^{134}$ This study showed that the prelens tear thinning time was longer and the prelens tear film structure was improved with use of the HPMC-containing solution. ${ }^{134}$ Another clinical trial that compared the prelens tear film thickness of etafilcon lens wearers showed that the patients who used the HPMC-containing solution showed a greater prelens tear film thickness (3.02 \pm $1.07 \mu \mathrm{m})$ when compared with those that used a non-HPMC containing solution $(2.72 \pm 0.86 \mu \mathrm{m}) .{ }^{124}$ This study also showed no statistical difference in study subjects' preference for either solution, but nearly every subject (90.3\%) suggested "comfort" as their reason for preference. ${ }^{124}$ In summary, it appears that lens care solutions that incorporate viscosityenhancing agents can create a thicker and longer-lasting layer of fluid on hydrogel lens and this can potentially lead to improvements in tear function in contact lens wearers. However, if the viscosity is too high, then potential blurring effects may mitigate these comfort advantages. 
Osmolality. Osmolality of contact lens solutions could play a role in patient comfort, as studies have demonstrated that tear film osmolality plays a significant role in the discomfort reported by dry eye patients. ${ }^{343-346}$ A study that investigated the osmolality of 10 different contact lens care solutions showed that the osmolality values fell between 275 and 310 $\mathrm{mOsm} / \mathrm{kg},{ }^{339}$ indicating that the majority of soft contact lens solutions are hypo-osmotic compared with human tears. To our knowledge, no published studies have been conducted to investigate the relationship between solution osmolality and contact lens comfort.

Surface Tension. The surface tension of pure water is approximately $72 \mathrm{mN} / \mathrm{m}$ and human tears have a surface tension value in the range of 40 to $46 \mathrm{mN} / \mathrm{m} \cdot{ }^{340,347}$ In a contact lens care solution, the presence/absence and type/number of surfactants will have a substantial impact on the surface tension of the solution. A study that investigated the surface tension of various care solutions showed that most multipurpose solutions have surface tension values that ranged between 29 and $40 \mathrm{mN} / \mathrm{m}^{339}$ Among all the care solutions, the ones that did not incorporate surfactants (for example, peroxide-based systems and saline) had surface tension values that were close to that of water, whereas the solutions that had one or more surfactants had surface tension values that were closer to that of human tears. To our knowledge, no published studies have been specifically conducted to investigate the relationship between surface tension and comfort.

In summary, contact lens care solutions differ in certain physical properties and, by design, most care solutions fall within acceptable limits of ocular physiological tolerance. When properties of these solutions do not fall within the acceptable limits, clinically, this could result in burning, stinging, and epithelial cell damage. Minor shifts in the values may have the potential to influence patient comfort initially and/or at the end of the day. To date, very little has been published directly investigating the relationship between physical properties of lens care solutions with contact lens symptoms and this warrants further investigation.

\section{Rewetting Drops}

Contact lens wearers use rewetting drops for many reasons, including managing contact lens dry eye, lens dehydration and its associated dryness, general ocular lubrication, acting as a mechanical buffer between the lens and cornea, and lens surface rewetting and cleaning. Numerous formulations of lubricating eye drops exist and contain a wide variety of ingredients including cellulose derivatives, oil-based emulsions, paraffin, polyvinyl alcohol, polyacrylic acid, Polyvinylpyrrolidone, glycerin, HA, hydroxypropyl guar, polyethylene glycol, and propylene glycol.

It has been reported that $47 \%$ of contact lens wearers use rewetting drops, but that they only provide moderate and nonsustained relief from symptoms of discomfort, in addition to inconvenience with the need for repeat instillation. ${ }^{280}$ There is the suggestion that a regimen of more than one type of lubricating eye drop may be needed for symptomatic contact lens wearers due to the multifactorial nature of CLD. ${ }^{348}$ If used in a proactive manner, the same eye drop has been shown to produce greater symptom relief than its use in a reactive manner. ${ }^{349}$ While there are a number of studies that demonstrate some level of symptom relief for contact lens wearers, there appears to be relatively little advantage with the use of rewetting drops or ocular lubricants compared to the use of saline. ${ }^{59,254,350-353}$

One study has shown that the use of a lubricant eye drop containing hydroxypropyl guar, propylene glycol, and polyethylene glycol (Systane) twice daily (pre- and post-contact lens wear) resulted in an increase in comfortable wear time and improved other subjective assessments of lens wear acceptability in symptomatic hydrogel lens wearers, compared with the use of a saline control drop (Feng Y, et al. IOVS 2006; 47:ARVO E-Abstract 2381).

\section{Composition of Packaging Solutions}

In an attempt to alleviate symptoms of dryness and discomfort, a number of manufacturers incorporate a variety of agents into both the lens material and also, more recently, the packaging solutions that are used to ship contact lenses. These so-called "blister-pack solutions (BPS)" now commonly include incorporation of water-soluble polymers, surfactants, and often unnamed "wetting agents" that have been previously described in this report. ${ }^{153,354-359}$ The alterations made to the BPS are to aid in preventing the lenses from sticking to the blister pack, enhance lens wettability, and improve initial comfort of the lenses after application to the eye. ${ }^{354}$

One of the earliest published studies to suggest that adhering surfactants to lens materials may enhance in-eye comfort was that by Tonge and colleagues, ${ }^{137,354}$ who showed that soaking etafilcon A lenses in a solution containing poloxamine 1107 improved in-eye comfort and that the surfactant was retained for several hours after wear. To date, while several studies have examined comfort between lens materials with and without wetting agents (as previously described in this review) and studies have shown that contact angle wettability is raised in some materials once the BPS is removed, ${ }^{312,356}$ no published study has systematically examined whether the modified BPS has a direct impact on contact lens comfort. However, given the growth in the number of lens materials that are shipped in BPS that contain surface-active agents $^{357}$ it would seem likely that manufacturers have determined their positive impact in in-house nonpublished studies.

\section{Future Directions}

Careful review of this report points toward several areas for future research that would enhance our understanding of CLD and the key factors associated with materials, design, and care systems.

One area that continues to be a frustration is the lack of association between in vitro data and their ability to predict in vivo performance. The industry would be well served by the development of in vitro methodologies that help to predict oneye performance. An area that appears to show the most promise relates to the results from coefficient of friction evaluations. However, this field is in its relative infancy for contact lens materials and the development of methods that are more meaningful to on-eye comfort require substantial work. Current assessment methods vary widely, resulting in wide variation in the data obtained. International standardization of laboratory measurements such as frictional testing of hydrogels, as well as those for others such as contact angle and dehydration, would be a welcome addition to the industry. For frictional measurements, the relative importance of methodology, type of friction, and how these values relate to clinical observations such as lid wiper epitheliopathy, lid-parallel conjunctival folds, corneal and conjunctival staining, and ineye wettability, are all areas worthy of future investigation. Other related areas that need substantial development relate to improved methods for determining in-eye wettability and investigation of the tear film in the vicinity of the lid wiper area and how these factors relate to comfort.

There is no doubt that contact lens materials change their hydration after being placed onto the ocular surface. It would 
be expected that a change in bulk surface hydration will impact wettability and friction. However, bulk dehydration shows only a tenuous relationship with comfort for most materials, but the development of improved methods to investigate surface dehydration (and impact on comfort) are more important than ever due to new materials that exhibit differing bulk and surface characteristics. When undertaking dehydration studies, factors such as the time intervals for assessment, standard procedures for sample handling, and eventual reinsertion in the ocular surface for subsequent measures, and control of environmental conditions and time of day all need standardization and agreement.

Much of the published clinical work in relation to soft lens material, design or solution properties has been poorly conducted, with inappropriate or missing controls, making conclusions regarding their impact on discomfort difficult. Future work investigating the impact of various characteristics must be conducted using well-controlled, randomized, crossover studies in which all variables (replacement period, solution system, wearing time, etc.) are considered. This area requires some fundamental studies in which the isolation of a single change in a material, design, or solution characteristic is investigated. This work can only be conducted with the close cooperation of industry since this cannot be undertaken using only commercially available products. Areas of investigation of particular note relate to comparisons of some of the newer hydrogel-based materials against modern silicone hydrogels, comparisons between materials that "release" components into the tear film versus the base material without the release agent, and silicone hydrogels with standard or base surface wettability versus those with enhanced hydrogel-type coatings.

When it comes to bulk material properties, the trend thus far has been toward a modulus low enough to maximize on-eye comfort while balancing handling, durability, and rightness of fit. However, the conventional tensile modulus test involves unidirectional static loading; therefore, dynamic mechanical testing may be more appropriate, given the cyclic dynamic motion of eyelid movement coupled with the elastic and viscous flow characteristics of hydrogel materials (the cornea is also viscoelastic). For example, dynamic mechanical testing of silicone hydrogels demonstrates a characteristic rise in elastic modulus or shear-dependent elastic response that is typically not present in conventional hydrogels with similar EWC. There is the suggestion that, in order to improve comfort of current SiHy lenses, this elastic component should be similar to that for conventional hydrogels. However, this work has not been conducted in a systematic manner.

With regard to rigid lenses, increased interest in the use of RGP scleral lenses points toward a substantial number of potential studies in this area, with specific data being needed on comfort changes over the course of the day and comparative studies against corneal rigid lenses in nonpathological corneas. Large diameter RGP lenses offer an opportunity to provide a test platform with no bulk hydration changes, slower deposition, limited lens movement and minimal lid-lens edge interaction. Thus, studies comparing RGP scleral designs against both corneal rigid lenses and soft lenses may be of value. Of specific interest in the area of both rigid and soft lens design, methods to assess the impact of lens "edge" design oneye must be developed, such that differing designs in identical materials can be compared. Methods to investigate tear replenishment and expulsion from beneath lenses must also be developed to aid us in understanding their impact on factors such as end-of-day comfort and inflammatory responses.

Further work to better understand the reasons behind the success of frequent replacement lenses is also needed, particularly with new materials. What is the optimal period of replacement for certain materials, and what are the methods used to determine this? How widely does this differ for different patients? What are the factors associated with the optimal replacement period? Is it due to changes in the material itself, the accumulation of certain tear film components, or those from the care system? What changes occur over time (over the day and over the lifetime of the lens) to both the ocular tissues in contact with the lens and the material itself? On a related note, studies to better understand the accumulation of tear film components remain to be undertaken. In particular, a better understanding of the impact of denatured proteins (other than lysozyme), lipid breakdown products and the deposition of many other tear film components are required.

There also remain large gaps in our knowledge of the role of care systems, packaging solutions and "comfort" drops on CLD. As with material-based studies, potentially valuable investigations in which systematic changes in various components are evaluated have yet to be undertaken. The short- and long-term impact of the uptake and release of lens care components and how they affect comfort are areas of future interest. Should care systems remove all tear film constituents that are deposited onto materials, or should they be designed to leave in place certain components that may help "biocompatibility"? If some components should be left in place, which ones and how much is "enough"? Finally, how effective is the delivery of wetting agents from the materials in reducing CLD and which agents are the most efficacious-and for how long?

\section{Summary}

In summary, a thorough review of the literature shows that there are surprisingly few proven links between CLD and factors related to the contact lens material, design, and care system. However, clinical acumen (in addition to recent studies $^{277,333}$ ) demonstrates that, in contact lens wearers who exhibit unacceptable comfort, making changes to the lens material, design, care system, and replacement schedule can improve comfort. It is also pertinent to consider, as pointed out in this review, the limitations of laboratory and academic studies, which might miss relevant variables present in the "real world." Conclusions derived from well-conducted, well-controlled groups of subjects in a formal clinical trial might not be transferable to the thousands of patients that ultimately use the products, subjected to issues such as noncompliance, that may directly impact evaluation of comfort.

Much work remains to unravel the complexities of CLD. It is clear that a number of fundamental studies must be undertaken if an increased understanding of the role of materials, design and care regime in contact lens dryness is to occur. This will require substantial intellectual input and funding from both industry and academia alike.

\section{Acknowledgments}

Disclosure: Each workshop participant's disclosure data can be found in the Appendix of the Introduction.

\section{References}

1. Morgan PB, Woods C, Tranoudis I, et al. International contact lens prescribing in 2012. Contact Lens Spect. 2013;28:31-44.

2. Wichterle $\mathrm{O}$, Lim D. Hydrophilic gels for biological use. Nature. 1960;185:117-118.

3. Wichterle O. The Beginning of the Soft Lens. In: Ruben M, ed. Soft Contact Lenses, Clinical and Applied Technology. London, United Kingdom: Bailliére Tindall; 1978:3-5. 
4. Tighe B. Contact lens materials. In: Phillips A, Speedwell L, eds. Contact Lenses. Edinburgh: Butterworth-Heinemann; 2006:59-78.

5. Martin DK. Water transport in dehydrating hydrogel contact lenses: implications for corneal desiccation.J Biomed Mater Res. 1995;29:857-865.

6. McConville P, Pope JM. Diffusion limited evaporation rates in hydrogel contact lenses. CLAO J. 2001;27:186-191.

7. Orsborn GN, Zantos SG. Corneal desiccation staining with thin high water content contact lenses. CLAO J. 1988;4:8185.

8. Nichols JJ, Sinnott LT. Tear film, contact lens, and patientrelated factors associated with contact lens-related dry eye. Invest Ophthalmol Vis Sci. 2006;47:1319-1328.

9. Port M, Bowers R. A new hydrogel material for contact lenses. J Brit Contact Lens Assoc. 1993;16:154.

10. Young G, Bowers R, Hall B, Port M. Six month clinical evaluation of a biomimetic hydrogel contact lens. CLAO J. 1997; 23:226-236.

11. Young G, Bowers R, Hall B, Port M. Clinical comparison of Omafilcon A with four control materials. CLAO J. 1997;23: 249-258.

12. Lebow $\mathrm{K}$, Bridgewater B. A three-month comparative dailywear study of two high water content soft lenses. Int Contact Lens Clin. 1997;24:198-206.

13. Hall B, Jones S, Young G, Coleman S. The on-eye dehydration of proclear compatibles lenses. CLAO J. 1999;25:233-237.

14. Goda T, Shimizu T, Ishihara K. Bioinspired biomaterials for soft contact lenses. In: Chirila $\mathrm{T}$, ed. Biomaterials and Regenerative Medicine in Ophthalmology. Boca Raton, FL: CRC Press; 2010:263-279.

15. Quesnel NM, Giasson CJ. On-eye dehydration of Proclear, Resolution 55G and Acuvue contact lenses. Cont Lens Anterior Eye. 2001;24:88-93.

16. Cho P, Ng V. Clinical performances of two disposable soft contact lenses of different materials on Hong Kong-Chinese. Cont Lens Anterior Eye. 2000;23:53-60.

17. Bursinger U. A new material on the block of frequent replacement lenses. Contact Lens Spect. 2000;15:49-51.

18. Riley C, Chalmers RL, Pence N. The impact of lens choice in the relief of contact lens related symptoms and ocular surface findings. Cont Lens Anterior Eye. 2005;28:13-19.

19. Dumbleton K, Jones L. Extended and continuous wear. In: Bennett E, Henry V, eds. Clinical Manual of Contact Lenses. Philadelphia, PA: Lippincott Williams and Wilkins; 2008:410443.

20. Tighe B. Silicone hydrogel materials-how do they work? In: Sweeney D, ed. Silicone Hydrogels: The Rebirth of Continuous Wear Contact Lenses. Oxford, UK: ButterworthHeinemann; 2000:1-21.

21. Jones L, Dumbleton K. Soft lens extended wear and complications. In: Hom MM, Bruce A, eds. Manual of Contact Lens Prescribing and Fitting. Oxford, UK: Butterworth-Heinemann; 2006:393-441.

22. French K, Jones L. A decade with silicone hydrogels: Part 1. Optom Today. 2008;48:42-46.

23. French K, Jones L. A decade with silicone hydrogels: Part 2. Optom Today. 2008;48:38-43.

24. Nicolson P, Vogt J. Contact lenses: silicone hydrogels. In: Encyclopedia of Biomaterials and Biomedical Engineering. Boca Raton, FL: Taylor \& Francis; 2013:1-14.

25. Tighe BJ. A decade of silicone hydrogel development: surface properties, mechanical properties, and ocular compatibility. Eye Contact Lens. 2013;39:4-12.

26. Tighe B. Extended wear contact lenses. In: Chirila T, ed. Biomaterials and Regenerative Medicine in Ophthalmology. Boca Raton, FL: CRC Press; 2010:304-336.
27. Tighe B, Bharma T, Campbell D, Carnell S, Eden R. A decade of silicone hydrogel development: how has clinical experience informed material design? Cont Lens Anterior Eye. 2012;35(suppl 1):e14.

28. Nicolson PC, Vogt J. Soft contact lens polymers: an evolution. Biomaterials. 2001;22:3273-3283.

29. Kunzler JF. Silicone hydrogels for contact lens application. Trends Polym Sci. 1996;4:52-59.

30. Stone R. Why contact lens groups? Contact Lens Spect. 1988; 3:38-41.

31. Hutter JC, Green JA, Eydelman MB. Proposed silicone hydrogel contact lens grouping system for lens care product compatibility testing. Eye Contact Lens. 2012;38:358-362.

32. Ramamoorthy P, Sinnott LT, Nichols JJ. Treatment, material, care, and patient-related factors in contact lens-related dry eye. Optom Vis Sci. 2008;85:764-772.

33. Wilson G, Schwallie JD, Bauman RE. Comparison by contact lens cytology and clinical tests of three contact lens types. Optom Vis Sci. 1998;75:323-329.

34. Guillon M, McGrogan L, Guillon JP, Styles E, Maissa C. Effect of material ionicity on the performance of daily disposable contact lenses. Cont Lens Anterior Eye. 1997;20:3-8.

35. Efron N, Brennan NA, Currie JM, Fitzgerald JP, Hughes MT. Determinants of the initial comfort of hydrogel contact lenses. Am J Optom Physiol Opt. 1986;63:819-823.

36. Young G. Evaluation of soft contact lens fitting characteristics. Optom Vis Sci. 1996;73:247-254.

37. Dumbleton KA, Woods CA, Jones LW, Fonn D. Comfort and adaptation to silicone hydrogel lenses for daily wear. Eye Contact Lens. 2008;34:215-223.

38. Millodot M. Effect of soft lenses on corneal sensitivity. Acta Ophthalmol (Copenh). 1974;52:603-608.

39. Millodot M. Effect of hard contact lenses on corneal sensitivity and thickness. Acta Ophthalmol (Copenh). 1975;53:576-584.

40. Millodot M. Effect of long-term wear of hard contact lenses on corneal sensitivity. Arch Ophthalmol. 1978;96:12251227.

41. Millodot M, O'Leary DJ. Effect of oxygen deprivation on corneal sensitivity. Acta Ophthalmol (Copenh). 1980;58: 434-439.

42. Millodot M. A review of research on the sensitivity of the cornea. Ophthalmic Physiol Opt. 1984;4:305-318.

43. Bennett ES, Smythe J, Henry VA, et al. Effect of topical anesthetic use on initial patient satisfaction and overall success with rigid gas permeable contact lenses. Optom Vis Sci. 1998;75:800-805.

44. Brennan NA, Coles ML, Comstock TL, Levy B. A 1-year prospective clinical trial of balafilcon a (PureVision) siliconehydrogel contact lenses used on a 30-day continuous wear schedule. Ophthalmology. 2002;109:1172-1177.

45. Malet F, Pagot R, Peyre C, et al. Subjective experience with high-oxygen and low-oxygen permeable soft contact lenses in France. Eye Contact Lens. 2003;29:55-59.

46. Morgan PB, Efron N. Hydrogel contact lens ageing. CLAO J. 2000;26:85-90.

47. Fonn D, Dumbleton K. Dryness and discomfort with silicone hydrogel contact lenses. Eye Contact Lens. 2003;29(suppl 1): S101-S104; discussion S115-S118, S192-S194.

48. Chalmers RL, Dillehay S, Long B, et al. Impact of previous extended and daily wear schedules on signs and symptoms with high Dk lotrafilcon A lenses. Optom Vis Sci. 2005;82: 549-554.

49. Riley C, Young G, Chalmers R. Prevalence of ocular surface symptoms, signs, and uncomfortable hours of wear in contact lens wearers: the effect of refitting with daily-wear 
silicone hydrogel lenses (senofilcon a). Eye Contact Lens. 2006;32:281-286.

50. Santodomingo-Rubido J, Wolffsohn JS, Gilmartin B. Changes in ocular physiology, tear film characteristics, and symptomatology with 18 months silicone hydrogel contact lens wear. Optom Vis Sci. 2006;83:73-81.

51. Dumbleton K, Keir N, Moezzi A, Feng Y, Jones L, Fonn D. Objective and subjective responses in patients refitted to daily-wear silicone hydrogel contact lenses. Optom Vis Sci. 2006;83:758-768.

52. Young G, Riley CM, Chalmers RL, Hunt C. Hydrogel lens comfort in challenging environments and the effect of refitting with silicone hydrogel lenses. Optom Vis Sci. 2007; 84:302-308.

53. Dillehay SM, Miller MB. Performance of Lotrafilcon B silicone hydrogel contact lenses in experienced low-Dk/t daily lens wearers. Eye Contact Lens. 2007;33(6 pt 1):272-277.

54. Cheung SW, Cho P, Chan B, Choy C, Ng V. A comparative study of biweekly disposable contact lenses: silicone hydrogel versus hydrogel. Clin Exp Optom. 2007;90:124131.

55. Bergenske $\mathrm{P}$, Long B, Dillehay S, et al. Long-term clinical results: 3 years of up to 30-night continuous wear of lotrafilcon A silicone hydrogel and daily wear of low-Dk/t hydrogel lenses. Eye Contact Lens. 2007;33:74-80.

56. Brennan NA, Coles ML, Connor HR, McIlroy RG. A 12-month prospective clinical trial of comfilcon A silicone-hydrogel contact lenses worn on a 30-day continuous wear basis. Cont Lens Anterior Eye 2007;30:108-118.

57. Schafer J, Mitchell GL, Chalmers RL, et al. The stability of dryness symptoms after refitting with silicone hydrogel contact lenses over 3 years. Eye Contact Lens. 2007;33: 247-252.

58. Ousler GW III, Anderson RT, Osborn KE. The effect of senofilcon A contact lenses compared to habitual contact lenses on ocular discomfort during exposure to a controlled adverse environment. Curr Med Res Opin. 2008;24:335-341.

59. Ozkan J, Papas E. Lubricant effects on low Dk and silicone hydrogel lens comfort. Optom Vis Sci. 2008;85:773-777.

60. Chalmers RL, Hunt C, Hickson-Curran S, Young G. Struggle with hydrogel CL wear increases with age in young adults. Cont Lens Anterior Eye. 2009;32:113-119.

61. Long B, Schweizer H, Bleshoy H, Zeri F. Expanding your use of silicone hydrogel contact lenses: using lotrafilcon A for daily wear. Eye Contact Lens. 2009;35:59-64.

62. Santodomingo-Rubido J, Barrado-Navascues E, Rubido-Crespo MJ. Ocular surface comfort during the day assessed by instant reporting in different types of contact and non-contact lens wearers. Eye Contact Lens. 2010;36:96-100.

63. Young G, Chalmers RL, Napier L, Hunt C, Kern J. Characterizing contact lens-related dryness symptoms in a cross-section of UK soft lens wearers. Cont Lens Anterior Eye. 2011;34:64-70.

64. Maissa C, Guillon M, Garofalo RJ. Contact lens-induced circumlimbal staining in silicone hydrogel contact lenses worn on a daily wear basis. Eye Contact Lens. 2012;38:1626.

65. Young G, Chalmers R, Napier L, Kern J, Hunt C, Dumbleton K. Soft contact lens-related dryness with and without clinical signs. Optom Vis Sci. 2012;89:1125-1132.

66. Martin R, de Juan V, Rodriguez G, Martin S, Fonseca S. Initial comfort of lotrafilcon A silicone hydrogel contact lenses versus etafilcon A contact lenses for extended wear. Cont Lens Anterior Eye. 2007;30:23-28.

67. Brennan N. Beyond flux: total corneal oxygen consumption as an index of corneal oxygenation during contact lens wear. Optom Vis Sci. 2005;82:467-472.
68. Morgan PB, Efron N. Comparative clinical performance of two silicone hydrogel contact lenses for continuous wear. Clin Exp Optom. 2002;85:183-192.

69. French K. Contact lens material properties part 2: mechanical behaviour and modulus. Optician. 2005;230:29-34.

70. Horst CR, Brodland B, Jones LW, Brodland GW. Measuring the modulus of silicone hydrogel contact lenses. Optom Vis Sci. 2012;89:1468-1476.

71. Jones L, Subbaraman LN, Rogers R, Dumbleton K. Surface treatment, wetting and modulus of silicone hydrogels. Optician. 2006;232:28-34.

72. Tranoudis I, Efron N. Tensile properties of soft contact lens materials. Cont Lens Anterior Eye. 2004;27:177-191.

73. Dumbleton K. Adverse events with silicone hydrogel continuous wear. Cont Lens Anterior Eye. 2002;25:137-146.

74. Dumbleton K. Noninflammatory silicone hydrogel contact lens complications. Eye Contact Lens. 2003;29(suppl 1): S186-S189; discussion S190-S194.

75. Lin MC, Yeh TN. Mechanical complications induced by silicone hydrogel contact lenses. Eye Contact Lens. 2013;39: 115-124.

76. Guillon M. Are silicone hydrogel contact lenses more comfortable than hydrogel contact lenses? Eye Contact Lens. 2013;39:86-92.

77. Young G, Veys J, Pritchard N, Coleman S. A multi-centre study of lapsed contact lens wearers. Ophthalmic Physiol Opt. 2002;22:516-527.

78. Pritchard N, Fonn D, Brazeau D. Discontinuation of contact lens wear: a survey. Int Contact Lens Clin. 1999;26:157-162.

79. Dumbleton K, Woods CA, Jones LW, Fonn D. The impact of contemporary contact lenses on contact lens discontinuation. Eye Contact Lens. 2013;39:93-99.

80. Holden B, Sweeney D, Seger RG. Epithelial erosions caused by thin high water content lenses. Clin Exp Optom. 1986;69: 103-107.

81. du Toit R, Situ P, Simpson T, Fonn D. The effects of six months of contact lens wear on the tear film, ocular surfaces, and symptoms of presbyopes. Optom Vis Sci. 2001;78:455-462.

82. Begley CG, Chalmers RL, Abetz L, et al. The relationship between habitual patient-reported symptoms and clinical signs among patients with dry eye of varying severity. Invest Ophthalmol Vis Sci. 2003;44:4753-4761.

83. Opdahl A, Kim SH, Koffas TS, Marmo C, Somorjai GA. Surface mechanical properties of pHEMA contact lenses: viscoelastic and adhesive property changes on exposure to controlled humidity. J Biomed Mater Res A. 2003;67:350-356.

84. Pritchard N, Fonn D. Dehydration, lens movement and dryness ratings of hydrogel contact lenses. Ophthalmic Physiol Opt. 1995;15:281-286.

85. Efron N, Brennan N. A survey of wearers of low water content hydrogel contact lenses. Clin Exp Optom. 1988;71: 86-90.

86. McCarey BE, Wilson LA. pH, osmolarity and temperature effects on the water content of hydrogel contact lenses. Contact Intraocul Lens Med J. 1982;8:158-167.

87. Young G, Garofalo R, Peters S, Harmer O. The effect of temperature on soft contact lens modulus and diameter. Eye Contact Lens. 2011;37:337-341.

88. Young G. Exploring the relationship between materials and ocular health and comfort. Contact Lens Spect. 2007;22:3740.

89. Little S, Bruce A. Environmental influences on hydrogel lens dehydration and the postlens tear film. Int Contact Lens Clin. 1995;22:148-155.

90. Efron N, Brennan N, O'Brien K, Murphy P. Surface hydration of hydrogel contact lenses. Clin Exp Optom. 1986;69:219222. 
91. Little SA, Bruce AS. Role of the post-lens tear film in the mechanism of inferior arcuate staining with ultrathin hydrogel lenses. CLAO J. 1995;21:175-181.

92. Mirejovsky D, Patel AS, Young G. Water properties of hydrogel contact lens materials: a possible predictive model for corneal desiccation staining. Biomaterials. 1993;14: 1080-1088.

93. Helton DO, Watson LS. Hydrogel contact lens dehydration rates determined by thermogravimetric analysis. CLAO J. 1991;17:59-61.

94. Jones L, May C, Nazar L, Simpson T. In vitro evaluation of the dehydration characteristics of silicone hydrogel and conventional hydrogel contact lens materials. Cont Lens Anterior Eye. 2002;25:147-156.

95. Jones R, Jones L. In vitro bulk dehydration rates of hydrogel and silicone hydrogel daily disposable and frequent replacement contact lens materials. Optom Vis Sci. 2010;87:eabstract 105217.

96. Gonzalez-Meijome JM, Lopez-Alemany A, Almeida JB, Parafita MA, Refojo MF. Qualitative and quantitative characterization of the in vitro dehydration process of hydrogel contact lenses. J Biomed Mater Res B Appl Biomater. 2007;83:512526.

97. Andrasko G. The amount and time course of soft contact lens dehydration. J Am Optom Assoc. 1982;53:207.

98. Brennan NA, Lowe R, Efron N, Ungerer JL, Carney LG. Dehydration of hydrogel lenses during overnight wear. $A m \mathrm{~J}$ Optom Physiol Opt. 1987;64:534-539.

99. Efron N, Brennan NA, Bruce AS, Duldig DI, Russo NJ. Dehydration of hydrogel lenses under normal wearing conditions. CLAO J. 1987;13:152-156.

100. Efron N, Young G. Dehydration of hydrogel contact lenses in vitro and in vivo. Ophthalmic Physiol Opt. 1988;8:253-256.

101. Brennan NA, Lowe $\mathrm{R}$, Efron $\mathrm{N}$, Harris MG. In vivo dehydration of disposable (Acuvue) contact lenses. Optom Vis Sci. 1990;67:201-203.

102. Fonn D, Situ P, Simpson T. Hydrogel lens dehydration and subjective comfort and dryness ratings in symptomatic and asymptomatic contact lens wearers. Optom Vis Sci. 1999;76: 700-704.

103. Lemp MA, Caffery B, Lebow K, et al. Omafilcon A (Proclear) soft contact lenses in a dry eye population. CLAO J. 1999;25: 40-47.

104. Morgan PB, Efron N. In vivo dehydration of silicone hydrogel contact lenses. Eye Contact Lens. 2003;29:173-176.

105. Morgan PB, Efron N, Morgan SL, Little SA. Hydrogel contact lens dehydration in controlled environmental conditions. Eye Contact Lens. 2004;30:99-102.

106. Ramamoorthy P, Sinnott LT, Nichols JJ. Contact lens material characteristics associated with hydrogel lens dehydration. Ophthalmic Physiol Opt. 2010;30:160-166.

107. Maldonado-Codina C, Efron N. Impact of manufacturing technology and material composition on the clinical performance of hydrogel lenses. Optom Vis Sci. 2004;81:442-454.

108. Kim SH, Marmo C, Somorjai GA. Friction studies of hydrogel contact lenses using AFM: non-crosslinked polymers of low friction at the surface. Biomaterials. 2001;22: 3285-3294.

109. Kim SH, Opdahl A, Marmo C, Somorjai GA. AFM and SFG studies of pHEMA-based hydrogel contact lens surfaces in saline solution: adhesion, friction, and the presence of noncrosslinked polymer chains at the surface. Biomaterials. 2002;23:1657-1666.

110. Rennie AC, Dickrell PL, Sawyer WG. Friction coefficient of soft contact lenses: measurements and modeling. Tribol Lett. 2005;18:499-504.
111. Dunn AC, Cobb JA, Kantzios AN, et al. Friction coefficient measurement of hydrogel materials on living epithelial cells. Tribol Lett. 2008;30:13-19.

112. Dunn AC, Uruena JM, Huo YC, Perry SS, Angelini TE, Sawyer WG. Lubricity of surface hydrogel layers. Tribol Lett. 2013; 49:371-378.

113. Zhou B, Li Y, Randall NX, Li L. A study of the frictional properties of senofilcon-A contact lenses. J Mech Behav Biomed Mater. 2011;4:1336-1342.

114. Ngai V, Medley J, Jones L, Forrest J, Teichroeb J. Friction of contact lenses: Silicone hydrogel versus conventional hydrogel. Tribol Interface Eng Ser. 2005;48:371-379.

115. Roba M, Duncan EG, Hill GA, Spencer ND, Tosatti SGP. Friction measurements of contact lenses in their operating environment. Tribol Lett. 2011;44:378-397.

116. Brennan NA. Contact lens based correlates of soft lens wearing comfort. Optom Vis Sci. 2009;86:e-abstract 90957.

117. Coles CML, Brennan NA. Coefficient of friction and soft contact lens comfort. Optom Vis Sci. 2012;88:e-abstract 125603

118. Ross G, Nasso M, Franklin V, Lyndon F, Tighe B. Silicone hydrogels: trends in products and properties. Poster presented at: BCLA 29th Clinical Conference \& Exhibition; June 3-5, 2005; Brighton, UK. Available at: http://www. siliconehydrogels.org/posters/dec_05.asp. Accessed Aug 31, 2013.

119. Andrasko G. Andrasko corneal staining grid. The Staining Grid Center. Available at: www.stainingrid.com. Accessed August 31, 2013.

120. Johnson RE, Dettre RH. Wetting of low-energy surfaces. In: Berg JC, ed. Wettability (Surfactant Science Series Volume 49). New York, NY: CRC Press; 1993:1-73.

121. Thai LC, Tomlinson A, Doane MG. Effect of contact lens materials on tear physiology. Optom Vis Sci. 2004;81:194204.

122. Guillon M, Maissa C. Contact lens wear affects tear film evaporation. Eye Contact Lens. 2008;34:326-330.

123. Craig JP, Tomlinson A. Importance of the lipid layer in human tear film stability and evaporation. Optom Vis Sci. 1997;74:8-13.

124. Nichols JJ, Mitchell GL, King-Smith PE. Thinning rate of the precorneal and prelens tear films. Invest Ophthalmol Vis Sci. 2005;46:2353-2361.

125. Nichols JJ, King-Smith PE. The impact of hydrogel lens settling on the thickness of the tears and contact lens. Invest Ophthalmol Vis Sci. 2004;45:2549-2554.

126. Glasson MJ, Stapleton F, Keay L, Willcox MD. The effect of short term contact lens wear on the tear film and ocular surface characteristics of tolerant and intolerant wearers. Cont Lens Anterior Eye. 2006;29:41-47; quiz 49.

127. Sarver MD, Bowman L, Bauman E, Dimartino R, Lau D, Umeda W. Wettability of some gas permeable hard contact lenses. Int Contact Lens Clin. 1984;11:479-490.

128. Knick PD, Huff JW. Effects of temperature and conditioning on contact lens wetting angles. CLAO J. 1991;17:177-180.

129. Read ML, Morgan PB, Kelly JM, Maldonado-Codina C. Dynamic contact angle analysis of silicone hydrogel contact lenses. J Biomater Appl. 2011;26:85-99.

130. Read ML, Morgan PB, Maldonado-Codina C. Measurement errors related to contact angle analysis of hydrogel and silicone hydrogel contact lenses. J Biomed Mater Res B Appl Biomater. 2009;91:662-668.

131. Cheng L, Muller SJ, Radke CJ. Wettability of silicone-hydrogel contact lenses in the presence of tear-film components. Curr Eye Res. 2004;28:93-108.

132. Ketelson HA, Meadows DL, Stone RP. Dynamic wettability properties of a soft contact lens hydrogel. Colloids Surf B Biointerfaces. 2005;40:1-9. 
133. Lin MC, Svitova TF. Contact lenses wettability in vitro: effect of surface-active ingredients. Optom Vis Sci. 2010;87:440447.

134. Thai LC, Tomlinson A, Ridder WH. In vitro and in vivo effects of a lubricant in a contact lens solution. Ophthalmic Physiol Opt. 2002;22:319-329.

135. Maldonado-Codina C, Efron N. Dynamic wettability of pHEMA-based hydrogel contact lenses. Ophthalmic Physiol Opt. 2006;26:408-418.

136. Nichols JJ, Mitchell GL, King-Smith PE. The impact of contact lens care solutions on the thickness of the tear film and contact lens. Cornea. 2005;24:825-832.

137. Tonge S, Jones L, Goodall S, Tighe B. The ex vivo wettability of soft contact lenses. Curr Eye Res. 2001;23:51-59.

138. Maldonado-Codina C, Morgan PB, Schnider CM, Efron N. Short-term physiologic response in neophyte subjects fitted with hydrogel and silicone hydrogel contact lenses. Optom Vis Sci. 2004;81:911-921.

139. Brennan NA, Coles ML, Ang JH. An evaluation of siliconehydrogel lenses worn on a daily wear basis. Clin Exp Optom. 2006;89:18-25.

140. Mengher LS, Bron AJ, Tonge SR, Gilbert DJ. A non-invasive instrument for clinical assessment of the pre-corneal tear film stability. Curr Eye Res. 1985;4:1-7.

141. Madden RK, Paugh JR, Wang C. Comparative study of two non-invasive tear film stability techniques. Curr Eye Res. 1994;13:263-269.

142. Guillon M, Styles E, Guillon JP, Maïssa C. Preocular tear film characteristics of nonwearers and soft contact lens wearers. Optom Vis Sci. 1997;74:273-279.

143. Guillon JP. Non-invasive Tearscope-Plus routine for contact lens fitting. Cont Lens Anterior Eye. 1998;21(suppl 1):S31S40.

144. Patel S. Hydrogel lens water content and the stability of the prelens tear film. Optom Vis Sci. 1991;68:783-785.

145. Thai LC, Tomlinson A, Doane MG. Effect of contact lens materials on tear physiology. Optom Vis Sci. 2004;81:194204

146. Thibos LN, Hong X. Clinical applications of the ShackHartmann aberrometer. Optom Vis Sci. 1999;76:817-825.

147. Tyagi G, Alonso-Caneiro D, Collins M, Read S. Tear film surface quality with rigid and soft contact lenses. Eye Contact Lens. 2012;38:171-178

148. Shiobara M, Schnider CM, Back A, Holden BA. Guide to the clinical assessment of on-eye wettability of rigid gas permeable lenses. Optom Vis Sci. 1989;66:202-206.

149. Peterson RC, Wolffsohn JS, Nick J, Winterton L, Lally J. Clinical performance of daily disposable soft contact lenses using sustained release technology. Cont Lens Anterior Eye. 2006;29:127-134.

150. Keir N, Jones L. Wettability and silicone hydrogel lenses: a review. Eye Contact Lens. 2013;39:100-108.

151. Maissa C, Tonge S, Rebeix V, Guillon M, Tighe B. Surface properties of daily disposable contact lenses. Poster presented at: BCLA Conference; June 4-6, 1998; Brighton, UK.

152. Mahomed A, Ross G, Tighe B. Contact lenses and comfort enhancers: In vivo and in vitro release of soluble PVA. Poster presented at: BCLA Conference; June 3-6, 2004; Brighton UK.

153. Winterton LC, Lally JM, Sentell KB, Chapoy LL. The elution of poly (vinyl alcohol) from a contact lens: the realization of a time release moisturizing agent/artificial tear. $J$ Biomed Mater Res B Appl Biomater. 2007;80:424-432.

154. Wolffsohn JS, Hunt OA, Chowdhury A. Objective clinical performance of 'comfort-enhanced' daily disposable soft contact lenses. Cont Lens Anterior Eye. 2010;33:88-92.
155. Rasul N, Tighe B. Ophthalmic applications of hyaluronic acid. Poster presented at: BCLA Conference; May 31-June 3, 2007; Manchester, UK

156. Rah MJ. A review of hyaluronan and its ophthalmic applications. Optometry 2011;82:38-43

157. Ali M, Byrne ME. Controlled release of high molecular weight hyaluronic acid from molecularly imprinted hydrogel contact lenses. Pharm Res. 2009;26:714-726.

158. Weeks A, Morrison D, Alauzun JG, Brook MA, Jones L, Sheardown H. Photocrosslinkable hyaluronic acid as an internal wetting agent in model conventional and silicone hydrogel contact lenses. J Biomed Mater Res A. 2012;100: 1972-1982.

159. Weeks A, Subbaraman LN, Jones L, Sheardown H. Physical entrapment of hyaluronic Acid during synthesis results in extended release from model hydrogel and silicone hydrogel contact lens materials. Eye Contact Lens. 2013;39:179-185.

160. Fagnola M, Pagani MP, Maffioletti S, Tavazzi S, Papagni A. Hyaluronic acid in hydrophilic contact lenses: spectroscopic investigation of the content and release in solution. Cont Lens Anterior Eye. 2009;32:108-112.

161. Uccello-Barretta G, Nazzi S, Zambito Y, Di Colo G, Balzano F, Sansò M. Synergistic interaction between TS-polysaccharide and hyaluronic acid: implications in the formulation of eye drops. Int J Pharm. 2010;395:122-131.

162. Atkins N. Fusion 1-day: Improving long term CL comfort. Optician. 2013;6391:20-24.

163. Fonn D, Gauthier CA, Pritchard N. Patient preferences and comparative ocular responses to rigid and soft contact lenses. Optom Vis Sci. 1995;72:857-863.

164. Morgan PB, Maldonado-Codina C, Efron N. Comfort response to rigid and soft hyper-transmissible contact lenses used for continuous wear. Eye Contact Lens. 2003;29:(suppl 1):S127S130; discussion S143-144, S192-S194.

165. Maldonado-Codina C, Morgan PB, Efron N, Efron S. Comparative clinical performance of rigid versus soft hyper Dk contact lenses used for continuous wear. Optom Vis Sci. 2005;82:536-548

166. Tomlinson A, Bibby MM. Movement and rotation of soft contact lenses. Effect of fit and lens design. Am J Optom Pbysiol Opt. 1980;57:275-279.

167. Lowther GE, Tomlinson A. Critical base curve and diameter interval in the fitting of spherical soft contact lenses. $\mathrm{Am} \mathrm{J}$ Optom Physiol Opt. 1981;58:355-360.

168. Hernandez V, Tomlinson A. The effects of soft lens edge thickness. Cont Lens Forum. 1983;8:77-83.

169. Young G, Holden B, Cooke G. Influence of soft contact lens design on clinical performance. Optom Vis Sci. 1993;70:394403.

170. Martin DK, Holden BA. A new method for measuring the diameter of the in vivo human cornea. Am J Optom Physiol Opt. 1982;59:436-441.

171. Hall LA, Young G, Wolffsohn JS, Riley C. The influence of corneoscleral topography on soft contact lens fit. Invest Ophthalmol Vis Sci. 2011;52:6801-6806.

172. Hall L, Hunt C, Young G, Wolffsohn J. Factors affecting the corneoscleral topography. Invest Ophthalmol Vis Sci. 2013; 54:3691-3701.

173. Mertz G, Kissack B, Walter H, Sadue-Sokolow A, Simons M. A simplified approach to fitting spin-cast hydrogel contact lenses - Part 1. Int Contact Lens Clin. 1979;6:77-85.

174. Mertz G, Simons M, Kissack B, Walter H, Sadue-Sokolow A. A simplified approach to fitting spin-cast hydrogel contact lenses - Part 2. Int Contact Lens Clin. 1979;6:48-56.

175. Wake E, Tienda JB, Uyekawa PM, Mandell RB. Centration and coverage of hydrogel contact lenses. Am J Optom Physiol opt. 1981;58:302-308. 
176. McNamara NA, Polse KA, Brand RJ, Graham AD, Chan JS, McKenney CD. Tear mixing under a soft contact lens: effects of lens diameter. Am J Ophthalmol. 1999;127:659-665.

177. Young G. Daily disposable soft lens diameters. Optician. 2008;235:24-26.

178. Chauhan A, Radke CJ. Modeling the vertical motion of a soft contact lens. Curr Eye Res. 2001;22:102-108.

179. Hoekel JR, Maydew TO, Bassi CJ, Bennett ES, Henry VA. An evaluation of the $8.4 \mathrm{~mm}$ and the $8.8 \mathrm{~mm}$ base curve radii in the Ciba NewVue vs the Vistakon Acuvue. Int Contact Lens Clin. 1994;21:14-18.

180. Martin DK, Boulos J, Gan J, Gavriel K, Harvey P. A unifying parameter to describe the clinical mechanics of hydrogel contact lenses. Optom Vis Sci. 1989;66:87-91.

181. Roseman MJ, Frost A, Lawley ME. Effects of base curve on the fit of thin, mid-water contact lenses. Int Contact Lens Clin. 1993;20:95-101.

182. Dumbleton KA, Chalmers RL, McNally J, Bayer S, Fonn D. Effect of lens base curve on subjective comfort and assessment of fit with silicone hydrogel continuous wear contact lenses. Optom Vis Sci. 2002;79:633-637.

183. Young G, Schnider C, Hunt C, Efron S. Corneal topography and soft contact lens fit. Optom Vis Sci. 2010;87:358-366.

184. Santodomingo-Rubido J, Rubido-Crespo MJ. The clinical investigation of the base curve and comfort rate of a new prototype silicone hydrogel contact lens. Eye Contact Lens. 2008;34:146-50.

185. Hubner T, Tamm M, Sickenberger W. Edge profiles of hydrogel contact lenses and their effect on fitting and wearing characteristics. 2010;2:1-8. Available at: http:// www.woehlk.com.hr/pdf/woehlk-wissen-02-en.pdf. Accessed Jun 3, 2013.

186. Wang J, Jiao S, Ruggeri M, Shousha MA, Chen $Q$. In situ visualization of tears on contact lens using ultra high resolution optical coherence tomography. Eye Contact Lens. 2009;35:44-49.

187. Shen M, Cui L, Riley C, Wang MR, Wang J. Characterization of soft contact lens edge fitting using ultra-high resolution and ultra-long scan depth optical coherence tomography. Invest Ophthalmol Vis Sci. 2011;52:4091-4097.

188. Kaluzny BJ, Kaluzny JJ, Szkulmowska A, et al. Spectral optical coherence tomography: a new imaging technique in contact lens practice. Ophthalmic Physiol Opt. 2006;26:127-132.

189. Brennan NA, Efron N. Symptomatology of HEMA contact lens wear. Optom Vis Sci. 1989;66:834-838.

190. Tomlinson A, Schoessler J, Andrasko G. The effect of varying prism and truncation on the performance of soft contact lenses. Am J Optom Physiol Opt. 1980;57:714-720.

191. Cho P, Cheung SW, Charm J. Visual outcome of Soflens Daily Disposable and Soflens Daily Disposable for Astigmatism in subjects with low astigmatism. Clin Exp Optom. 2012;95:4347.

192. Papas E, Young G, Hearn K. Monovision vs soft diffractive bifocal contact lenses: A crossover study. Int Contact Lens Clin. 1990;17:181-187.

193. Richdale K, Mitchell GL, Zadnik K. Comparison of multifocal and monovision soft contact lens corrections in patients with low-astigmatic presbyopia. Optom Vis Sci. 2006;83:266-273.

194. Johnson T, Schnider C. Clinical performance and patient preferences for hydrogel versus RGP lenses: A crossover study. Int Contact Lens Clin. 1991;18:130-135.

195. Smart C. The edge forms of contact lenses. Contact Lens J. 1984;12:5-16.

196. Sengor T, Kurna SA, Aki S, Ozkurt Y. High Dk piggyback contact lens system for contact lens-intolerant keratoconus patients. Clin Ophthalmol. 2011;5:331-335.
197. O'Donnell C, Maldonado-Codina C. A hyper-Dk piggyback contact lens system for keratoconus. Eye Contact Lens. 2004; 30:44-48

198. Stone J. Corneal lenses with constant axial edge lift. Ophthalmic Optician. 1975;15:818-824.

199. Cornish R, Sulaiman S. Do thinner rigid gas permeable contact lenses provide superior initial comfort? Optom Vis Sci. 1996;73:139-143.

200. Mandell RB. Contact Lens Practice: Basic and Advanced. Springfield, IL: Charles C. Thomas; 1965:192-195.

201. Shanks KR. Subjective comparison of corneal lens edges. $\mathrm{BrJ}$ Physiol Opt. 1966;23:55-58.

202. Korb D, Exford J. A new concept in contact lens designparts I and II. J Am Optom Assoc. 1970;[Vol]:1032-1032.

203. La Hood D. Edge shape and comfort of rigid lenses. Am J Optom Physiol Opt. 1988;65:613-618.

204. van der Worp E. A Guide to Scleral Lens Fitting [monograph online]. Scleral Lens Education Society; 2010. Available at: http://commons.pacificu.edu/mono/4/. Accessed August 25, 2013.

205. Sorbara L, Mueller K. Effect of lens diameter on lens performance and initial comfort of two types of GP lenses for keratoconus: a pilot study. J Optom. 2011;4:22-29.

206. Visser ES, Visser R, van Lier HJ, Otten HM. Modern scleral lenses part II: patient satisfaction. Eye Contact Lens. 2007;33: 21-25.

207. Polse KA. Tear flow under hydrogel contact lenses. Invest Ophthalmol Vis Sci. 1979;18:409-413.

208. Little SA, Bruce AS. Postlens tear film morphology, lens movement and symptoms in hydrogel lens wearers. Ophthalmic Physiol Opt. 1994;14:65-69.

209. Brennan N, Coles M. Extended wear in perspective. Optom Vis Sci. 1997;74:609-623.

210. Robboy MW, Comstock TL, Kalsow CM. Contact lensassociated corneal infiltrates. Eye Contact Lens. 2003;29: 146-154.

211. Josephson J, Caffery B. Infiltrative keratitis in hydrogel lens wearers. Int Cont Lens Clin. 1979;6:47-70.

212. Stein RM, Clinch TE, Cohen EJ, Genvert GI, Arentsen JJ, Laibson PR. Infected vs sterile corneal infiltrates in contact lens wearers. Am J Ophthalmol. 1988;105:632-636.

213. Nilsson SE, Persson G. Low complication rate in extended wear of contact lenses. A prospective two-year study of nonmedical high water content lens wearers. Acta Ophthalmol (Copenh). 1986;64:88-92.

214. Zantos S. Management of corneal infiltrates in extended-wear contact lens patients. Int Contact Lens Clin. 1984;11:604610.

215. Paugh JR, Stapleton F, Keay L, Ho A. Tear exchange under hydrogel contact lenses: methodological considerations. Invest Ophthalmol Vis Sci. 2001;42:2813-2820.

216. Lin MC, Soliman GN, Lim VA, et al. Scalloped channels enhance tear mixing under hydrogel contact lenses. Optom Vis Sci. 2006;83:874-878.

217. Weidemann KE, Lakkis C. Clinical performance of microchannel contact lenses. Optom Vis Sci. 2005;82:498-504.

218. Ang J, Efron N. Comfort of fenestrated hydrogel lenses. Clin Exp Optom. 1987;70:117-120.

219. Knapp J, inventor; Schering Corp., assignee. Color-imparting contact lenses. US patent US 4582402 A. April 15, 1986.

220. Kunzler J, Friends G, Ammon D, McGee J, Gartley M, inventors; Bausch + Lomb, assignee. Lens with colored portion and coated surface. US patent 7,147,326. January 13, 2006

221. Steffen R, Barr J. Clear versus opaque soft contact lenses: initial comfort comparison. Int Contact Lens Clin. 1993;20: 184-186. 
222. Voetz SC, Collins MJ, Lingelbach B. Recovery of corneal topography and vision following opaque-tinted contact lens wear. Eye Contact Lens. 2004;30:111-117.

223. Spraul CW, Roth HJ, Gäckle H, Lang GE, Lang GK. Influence of special-effect contact lenses (Crazy Lenses) on visual function. CLAO J. 1998;24:29-32.

224. Insler MS, Hendricks C, George DM. Visual field constriction caused by colored contact lenses. Arch Ophthalmol. 1988; 106:1680-1682.

225. Gauthier C, Grant T, Holden BA. Clinical performance of two opaque tinted soft contact lenses. J Am Optom Assoc. 1992; 63:344-349.

226. Bucci Jr FA, Evans RE, Moody KJ, Tanner JB, Capozza RC, Klyce SD. The annular tinted contact lens syndrome: corneal topographic analysis of ring-shaped irregular astigmatism caused by annular tinted contact lenses. CLAO J. 1997;23: 161-167.

227. Rogers R, Broad R, MacCabee G, Williams S, inventors; Novartis AG, assignee. Contact lens with moulded inversion mark. European patent EP1158339 B1. May 23, 2001.

228. Wu J, Chehab K, Crowe C, et al., inventors; Johnson \& Johnson, assignee. Contact lenses bearing marks. US patent 6,203,156. March 31, 1998.

229. Roth H. Alterations of contact lenses. In: Thieme S, ed. Contact Lens Complications. Stuttgart, Germany: Thieme Medical Publishers.

230. Doughman DJ, Mobilia E, Drago D, Havener V, Gavin M. The nature of "spots" on soft lenses. Ann Ophthalmol. 1975;7: 345-348, 351-353.

231. Lowther GE, Hilbert JA. Deposits on hydrophilic lenses: differential appearance and clinical causes. Am J Optom Physiol Opt. 1975;52:687-692.

232. Rudko P, Proby J. A method for classifying and describing protein deposition on the hydrophilic lens. In: Allergan Report Series. Irvine, CA: Allergan Pharmaceuticals; 1974.

233. Minno GE, Eckel L, Groemminger S, Minno B, Wrzosek T. Quantitative analysis of protein deposits on hydrophilic soft contact lenses: I. Comparison to visual methods of analysis. II. Deposit variation among FDA lens material groups. Optom Vis Sci. 1991;68:865-872.

234. Myers RI, Larsen DW, Tsao M, et al. Quantity of protein deposited on hydrogel contact lenses and its relation to visible protein deposits. Optom Vis Sci. 1991;68:776-782.

235. Nilsson SE, Andersson L. Contact lens wear in dry environments. Acta Ophthalmol (Copenh). 1986;64:221225.

236. Nilsson SE, Lindh H. Hydrogel contact lens cleaning with or without multi-enzymes. A prospective study. Acta Opbthalmol (Copenh). 1988;66:15-18.

237. Gellatly KW, Brennan NA, Efron N. Visual decrement with deposit accumulation of HEMA contact lenses. Am J Optom Physiol Opt. 1988;65:937-941.

238. Nason RJ, Vogel H, Tarbell BJ, Yi FP, Mertz GW. A clinical evaluation of frequent replacement contact lenses on patients currently wearing premium reusable daily wear soft contact lenses. J Am Optom Assoc. 1993;64:188-195.

239. Bruce A, Golding T, Au S, Rowhani H. Mechanism of dryness in soft lens wear - role of BUT and deposits. Clin Exp Optom. 1995;78:168-175.

240. Nason RJ, Boshnick EL, Cannon WM, et al. Multisite comparison of contact lens modalities. Daily disposable wear vs. conventional daily wear in successful contact lens wearers. J Am Optom Assoc. 1994;65:774-780.

241. Pritchard N, Fonn D, Weed K. Ocular and subjective responses to frequent replacement of daily wear soft contact lenses. CLAO J. 1996;22:53-59.
242. Solomon OD, Freeman MI, Boshnick EL, et al. A 3-year prospective study of the clinical performance of daily disposable contact lenses compared with frequent replacement and conventional daily wear contact lenses. CLAO J. 1996;22:250-257.

243. Stern J, Wong R, Naduvilath TJ, Stretton S, Holden BA, Sweeney DF. Comparison of the performance of 6- or 30night extended wear schedules with silicone hydrogel lenses over 3 years. Optom Vis Sci. 2004;81:398-406.

244. Nichols JJ. Deposition rates and lens care influence on galyfilcon A silicone hydrogel lenses. Optom Vis Sci. 2006;83: 751-757.

245. Lin ST, Mandell RB, Leahy CD, Newell JO. Protein accumulation on disposable extended wear lenses. CLAO J. 1991;17: 44-50.

246. Lever O, Groemminger S, Allen M, Bornemann R, Dey D, Barna B. Evaluation of the relationship between total lens protein deposition and patient-rated comfort of hydrophilic (soft) contact lenses. Int Contact Lens Clin. 1995;22:5-13.

247. Jones L, Franklin V, Evans K, Sariri R, Tighe B. Spoilation and clinical performance of monthly vs. three monthly Group II disposable contact lenses. Optom Vis Sci. 1996;73:16-21.

248. Bruinsma GM, Rustema-Abbing M, de Vries J, et al. Influence of wear and overwear on surface properties of etafilcon A contact lenses and adhesion of Pseudomonas aeruginosa. Invest Ophthalmol Vis Sci. 2002;43:3646-3653.

249. Michaud L, Giasson CJ. Overwear of contact lenses: increased severity of clinical signs as a function of protein adsorption. Optom Vis Sci. 2002;79:184-192.

250. Caron P, St-Jacques J, Michaud L. Clinical discussion on the relative efficacy of 2 surfactant-containing lubricating agents in removing proteins during contact lens wear. Optometry. 2007;78:23-29.

251. Subbaraman LN, Glasier MA, Varikooty J, Srinivasan S, Jones L. Protein deposition and clinical symptoms in daily wear of etafilcon lenses. Optom Vis Sci. 2012;89:1450-1459.

252. Lebow K, Christensen B. Cleaning efficacy and patient comfort: a clinical comparison of two contact lens care systems. Int Contact Lens Clin. 1996;23:87-92.

253. Santos L, Rodrigues D, Lira M, et al. The influence of surface treatment on hydrophobicity, protein adsorption and microbial colonisation of silicone hydrogel contact lenses. Cont Lens Anterior Eye. 2007;30:183-188.

254. Subbaraman LN, Bayer S, Senchyna M, Sheardown H, Jones L. Rewetting drops containing surface active agents improve the clinical performance of silicone hydrogel contact lenses. Optom Vis Sci. 2006;83:143-151.

255. Zhao Z, Naduvilath T, Flanagan JL, et al. Contact lens deposits, adverse responses, and clinical ocular surface parameters. Optom Vis Sci. 2010;87:669-674.

256. Subbaraman LN, Jones L. Kinetics of lysozyme activity recovered from conventional and silicone hydrogel contact lens materials. J Biomater Sci Polym Ed. 2010;21:343-358.

257. Berry M, Pult H, Purslow C, Murphy PJ. Mucins and ocular signs in symptomatic and asymptomatic contact lens wear. Optom Vis Sci. 2008;85:E930-E938.

258. Berry M, Purslow C, Murphy PJ, Pult H. Contact lens materials, mucin fragmentation and relation to symptoms. Cornea. 2012;31:770-776.

259. Poggio EC, Abelson M. Complications and symptoms in disposable extended wear lenses compared with conventional soft daily wear and soft extended wear lenses. CLAOJ. 1993;19:31-39.

260. Nichols JJ, Mitchell GL, Zadnik K. Daily disposable vs. disposable extended wear: a contact lens clinical trial. Optom Vis Sci. 2000;77:637-647. 
261. Aakre BM, Ystenaes AE, Doughty MJ, Austrheim $\varnothing$, Westerfjell B, Lie MT. A 6-month follow-up of successful refits from daily disposable soft contact lenses to continuous wear of high-Dk silicone-hydrogel lenses. Ophthalmic Physiol Opt. 2004;24:130-141.

262. Fowler SA, Allansmith MR. Evolution of soft contact lens coatings. Arch Ophthalmol. 1980;98:95-99.

263. Hart DE, DePaolis M, Ratner BD, Mateo NB. Surface analysis of hydrogel contact lenses by ESCA. CLAO J. 1993;19:169173.

264. Leahy CD, Mandell RB, Lin ST. Initial in vivo tear protein deposition on individual hydrogel contact lenses. Optom Vis Sci. 1990;67:504-511.

265. Tripathi RC, Tripathi BJ, Ruben M. The pathology of soft contact lens spoilage. Ophthalmology. 1980;87:365-380.

266. Brennan NA, Coles M-LC. Deposits and symptomatology with soft contact lens wear. Int Contact Lens Clin. 2000;27:75100.

267. Donshik P, Weinstock FJ, Wechsler S, et al. Disposable hydrogel contact lenses for extended wear. CLAO J. 1988;14: 191-194.

268. Kaye DB, Hayashi MN, Schenkein JB. A disposable contact lens program: a preliminary report. CLAO J. 1988;14:33-37.

269. Frangie J, Schiller S, Hill LA. Understanding lens performance for wearers of monthly replacement contact lenses. Optom Today. 2008;48:39-42.

270. Boswall GJ, Ehlers WH, Luistro A, Worrall M, Donshik PC. A comparison of conventional and disposable extended wear contact lenses. CLAO J. 1993;19:158-165.

271. Poggio EC, Abelson MB. Complications and symptoms with disposable daily wear contact lenses and conventional soft daily wear contact lenses. CLAO J. 1993;19:95-102.

272. Snyder C, Hammack GG. Daily disposed hydrogel lenses-a comparison with biweekly replacement. J Am Optom Assoc. 1994;65:164-168.

273. Nilsson SEG, Söderqvist M. Clinical performance of a daily disposable contact lens a 3-month prospective study. J Brit Contact Lens Assoc. 1995;18:81-86.

274. Sulley A, Meyler J. A comparison of patient satisfaction between monthly and two-weekly replacement. Optician. 2001;222:20-26.

275. Malet F, Schnider CM. Influence of replacement schedule and care regimen on patient comfort and satisfaction with daily wear frequent-replacement contact lenses. CLAO J. 2002;28: 124-127.

276. Dumbleton K, Woods C, Jones L, Richter D, Fonn D. Comfort and vision with silicone hydrogel lenses: effect of compliance. Optom Vis Sci. 2010;87:421-425.

277. Lazon de la Jara P, Papas E, Diec J, Naduvilath T, Willcox MD, Holden BA. Effect of lens care systems on the clinical performance of a contact lens. Optom Vis Sci. 2013;90:344-350.

278. Jones L, Jones D, et al. The impact of replacement frequency and care regime on subjective satisfaction with disposable/ frequent replacement lenses. Optom Vis Sci. 1999;76(suppl 12): 172

279. Chalmers RL, Begley CG. Dryness symptoms among an unselected clinical population with and without contact lens wear. Cont Lens Anterior Eye. 2006;29:25-30.

280. Begley CG, Chalmers RL, Mitchell GL, et al. Characterization of ocular surface symptoms from optometric practices in North America. Cornea. 2001;20:610-618.

281. Nichols JJ, Ziegler C, Mitchell GL, Nichols KK. Self-reported dry eye disease across refractive modalities. Invest Ophthalmol Vis Sci. 2005;46:1911-1914.

282. Begley CG, Caffery B, Nichols KK, Chalmers R. Responses of contact lens wearers to a dry eye survey. Optom Vis Sci. 2000;77:40-46.
283. Coles ML, Brennan NA, Shuley V, et al. The influence of lens conditioning on signs and symptoms with new hydrogel contact lenses. Clin Exp Optom. 2004;87:367-371.

284. Guillon M, Maissa C. Use of silicone hydrogel material for daily wear. Cont Lens Anterior Eye. 2007;30:5-10.

285. Keir N, Woods CA, Dumbleton K, Jones L. Clinical performance of different care systems with silicone hydrogel contact lenses. Cont Lens Anterior Eye. 2010;33: 189-195.

286. Long B, McNally J. The clinical performance of a silicone hydrogel lens for daily wear in an Asian population. Eye Contact Lens. 2006;32:65-71.

287. Gonzalez-Meijome JM, Parafita MA, Yebra-Pimentel E, Almeida JB. Symptoms in a population of contact lens and noncontact lens wearers under different environmental conditions. Optom Vis Sci. 2007;84:296-302.

288. Bitton E, Jones L, Simpson T, Woods C. Influence of the blink interval on tear meniscus height in soft contact lens and nonlens wearers. Eye Contact Lens. 2010;36:156-163.

289. Martin R, Sanchez I, de la Rosa C, et al. Differences in the daily symptoms associated with the silicone hydrogel contact lens wear. Eye Contact Lens. 2010;36:49-53.

290. Diec J, Lazon de la Jara P, Willcox M, Holden BA. The clinical performance of lenses disposed of daily can vary considerably. Eye Contact Lens. 2012;38:313-318.

291. Gonzalez-Meijome JM, da Silva AC, Neves H, Lopes-Ferreira D, Queirós A, Jorge J. Clinical performance and "ex vivo" dehydration of silicone hydrogel contact lenses with two new multipurpose solutions. Cont Lens Anterior Eye. 2013; 36:86-92.

292. Walker J, Young G, Hunt C, Henderson T. Multi-centre evaluation of two daily disposable contact lenses. Cont Lens Anterior Eye. 2007;30:125-133.

293. Stiegemeier MJ, Friederichs GJ, Hughes JL, Larsen S, Movic W, Potter WB. Clinical evaluation of a new multi-purpose disinfecting solution in symptomatic contact lens wearers. Cont Lens Anterior Eye. 2006;29:143-151.

294. Richdale K, Sinnott LT, Skadahl E, Nichols JJ. Frequency of and factors associated with contact lens dissatisfaction and discontinuation. Cornea. 2007;26:168-174.

295. Korb DR, Greiner JV, Herman JP, et al. Lid-wiper epitheliopathy and dry-eye symptoms in contact lens wearers. CLAOJ. 2002;28:211-216.

296. Papas E, Golebiowski B, Tomlinson D, Williams J, Chan E, Chan J. Effect of daytime lens replacement on soft contact lens related discomfort. Cont Lens Anterior Eye. 2007;30: 302-303.

297. Chalmers R. A review of the metabolism of hydrogen peroxide by external ocular structures. Int Contact Lens Clin. 1995;22:143-146.

298. Chalmers R, McNally J. Ocular detection threshold for hydrogen peroxide: drops vs. lenses. Int Contact Lens Clin. 1988; 15:351-357.

299. Paugh JR, Brennan NA, Efron N. Ocular response to hydrogen peroxide. Am J Optom Physiol Opt. 1988;65:91-98.

300. Riley M, Wilson G. Topical hydrogen peroxide and the safety of ocular tissues. CLAO J. 1993;19:186-190.

301. Begley C, Edrington T, Chalmers R. Effect of lens care systems on corneal fluorescein staining and subjective comfort in hydrogel lens wearers. Int Contact Lens Clin. 1994;21:7-12.

302. Stiegemeier MJ, Cedrone R, Evans D, et al. Clinical performance of 'no rub' multi-purpose solutions. Cont Lens Anterior Eye. 2004;27:65-74.

303. Epstein AB. Contact lens care products effect on corneal sensitivity and patient comfort. Eye Contact Lens. 2006;32: 128-132. 
304. Morgan PB. Clinical investigation of two contact lens care products and their relationship to corneal sensitivity, comfort and corneal staining. Eye Contact Lens. 2007;33:54-55.

305. Santodomingo-Rubido J. The comparative clinical performance of a new polyhexamethylene biguanide- vs a polyquad-based contact lens care regime with two silicone hydrogel contact lenses. Ophthalmic Physiol Opt. 2007;27: 168-173.

306. Lipener C; Contact Lens Advisory in Scientific Studies Group. A randomized clinical comparison of OPTI-FREE EXPRESS and ReNu MultiPLUS multipurpose lens care solutions. Adv Ther. 2009;26:435-446.

307. Sorbara L, Peterson R, Woods C, Fonn D. Multipurpose disinfecting solutions and their interactions with a silicone hydrogel lens. Eye Contact Lens. 2009;35:92-97.

308. Young G, Keir N, Hunt C. Clinical evaluation of long-term users of two contact lens care preservative systems. Eye Contact Lens. 2009;35:50-58.

309. Corbin GS, Bennett L, Hunt C. A multicenter investigation of OPTI-FREE RepleniSH multi-purpose disinfecting solution impact on soft contact lens patient comfort. Clin Ophthalmol. 2010;4:47-57.

310. Huo Y, Ketelson H, Perry SS. Ethylene oxide-block-butylene oxide copolymer uptake by silicone hydrogel contact lens materials. Appl Surf Sci. 2013;273:472-477.

311. Weikart CM, Miyama M, Yasuda HK. Surface modification of conventional polymers by depositing plasma polymers of Trimethylsilane and of Trimethylsilane + O2. II. Dynamic wetting properties. J Colloid Interface Sci. 1999;211:28-38.

312. Maldonado-Codina $C$, Morgan PB. In vitro water wettability of silicone hydrogel contact lenses determined using the sessile drop and captive bubble techniques. J Biomed Mater Res A. 2007;83:496-502.

313. Bruinsma GM, van der Mei HC, Busscher HJ. Bacterial adhesion to surface hydrophilic and hydrophobic contact lenses. Biomaterials. 2001;22:3217-3224.

314. Jones L, Senchyna M. Soft contact lens solutions review: part 1 - components of modern care regimens. Optom Practice. 2007;8:45-56.

315. Jones L, Christie C. Soft contact lens solutions review: part 2 modern generation care systems. Optom Practice. 2008;9: 43-62.

316. Simmons PA, Donshik PC, Kelly WF, Vehige JG. Conditioning of hydrogel lenses by a multipurpose solution containing an ocular lubricant. CLAO J. 2001;27:192-194.

317. Toda I, Shinozaki N, Tsubota K. Hydroxypropyl methylcellulose for the treatment of severe dry eye associated with Sjogren's syndrome. Cornea. 1996;15:120-128.

318. Yang SN, Tai YC, Sheedy JE, Kinoshita B, Lampa M, Kern JR. Comparative effect of lens care solutions on blink rate, ocular discomfort and visual performance. Ophthalmic Physiol Opt. 2012;32:412-420.

319. Campbell R, Kame G, Leach N, Paul M, White E, Zigler L. Clinical benefits of a new multipurpose disinfecting solution in silicone hydrogel and soft contact lens users. Eye Contact Lens. 2012;38:93-101.

320. Christensen B, Lebow K, White EM, Cedrone R, Bevington R. Effectiveness of citrate-containing lens care regimens: a controlled clinical comparison. Int Contact Lens Clin. 1998;25:50-58.

321. Guillon M, Maissa C. Clinical acceptance of two multipurpose solutions: MPS containing HPMC versus citrate-based MPS without rubbing. CLAO J. 2002;28:186-191.

322. Jones L, Senchyna M, Glasier MA, et al. Lysozyme and lipid deposition on silicone hydrogel contact lens materials. Eye Contact Lens. 2003;29:(suppl 1):S75-S79; discussion S83S84, S192-S194.
323. Powell CH, Lally JM, Hoong LD, Huth SW. Lipophilic versus hydrodynamic modes of uptake and release by contact lenses of active entities used in multipurpose solutions. Cont Lens Anterior Eye. 2010;33:9-18.

324. Jones L, Powell $\mathrm{CH}$. Uptake and release phenomena in contact lens care by silicone hydrogel lenses. Eye Contact Lens. 2013;39:29-36.

325. Rosenthal RA, Dassanayake NL, Schlitzer RL, Schlech BA, Meadows DL, Stone RP. Biocide uptake in contact lenses and loss of fungicidal activity during storage of contact lenses. Eye Contact Lens. 2006;32:262-266.

326. Willcox MD, Phillips B, Ozkan J, et al. Interactions of lens care with silicone hydrogel lenses and effect on comfort. Optom Vis Sci. 2010;87:839-846.

327. Green JA, Phillips KS, Hitchins VM, et al. Material properties that predict preservative uptake for silicone hydrogel contact lenses. Eye Contact Lens. 2012;38:350-357.

328. Jones L, MacDougall N, Sorbara LG. Asymptomatic corneal staining associated with the use of balafilcon siliconehydrogel contact lenses disinfected with a polyaminopropyl biguanide-preserved care regimen. Optom Vis Sci. 2002;79: 753-761.

329. Andrasko G, Ryen K. Corneal staining and comfort observed with traditional and silicone hydrogel lenses and multipurpose solution combinations. Optometry. 2008;79:444-454.

330. Carnt NA, Evans VE, Naduvilath TJ, et al. Contact lens-related adverse events and the silicone hydrogel lenses and daily wear care system used. Arch Ophthalmol. 2009;127:1616-1623.

331. Garofalo RJ, Dassanayake N, Carey C, Stein J, Stone R, David R. Corneal staining and subjective symptoms with multipurpose solutions as a function of time. Eye Contact Lens. 2005; 31:166-174.

332. Diec J, Evans VE, Tilia D, Naduvilath T, Holden BA, Lazon de la Jara P. Comparison of ocular comfort, vision, and SICS during silicone hydrogel contact lens daily wear. Eye Contact Lens. 2012;38:2-6.

333. Tilia D, Lazon de la Jara P, Peng N, Papas EB, Holden BA. Effect of lens and solution choice on the comfort of contact lens wearers. Optom Vis Sci. 2013;90:411-418.

334. Carney LG, Hill RM. Human tear pH. Diurnal variations. Arch Ophthalmol. 1976;94:821-824.

335. Carney LG, Fullard RJ. Ocular irritation and environmental pH. Aust J Optom. 1979;62:335-336.

336. Tang I, Wong DM, Yee DJ, Harris MG. The $\mathrm{pH}$ of multipurpose soft contact lens solutions. Optom Vis Sci. 1996;73: 746-749.

337. Harris MG, Higa CK, Lacey LL, Barnhart LA. The pH of aerosol saline solution. Optom Vis Sci. 1990;67:84-88.

338. Harris MG, Torres J, Tracewell L. pH and $\mathrm{H} 2 \mathrm{O} 2$ concentration of hydrogen peroxide disinfection systems. Am J Optom Physiol Opt. 1988;65:527-535.

339. Dalton K, Subbaraman LN, Rogers R, Jones L. Physical properties of soft contact lens solutions. Optom Vis Sci. 2008;85:122-128.

340. Pandit JC, Nagyova B, Bron AJ, Tiffany JM. Physical properties of stimulated and unstimulated tears. Exp Eye Res. 1999;68: 247-253.

341. Tiffany JM. The viscosity of human tears. Int Opbthalmol. 1991;15:371-376.

342. Donshik P, Madden R, Simmons PA. Pursuing comfort in a multipurpose solution. Contact Lens Spect. 2000;1:33-36.

343. Tomlinson A, Khanal S. Assessment of tear film dynamics: quantification approach. Ocul Surf. 2005;3:81-95.

344. Farris RL, Gilbard JP, Stuchell RN, Mandel ID. Diagnostic tests in keratoconjunctivitis sicca. CLAO J. 1983;9:23-28. 
345. Gilbard JP, Farris RL, Santamaria II J. Osmolarity of tear microvolumes in keratoconjunctivitis sicca. Arch Ophthalmol. 1978;96:677-681.

346. Farris RL. Tear osmolarity-a new gold standard? Adv Exp Med Biol. 1994;350:495-503.

347. Tiffany JM, Pandit JC, Bron AJ. Soluble mucin and the physical properties of tears. Adv Exp Med Biol. 1998;438: 229-234.

348. Calvao-Santos G, Borges C, Nunes S, Salgado-Borges J, Duarte L. Efficacy of 3 different artificial tears for the treatment of dry eye in frequent computer users and/or contact lens users. Eur J Ophthalmol. 2011;21:538-544.

349. Guillon M, Maissa C, Pouliquen P, Delval L. Effect of povidone $2 \%$ preservative-free eyedrops on contact lens wearers with computer visual syndrome: pilot study. Eye Contact Lens. 2004;30:34-39.

350. Caffery BE, Josephson JE. Is there a better "comfort drop"? J Am Optom Assoc. 1990;61:178-182.

351. Golding TR, Efron N, Brennan NA. Soft lens lubricants and prelens tear film stability. Optom Vis Sci. 1990;67:461465 .
352. Efron N, Golding TR, Brennan NA. The effect of soft lens lubricants on symptoms and lens dehydration. CLAO J. 1991; 17:114-119.

353. Malet F, Karsenti D, Pouliquen P. Preservative-free ocular hydrating agents in symptomatic contact lens wearers: saline versus PVP solution. Eye Contact Lens. 2003;29:38-43.

354. Salpekar A, Tonge S, inventors; Bausch + Lomb, assignee. Contact lens packing solutions. US patent $6,440,366$. August 27, 2002.

355. Pruitt J, Lindley K, Winterton L. Triple-action moisturisers for increased comfort in daily disposable lenses. Optician. 2007; 234:27-28.

356. Menzies $\mathrm{KL}$, Rogers $\mathrm{R}$, Jones $\mathrm{L}$. In vitro contact angle analysis and physical properties of blister pack solutions of daily disposable contact lenses. Eye Contact Lens. 2010;36: 10-18.

357. Menzies KL, Jones L. In vitro analysis of the physical properties of contact lens blister pack solutions. Optom Vis Sci. 2011;88:493-501.

358. Pence NA. Thinking inside the blister. Contact Lens Spect. 2009;24:24.

359. Mack CJ. A new daily disposable design provides opportunity. Contact Lens Spect. 2009;24:48-49.

Appendix A. Examples of Some Commonly Prescribed Hydrogel Contact Lens Materials

\begin{tabular}{|c|c|c|c|c|}
\hline Commercial Name & Manufacturer & Water Content & $\mathbf{C T}$ & $\mathbf{D k} / \mathbf{t}$ \\
\hline SofLens 38 (polymacon) & Bausch + Lomb & 38 & 0.035 & 22 \\
\hline Biomedics 55 (ocufilcon D) & CooperVision & 55 & 0.07 & 21 \\
\hline Acuvue 2 (etafilcon $\mathrm{A})$ & Johnson \& Johnson & 58 & 0.084 & 20 \\
\hline SofLens daily disposable (hilafilcon B) & Bausch + Lomb & 59 & 0.09 & 19 \\
\hline PROCLEAR (omafilcon A) & CooperVision & 62 & 0.065 & 30 \\
\hline Focus Dailies (nelfilcon A) & CIBA Vision & 69 & 0.10 & 26 \\
\hline
\end{tabular}

Appendix B. Examples of Some Commonly Prescribed Silicone Hydrogel Contact Lens Materials

\begin{tabular}{|c|c|c|c|c|c|c|}
\hline Commercial Name & Manufacturer & $\begin{array}{c}\text { Water } \\
\text { Content }\end{array}$ & $\mathbf{C T}$ & $\mathbf{D k} / \mathbf{t}$ & $\begin{array}{l}\text { Modulus, } \\
\text { MPa }\end{array}$ & Surface Treatment \\
\hline Air Optix Night \& Day Aqua (lotrafilcon A) & Alcon & 24 & 0.08 & 175 & 1.4 & 25-nm plasma coating \\
\hline DAILIES TOTAL1 (delefilcon A) & Alcon & 33 & 0.09 & 156 & 0.7 & Soft surface gel with $>80 \%$ water content \\
\hline PureVision (balafilcon A) & Bausch + Lomb & 36 & 0.09 & 101 & 1.1 & Plasma oxidation process \\
\hline Acuvue OASYS (senofilcon A) & Johnson \& Johnson & 38 & 0.07 & 147 & 0.72 & None; internal wetting agent (PVP) \\
\hline Acuvue Advance (galyfilcon A) & Johnson \& Johnson & 47 & 0.07 & 86 & 0.40 & None; internal wetting agent (PVP) \\
\hline AVAIRA (enfilcon A) & CooperVision & 46 & 0.08 & 125 & 0.50 & None \\
\hline Menicon PremiO (asmofilcon A) & Menicon & 40 & 0.08 & 161 & 0.90 & Plasma oxidation \\
\hline Clariti (Filcon II 3) & Sauflon & 58 & 0.07 & 86 & 0.50 & Nondisclosed \\
\hline Definitive (efrofilcon A) & Contamac & 75 & $0.08^{*}$ & 76 & 0.35 & None \\
\hline
\end{tabular}

PVP, polyvinyl pyrrolidone; USAN, United States Adopted Name.

* Estimated as lathe-cut lens designed to practitioner specification. 